Aus der Klinik für Hals-Nasen-Ohrenheilkunde

(Prof. Dr. med. M. Canis)

im Zentrum Augenheilkunde

und Hals-, Nasen-, Ohrenheilkunde

der Medizinischen Fakultät der Universität Göttingen

\title{
Analyse des Notfallaufkommens an der HNO-Klinik der Universitätsmedizin \\ Göttingen im Jahr 2011
}

\author{
Inaugural-Dissertation \\ zur Erlangung des Doktorgrades der Zahnheilkunde \\ der Medizinischen Fakultät der \\ Georg-August-Universität zu Göttingen
}

vorgelegt von

Anna Lena Petersen

aus

Eutin

Göttingen 2015 
Dekan:

I. Berichterstatter:

II. Berichterstatter/in:
Prof. Dr. rer. nat. H. K. Kroemer

Prof. Dr. med. R. Laskawi

Prof. Dr. med Dr. PH. Martin Bauer

Tag der mündlichen Prüfung: 28.09.2015 


\section{Inhaltsverzeichnis}

\begin{tabular}{lll}
\hline 1 & Einleitung & 1
\end{tabular}

1.1 Notfalldefinitionen . . . . . . . . . . . . . . . . 2

1.2 Notdienstregelung . . . . . . . . . . . . . . . . . . . . . 3

1.2 .1 Zuständigkeiten für Notfälle . . . . . . . . . . . . . . . 3

1.2.2 Notdienstregelung für niedergelassene Ärzte in Niedersachsen ....................... 3

1.2 .3 Notdienstregelung in Göttingen . . . . . . . . . . . . 4

1.2.4 Regionale Abweichungen der Notdienstregelungen . . . . 5

1.2.5 HNO-Notdienst in der Universitätsmedizin Göttingen . . 6

1.3 Motivation und Zielsetzung der Arbeit . . . . . . . . . . . . 6

1.4 Übersicht der Arbeit . . . . . . . . . . . . . . . . . . . 8

2 Material und Methoden 9

$2.1 \quad$ Mittelfristige Entwicklung der Patientenzahlen . . . . . . . . . . 9

2.2 Einschlusskriterien . . . . . . . . . . . . . . . . . . . . . . 9

2.3 Datenquellen und Datentabelle . . . . . . . . . . . . . . . . 9

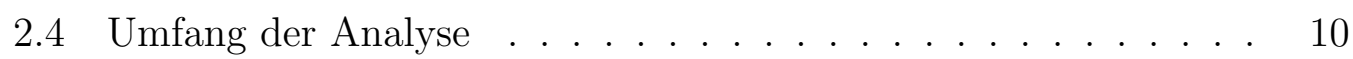

2.5 Aufbau der Analyse . . . . . . . . . . . . . . . . . . . . . 10

2.6 Generelle Patientendaten . . . . . . . . . . . . . . . . . . . . . 11

2.6 .1 Aufnahmeart . . . . . . . . . . . . . . 11

2.6 .2 Diagnosen . . . . . . . . . . . . . . . . . . . . . . . 11

$2.6 .3 \quad$ Altersstruktur . . . . . . . . . . . . . . . . . . . 11

2.6 .4 Einzugsgebiet . . . . . . . . . . . . . . . . . . 12

2.6 .5 Stoßzeiten . . . . . . . . . . . . . . . . . . . 12 
2.7 Spezielle Patientendaten . . . . . . . . . . . . . . . . . . 13

2.7 .1 Risikofaktoren . . . . . . . . . . . . . . . . . 13

2.7.2 Anzahl der Operationen der häufigsten Erkrankungen . . 14

2.7 .3 Besonderheiten der Diagnose „Nachblutungen ${ }^{4}$. . . . . . 14

2.7 .4 Konsile. . . . . . . . . . . . . . . . . . . 14

2.7 .5 Nachsorge . . . . . . . . . . . . . . . . 15

2.8 Statistische Auswertung $\ldots \ldots \ldots$. . . . . . . . . . . . . 15

\begin{tabular}{lll}
\hline 3 & Ergebnisse & 17
\end{tabular}

$3.1 \quad$ Mittelfristige Entwicklung der Patientenzahlen und Umfang der Analyse . . . . . . . . . . . . . . . . . . . 17

3.2 Generelle Patientendaten . . . . . . . . . . . . . . . . . . . . 18

3.2 .1 Aufnahmeart . . . . . . . . . . . . . . . . . . . . 18

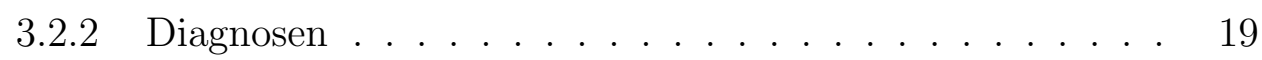

$3.2 .3 \quad$ Altersstruktur . . . . . . . . . . . . . . . . . . . . . 21

3.2 .4 Einzugsgebiet . . . . . . . . . . . . . . . . . 23

3.2 .5 Stoßzeiten . . . . . . . . . . . . . . . . . . . . . . . 27

3.3 Spezielle Patientendaten . . . . . . . . . . . . . . . . . . . . . 30

3.3 .1 Risikofaktor Alter . . . . . . . . . . . . . . . . 30

3.3.2 Risikofaktoren Allgemeinerkrankungen und Antikoagulanzien am Beispiel Epistaxis . . . . . . . . . . . . . . . . . 32

3.3.3 Anzahl der Operationen bei den häufigsten Erkrankungen 36

3.3 .4 Besonderheiten der Diagnose „Nachblutung ${ }^{6}$. . . . . . . 37

3.3 .5 Konsile . . . . . . . . . . . . . . . . . . . . . . . . . . . . . 38

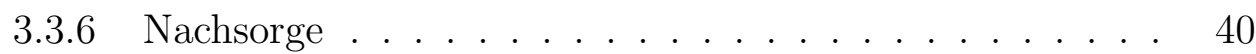

4 Diskussion 43

4.1 Überblick . . . . . . . . . . . . . . . . . . . . . . . . . . . . . . . 43

4.2 Methodik . . . . . . . . . . . . . . . . . . . . . . . 43

4.2 .1 Retrospektive Analyse . . . . . . . . . . . . . . 43

$4.2 .2 \quad$ Deskriptive und induktive Statistik . . . . . . . . . . . . 44 
4.3 Mittelfristige Entwicklung der Patientenzahlen und Umfang der Analyse . . . . . . . . . . . . . . . . 45

4.4 Generelle Patientendaten . . . . . . . . . . . . . . . . . . . . . . 47

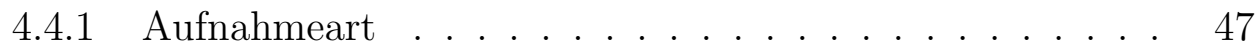

4.4 .2 Diagnosen . . . . . . . . . . . . . . . . . . . . . 47

4.4 .3 Altersstruktur . . . . . . . . . . . . . . . . . . . . . 51

4.4 .4 Einzugsgebiet . . . . . . . . . . . . . . . . 52

4.4 .5 Stoßzeiten . . . . . . . . . . . . . . . . 54

4.5 Spezielle Patientendaten . . . . . . . . . . . . . . . . 57

4.5 .1 Risikofaktor Alter . . . . . . . . . . . . . . 57

4.5.2 Risikofaktoren Allgemeinerkrankungen und Antikoagulanzien bei Epistaxis-Patienten . . . . . . . . . . . . . . 58

4.5.3 Anzahl der Operationen unter den häufigsten Diagnosen 59

4.5 .4 Besonderheiten der Diagnose „Nachblutung ${ }^{6}$. . . . . . . 59

4.5 .5 Konsile. . . . . . . . . . . . . . . . . . 61

4.5 .6 Nachsorge . . . . . . . . . . . . . . . . . . 62

4.6 Schlussfolgerungen . . . . . . . . . . . . . . . . . . 63

4.6 .1 Regulierung der Patientenzahl . . . . . . . . . . . . 63

4.6 .2 Steigerung der Qualität. . . . . . . . . . . . . . . 64

4.7 Ausblick . . . . . . . . . . . . . . . . . . . . . . . . . 65

$\begin{array}{lll}5 & \text { Zusammenfassung } & 66\end{array}$

\begin{tabular}{lll}
\hline 6 & Anhang & 68
\end{tabular}

6.1 Abbildungsverzeichnis . . . . . . . . . . . . . . . . . 75

6.2 Tabellenverzeichnis . . . . . . . . . . . . . . . . . . . . 79

6.3 Literaturverzeichnis . . . . . . . . . . . . . . . . . . . . 80 


\section{Abkürzungsverzeichnis}

Abb. Abbildung

abs. absolut

ASS Acetylsalicylsäure

BO Bereitschaftsdienstordnung

BUKH Abteilung des Berufsgenossenschaftlichen Unfallkrankenhauses

COPD chronische obstruktive Lungenerkrankung

Gr. Gruppe

HKE Herz-Kreislauf-Erkrankung

HNO Hals-Nasen-Ohrenheilkunde

ICD-10 Internationale statistische Klassifikation der Krankheiten und verwandter Gesundheitsprobleme

KVN Kassenärztliche Vereinigung Niedersachen

MKG Mund-, Kiefer- und Gesichtschirurgie

rel. relativ

SGB Sozialgesetzbuch

s. o. siehe oben

THG Thorax-, Herz- und Gefäßchirurgie

UMG Universitätsmedizin Göttingen 


\section{Einleitung}

Der Notdienst der Hals-Nasen-Ohrenheilkunde (HNO) der Universitätsmedizin Göttingen (UMG) übernimmt eine wichtige Funktion in der regionalen Patientenversorgung. Er gewährleistet durch die Regelungen der Kassenärztlichen Vereinigung Niedersachsen (KVN) eine lückenlose Versorgung auch außerhalb der üblichen Sprechzeiten und ist auf die Behandlung schwerwiegender Fälle spezialisiert.

Im Jahr 2003 wurde jedoch ein Fallpauschalen-System im Gesundheitswesen eingeführt, das die Krankenhäuser fortan zu Kosteneinsparungen und Strukturreformen gezwungen hat. Das neue Entgeltsystem wird als „größte Strukturreform im Krankenhausbereich seit über 30 Jahren“ bezeichnet (Blum und Offermanns, 2009, S. 295). Es führe dazu, dass sich Krankenhäuser strategisch neu positionierten, ihr Leistungsangebot überarbeiteten und Aufbau- und Ablauforganisationen neu strukturiert würden (Blum und Offermanns, 2009). Die Umsetzung dieser Reformen stellt auch den HNO-Notdienst vor besondere Herausforderungen, denn die Arbeit im Notdienst ist wenig planbar. Auf der einen Seite existieren Stoßzeiten mit hohem Patientenandrang, auf der anderen Seite gibt es Zeiten, in denen nur wenige Patienten den Notdienst aufsuchen. Verschärfend kommt hinzu, dass die Anzahl der Patienten seit mehreren Jahren gestiegen und ein Ende dieses Trends noch nicht absehbar ist. In der Literatur werden vor allem drei Gründe für diesen Andrang genannt:

1. Die Zahlen älterer Patienten steigen durch den demographischen Wandel. Sie benötigen häufiger umfangreiche medizinische Betreuung, da sie vermehrt unter chronischen Krankheiten leiden und der Anteil multimorbider Patienten höher ist (Morrmann, 2007; Hijano et al., 2009). 2. Patienten meinen nach 
ihrer Einschätzung nicht mehr bis zum nächsten Tag warten zu können, wenn sie außerhalb der Sprechzeiten krank werden (Hijano et al., 2009). 3. Viele Patienten kommen mit Diagnosen in die Notdienste, die nicht akut oder dringend behandlungsbedürftig sind (Gallo et al., 2000; Lasisi et al., 2007; Rivero et al., 2005; Schlicht und Guntinas-Lichius, 2012; Wheatley et al., 1999).

Es scheint also ein verbreitetes Phänomen zu sein, dass der Notdienst viele Behandlungen durchführt, für die er eigentlich nicht zuständig ist. Welche Gründe gibt es dafür, dass viele Patienten den Notdienst aufsuchen, aber nicht dringend behandlungsbedürftig sind? Eine mögliche Erklärung könnte sein, dass den Patienten die Zuständigkeiten des Notdienstes nicht vertraut sind. Daher ist es zunächst wichtig zu klären, was in dieser Arbeit als „Notfall“ angesehen wird. Ferner wird die rechtliche Ausgestaltung des Notdienstes in Niedersachsen und Göttingen beschrieben.

\subsection{Notfalldefinitionen}

Nach der Definition des medizinischen Lexikons „Pschyrembel“ wird von einem Notfall als einem „akuten, lebensbedrohlichen Zustand durch Störung der Vitalfunktionen oder Gefahr plötzlich eintretender, irreversibler Organschädigung ..." gesprochen (Pschyrembel, 2002, S. 1189). Fischer beschreibt den Notfall als ein „nicht geplantes, nicht vorhergesehenes Ereignis, das eine medizinische Behandlung notwendig macht"(Fischer, 2009, S. 37).

Wie schon diese Beispiele zeigen, gibt es in der Literatur keine einheitliche Definition des Begriffes „Notfall“. Der Kontext, in dem der Notfallbegriff verwendet wird, ist für die Definition maßgeblich (Killinger, 2009). So kann beispielsweise auch zwischen einem Notfall im Rahmen des Rettungsdienstes und einem Notfall im kassenärztlichen Notdienst differenziert werden.

Für die hier vorliegende Arbeit ist ausschließlich der Aufnahmezeitpunkt entscheidend. Kommt ein Patient nach Schließung der HNO-Poliklinik um 16:00 Uhr bis 7:30 Uhr am Folgetag oder aber am Wochenende in die HNO-Klinik, wird er hier als „Notfall“ angesehen. Die Definition dieser Arbeit richtet sich 
damit nach den tatsächlichen Gegebenheiten des HNO-Notdienstes, was nicht zwangsläufig mit einer rein medizinischen Definition eines "Notfalles“ übereinstimmen muss.

\subsection{Notdienstregelung}

\subsubsection{Zuständigkeiten für Notfälle}

Wer in Deutschland für die Behandlung von präklinischen Notfall-Patienten zuständig ist, entscheidet sich nach der Schwere des Notfalles. Lebensbedrohliche Fälle werden zunächst durch Notärzte im Rettungsdienst versorgt. Niedergelassene Ärzte, die sich außerhalb der Sprechzeiten in „Ärztlichen Notdiensten“ (auch Bereitschaftsdienst genannt) organisieren, betreuen hingegen meistens die nicht lebensbedrohlichen und nicht zeitkritischen Notfälle (Knuth, 1998). Daneben haben Patienten die Möglichkeit, die Notfallambulanz eines Krankenhauses aufzusuchen.

\subsubsection{Notdienstregelung für niedergelassene Ärzte in Niedersachsen}

Die Organisation und Regelung des Bereitschafts-, Rettungs- und Notdienstes ist je nach Bundesland unterschiedlich (Busse et al., 2013). Nach $\S 115$ Sozialgesetzbuch (SGB) V werden die Notdienste durch Verträge zwischen Krankenkassen, Krankenhäusern und Vertragsärzten geregelt. Es verhandeln jeweils die Vertretungen auf Landesebene miteinander.

Die niedergelassenen Ärzte in Niedersachsen werden über die KVN vertreten, der nach $\S 75$ Abs. 1 SGB V im Rahmen des Sicherstellungsauftrags die vertragsärztliche Versorgung außerhalb der Sprechzeiten übertragen ist. Die Details zur Durchführung des Bereitschaftsdienstes werden in der Bereitschaftsdienstordnung (BO) der KVN und der Ärztekammer Niedersachsen festgelegt (Ärztekammer Niedersachsen, 2007).

Das Ziel der KVN ist es, „, ... die Patienten bis zur nächstmöglichen regulären 
ambulanten oder stationären Behandlung nach den Regeln der ärztlichen Kunst zweckmäßig, ausreichend und wirtschaftlich zu versorgen (....). Dabei arbeitet die KVN eng mit den übrigen für die Bereitschaftsdienstordnung verantwortlichen Organisationen zusammen, insbesondere Krankenhäusern, Transportorganisationen und Trägern des Rettungsdienstes"(Ärztekammer Niedersachsen, 2007, S. 1). Die Teilnehmer des Notdienstes werden in $\S 5$ der BO bestimmt. Es sind „alle zugelassenen Vertragsärzte sowie in medizinischen Versorgungszentren oder bei Vertragsärzten angestellte Ärzte, die in das Arztregister eingetragen sind", verpflichtet, am Notdienst mitzuwirken (Ärztekammer Niedersachsen, 2007, S. 4). Nach $\S 3$ BO können sie einem allgemeinen und/oder einem fachärztlichen Bereitschaftsdienst zugeteilt sein (Ärztekammer Niedersachsen, 2007). Der allgemeine Notdienst wird von niedergelassenen Ärzten aller Fachrichtigen besetzt. Am fachärztlichen Notdienst nehmen dagegen nur niedergelassene Ärzte eines bestimmten Fachgebietes teil. Welche Fachgebiete einen eigenen fachärztlichen Notdienst einzurichten haben, wird ebenfalls nach $\S 3$ BO festgelegt (Ärztekammer Niedersachsen, 2007).

Der ärztliche Notdienst kann auch von Krankenhäusern durchgeführt werden. Über Versorgungsverträge ( $§ 115$ Abs. 2 Nr. 3 SGB V) ist es möglich, dass die Kassenärztlichen Vereinigungen den Notdienst durch Kooperationen mit Krankenhäusern sicherstellen (§ 75 Abs. 1 S. 3 SGB V) (Luthe, 2013).

\subsubsection{Notdienstregelung in Göttingen}

Nach Informationen der $\mathrm{KVN}^{1}$ werden HNO-Notfälle je nach dem Schweregrad ihrer Erkrankung durch den allgemeinen Notdienst niedergelassener Ärzte oder durch die Universitätsmedizin Göttingen betreut. Die UMG ist für die Behandlung schwerwiegender HNO-Notfälle vorgesehen, während die leichten Fälle durch den allgemeinen Notdienst niedergelassener Ärzte behandelt werden.

Schwerwiegende Notfälle müssen häufig stationär betreut werden, weil nie-

\footnotetext{
${ }^{1}$ Nach schriftlicher Auskunft der Kassenärztlichen Vereingung Niedersachsen, Geschäftsstelle Göttingen vom 19.09.2011.
} 
dergelassene Ärzte nicht die notwendige Ausstattung vorhalten können. Nach Angaben der KVN haben andere Krankenhäuser - wie z. B. das Evangelische Krankenhaus in Weende - keine HNO-Klinik als Krankenhausabteilung. Die Belegärzte seien dort nur für die Betreuung der Belegpatienten verantwortlich. Ein HNO-Arzt sei damit nicht immer im Krankenhaus anwesend.

Daher wurde durch die KVN entschieden, dass ausschließlich der HNO-Notdienst der UMG die Behandlung dieser Fälle übernimmt. Es sei die einzige Klinik in Göttingen, die über eine HNO-Abteilung verfüge. Gravierendere Notfälle können hier zudem intensiver betreut werden, da auch stationäre Behandlungen möglich sind.

Die Anzahl der „leichten“ HNO-Notfälle sei zu gering, um einen eigenen HNONotdienst niedergelassener Ärzte zu legitimieren. Sie könnten auch durch den allgemeinen Notdienst versorgt werden, der von allen niedergelassenen Ärzten besetzt sei. Würde ein HNO-ärztlicher Bereitschaftsdienst angeboten werden, müssten die daran teilnehmenden HNO-Ärzte aus Göttingen sehr häufig einen Notdienst übernehmen. Die zeitliche Belastung könnte nur durch Bildung größerer Bezirke reduziert werden. Patienten hätten im Notfall deutlich weitere Anfahrtswege. Aus diesen Gründen habe man sich gegen eine solche Regelung ausgesprochen.

Die KVN gab weiterhin an, dass man einen fachärztlichen Notdienst zum Teil in anderen Spezialisierungsrichtungen finde, wie zum Beispiel in der Augenheilkunde, der flächendeckend in Niedersachsen vorhanden sei. Daneben gebe es in vielen Bezirken einen kinderärztlichen Notdienst. Bereitschaftsdienste in den anderen Fachbereichen seien dagegen nicht üblich. Göttingen stellt somit keine Ausnahme in der Notdienstregelung dar.

\subsubsection{Regionale Abweichungen der Notdienstregelungen}

Nicht überall in Deutschland wird ein fachärztlicher HNO-Notdienst ausschließlich durch die lokalen Kliniken sichergestellt. So wird beispielsweise in SchleswigHolstein ein zeitlich begrenzter fachärztlicher Notdienst durch niedergelassene HNO-Ärzte angeboten. Außerhalb der Notdienst-Zeiten besteht keine Bereit- 
schaft. In einfachen Fällen wird auf den allgemeinen Notdienst und bei schwerwiegenden Fällen auf die nächstgelegenen Kliniken mit HNO-Abteilung verwiesen. Analog zum allgemeinärztlichen Notdienst wird jede HNO-Praxis einem Notdienstbezirk zugeordnet, so dass immer eine Praxis aus je einem Bezirk Anlaufstelle für Notdienstpatienten ist (Kassenärztliche Vereinigung SchleswigHolstein, 2009). Im Vergleich zu den Regelungen in Göttingen zeigt dieses, dass es keine bundeseinheitliche Organisation im HNO-Notdienst gibt.

\subsubsection{HNO-Notdienst in der Universitätsmedizin Göttingen}

Anders als die regulären Patienten, die sich an der Leitstelle der HNO-Klinik anmelden, haben Notfallpatienten ihre erste Anlaufstelle in der allgemeinen Notaufnahme. Von dort werden sie auf die Stationen der HNO-Klinik weitergeleitet. Hier wird eine Reihenfolge der Behandlungen nach Dringlichkeit vom diensthabenden Assistenzarzt festgelegt. Die Patienten werden im Arztzimmer der Station untersucht. Ein standardisiertes Vorgehen der Assistenzärzte wird durch die Struktur des doppelseitigen Aufnahmebogens vorgegeben. Wenn es sich um einen schwerwiegenden Fall handelt bzw. eine OP-Indikation besteht, wird der Bereitschaftsdienst den Rufbereitschaftsdienst informieren, der von einem diensthabenden Oberarzt besetzt ist.

Mit einem Krankentransport ankommende Patienten werden in der allgemeinen Notaufnahme primär gesehen und registriert. Die „leichteren“ Fälle werden nach der Anmeldung in der allgemeinen Notaufnahme gebeten, die Station 6012 der HNO-Klinik eigenständig aufzusuchen.

\subsection{Motivation und Zielsetzung der Arbeit}

Nach den Regelungen der KVN sollen vor allem schwerwiegende HNO-Notfälle im HNO-Notdienst der UMG behandelt werden. Wie bereits in der Einleitung dargestellt wurde, werden derartige Notdienste oft von leichter erkrankten Patienten in Anspruch genommen.

Auf nationaler Ebene existieren lediglich zwei Untersuchungen (Universität 
Köln, Universität Jena), deren Ergebnisse nicht auf den HNO-Notdienst der UMG übertragen werden können (Bolz, 2009; Schlicht und Guntinas-Lichius, 2012). Für die Universität Göttingen hat es eine Analyse des HNO-Notdienstes bisher noch nicht gegeben. Um herauszufinden, wie sich die Situation im HNONotdienst der UMG gestaltet, soll in dieser Arbeit exemplarisch eine ausführliche Analyse des Notfallaufkommens an der HNO-Klinik der UMG im Jahr 2011 durchgeführt werden, um für die Ausrichtung und Steuerung des Notfallbereiches umfassende Beurteilungsgrundlagen zu generieren. Folgende Fragestellungen sollen hierbei zielführend sein:

1) Mit welchen Patientenzahlen hat es der universitäre HNO-Notdienst zu tun?

2) Wie viele Patienten werden ambulant und wie viele Patienten werden stationär therapiert?

3) Welche Diagnosen werden am häufigsten gestellt?

4) Welche Altersgruppen frequentieren besonders häufig den universitären HNO-Notdienst?

5) Aus welchem Umkreis suchen die Patienten den universitären HNO-Notdienst auf?

6) $\mathrm{Zu}$ welchen Zeiten suchen besonders viele Patienten den universitären HNO-Notdienst auf?

7) Welche Risikofaktoren und Komplikationen finden sich bei Notfall-Patienten nach Analyse der Anamnese, Diagnostik und Therapie der häufigsten ambulanten und stationären Diagnosen?

8) Wie stark interagiert der HNO-Notdienst mit anderen Abteilungen der Universitätsmedizin Göttingen? 


\section{4 Übersicht der Arbeit}

Im Anschluss an dieses einführende Kapitel werden in Kapitel zwei das Zahlenmaterial und statistische Methoden zur Auswertung vorgestellt. Im Kapitel drei werden die wichtigsten Ergebnisse zusammengefasst und schließlich in Kapitel vier diskutiert. 


\section{Material und Methoden}

\subsection{Mittelfristige Entwicklung der Patientenzahlen}

Zunächst wurde bestimmt, wie viele Patientenfälle für das Jahr 2011 auf den Stationen der HNO-Klinik registriert wurden. Um die Zahlen für das Jahr 2011 besser einordnen zu können, wurden die registrierten Behandlungen für das Vorund das Folgejahr ebenfalls bestimmt. Wie die Definitionen in den folgenden Abschnitten zeigen, sind nicht alle dieser registrierten Patienten nach unserer Definition „Notfälle“. Für den Fall, dass es sich nach unserer Definition nicht um einen Notfall handelte, wurden diese im weiteren Verlauf aus der Analyse ausgeschlossen.

\subsection{Einschlusskriterien}

Von den registrierten Patientenfällen wurden für das Jahr 2011 diejenigen Patienten retrospektiv für die weitere Analyse eingebunden, die zwischen 16:00 Uhr und 7:30 Uhr von montags bis donnerstags und von freitags von 16:00 Uhr bis montags 7:30 Uhr den Notdienst der HNO-Klinik aufgesucht haben und in den EDV-Systemen erfasst wurden. Der gewählte Zeitraum richtete sich nach den Öffnungszeiten der HNO-Poliklinik, die zu diesen Zeiten keine Patientenbehandlung anbietet.

\subsection{Datenquellen und Datentabelle}

Die Daten wurden aus dem sogenannten SAP- und ixserv-System und aus Patientenakten erhoben. Das System $S A P$ wird in der UMG zur patientenbe- 
zogenen Dokumentation und Abrechnung verwendet. In der UMG ist die für Krankenhäuser entwickelte $S A P$-Version $S A P I S-H$ im Einsatz.

Die Daten aus den oben genannten Quellen wurden mit Hilfe des Computerprogramms Microsoft Excel@ in anonymisierter Form analysiert.

\subsection{Umfang der Analyse}

Von den 2.699 Fällen des Jahres 2011 wurden nur 2.396 Fälle betrachtet. Es mussten 303 Fälle aus der Analyse ausgeschlossen werden. Dafür gab es zwei Gründe: Zum einem wurden nicht alle aufgelisteten Patienten in der vorliegenden Arbeit als „Notfall“ gezählt, da sie außerhalb des definierten Zeitrahmens behandelt wurden und somit aus Sicht dieser Analyse falsch zugeordnet waren und zum anderen waren nur unvollständige Angaben zu einem Teil der Patienten vorhanden, so dass keine Identifizierung in den Systemen SAP und ixserv möglich war (vgl. Abschnitt 1.1).

\subsection{Aufbau der Analyse}

Nach der Bestimmung der mittelfristigen Patientenzahlen und des Umfanges der Analyse, erfolgt im Teil „Generelle Patientendaten“ (vgl. Abschnitt 3.2) die Auswertung der Aufnahmeart, der Diagnosen, der Altersstruktur, des Einzugsgebiet und der Stoßzeiten. In dem folgenden Teil „Spezielle Patientendaten“ (vgl. Abschnitt 3.3) wurden häufig vorkommende Notfälle der ambulanten und stationären Patienten nach medizinischen Parametern detaillierter untersucht. Dafür wurden Daten der Anamnese, des Befundes und der Therapie erhoben und ausgewertet. Es wurde insbesondere ermittelt, ob Risikofaktoren und Komplikationen vermerkt waren. Zudem wurden die Häufigkeiten der invasiven Eingriffe, Weiterbehandlungen und konsiliarischen Untersuchungen analysiert. 


\subsection{Generelle Patientendaten}

\subsubsection{Aufnahmeart}

Die Patienten wurden in dieser Analyse danach eingeteilt, ob sie ambulant oder stationär behandelt wurden. Als stationär galt ein Patient, wenn dieser entweder nach der Untersuchung im HNO-Notdienst stationär aufgenommen wurde oder bereits zum Zeitpunkt der Untersuchung stationär in einer Klinik der Universitätsmedizin Göttingen aufgenommen war. Insgesamt wurden 2094 ambulante und 302 stationäre Fälle in der vorliegenden Arbeit analysiert.

\subsubsection{Diagnosen}

Für jeden Patienten waren die Diagnosen im SAP-System in kodierter Form hinterlegt. Die Kodierung erfolgte dort nach der „Internationalen statistischen Klassifikation der Krankheiten und verwandter Gesundheitsprobleme" aus dem Jahr 2008 (ICD-10) (Hoppe et al., 2008). Die Diagnosen wurden für diese Arbeit mit Hilfe des ICD-10-Klassifikationssystems weiter zusammengefasst. Des weiteren wird aufgelistet, welche Diagnosenbezeichnung für diese Arbeit verwendet wurde (vgl. Tabelle 6 im Anhang).

Bei den ambulanten Patienten wurden alle der ein bis drei Diagnosen pro Patientenfall aus dem SAP-System erhoben. Handelte es sich um einen stationären Fall, wurde aufgrund der zu großen Datenmenge eine Auswahl von Diagnosen getroffen. In der Untersuchung wurden nur die Diagnosen ausgewertet, die dem Gebiet der Hals-, Nasen-, und Ohrenheilkunde zuzuordnen waren.

\subsubsection{Altersstruktur}

Das Alter der Patienten wurde aus dem SAP-System bestimmt. Um mehr Übersichtlichkeit für die graphische Darstellung der Altersstruktur zu erreichen, wurden die Patienten in Gruppen (Gr.) zusammen gefasst, die jeweils eine halbe Dekade umfassten. Um Trends formulieren zu können, wurden breitere Klassen gebildet. 


\subsubsection{Einzugsgebiet}

Weiterhin wurde untersucht, aus welchen Gebieten die Patienten den HNONotdienst aufsuchten. Um das Einzugsgebiet der Patienten zu bestimmen, wurden die Erstwohnsitze aller Patienten aus dem SAP-System ausgewertet. Es wurden für eine bessere Übersichtlichkeit Gruppen nach den ersten drei Ziffern der Postleitzahlen gebildet. Die letzten beiden Ziffern wurden nicht weiter aufgeschlüsselt und durch ein „X“ dargestellt. Die Ergebnisse dieser Untersuchung wurden verglichen mit den Einwohnerzahlen, die das Statistische Bundesamt für das Jahr 2011 ermittelt hat. Das statistische Bundesamt listet u. a. für jedes Postleitzahlengebiet in Deutschland die Ergebnisse der Einwohnerzählungen auf $^{2}$. Damit die Zahlen des statistischen Bundesamtes mit den Zahlen unserer Analyse für das Einzugsgebiet der Patienten verglichen werden konnten, wurden die Zahlen des Statistischen Bundesamtes ebenfalls für die gleichen Gruppen zusammengefasst (370XX, 371XX, 372XX, 373XX, 374XX, 375XX, 376XX), d. h. es wurden alle Zählungen der einzelnen Postleitzahlengebiete, die zu einer der oben genannten Gruppen gehörten, z. B. 370XX, aufsummiert.

\subsubsection{Stoßzeiten}

Die Stoßzeiten im HNO-Notdienst wurden für den Jahres-, Wochen- und Tagesverlauf ermittelt. Grundlage für die Auswertung war der Aufnahmezeitpunkt. Der Aufnahmezeitpunkt wurde als der Zeitpunkt definiert, an dem sich der Notdienst-Patient auf der Ebene 01 (Allgemeine Notaufnahme der UMG) erstmalig angemeldet hat. Für die stationären Patienten, die bereits zum Zeitpunkt des Notdienst-Besuches angemeldet waren, wurde der Anmeldungszeitpunkt in der HNO-Klinik ausgewertet.

\footnotetext{
${ }^{2}$ Nach Informationen des Statistischen Bundesamtes vom 31.12.2011: https:

//www.destatis.de/DE/ZahlenFakten/LaenderRegionen/Regionales/

Gemeindeverzeichnis/Administrativ/AdministrativeUebersicht.html
} 


\subsection{Spezielle Patientendaten}

\subsubsection{Risikofaktoren}

Um Risikofaktoren bestimmen zu können, wurden exemplarisch für die fünf häufigsten Diagnosen der stationären und für die zehn häufigsten Diagnosen der ambulanten Patienten Auswertungen zur Anamnese, zum Befund, zur Diagnostik, zur Therapie und zum weiteren Verlauf vorgenommen. Die Angaben hierzu wurden aus dem Aufnahmebogen, den Arztbriefen und dem ixserv-System und SAP-System erhoben. Bei den stationären Patienten wurden alle Patientenfälle der fünf häufigsten Diagnosen untersucht. Bei den ambulanten Patienten wurden aufgrund der höheren Fallzahl jeweils 50 Fälle pro Diagnose untersucht. Die 50 Fälle wurden zufällig ausgewählt.

Um mögliche Zusammenhänge zwischen bestimmten Altersgruppen und Diagnosen herstellen zu können, wurden Median, Mittelwert und Standardabweichung für die Altersangaben der Patienten bestimmt. Die Daten hierfür stammen aus $S A P$.

Aus Gründen der Übersichtlichkeit wurden nicht für alle der oben genannten häufigsten Diagnosen Risikofaktoren im Kapitiel „Ergebnisse“ dargestellt, sondern exemplarisch für jeweils die häufigste Diagnose der ambulanten und stationären Patienten und für die Diagnose, die am häufigsten zu einem ungeplanten, invasiven Eingriff geführt hat.

Daraufhin wurde bei den Epistaxis-Patienten die Anamnese näher untersucht. Festgelegte Allgemeinerkrankungen wurden als Risikofaktoren bestimmt. Das Auftreten dieser Risikogruppen (HK-, Lungen-, Nieren- und Lebererkrankungen, Diabetes, Drogenkonsum und eine vorangegangene Nasen-OP) wurde analysiert. Um mehr Übersichtlichkeit zu erhalten, wurden einzelne Erkrankungen in Gruppen zusammengefasst. Die Einnahme von Antikoagulanzien wurde ebenfalls durch die Angaben aus der Anamnese untersucht. 


\subsubsection{Anzahl der Operationen der häufigsten Erkrankungen}

Die Anzahl der Operationen wurde ausschließlich für die stationären Patienten bestimmt. Die Daten stammen aus dem SAP-System.

\subsubsection{Besonderheiten der Diagnose „Nachblutungen“}

Für die Diagnose „Nachblutungen“ wurde bestimmt, ob der Eingriff, der zu der Nachblutung geführt hat, in einem externen Krankenhaus oder im Universitätsklinikum Göttingen vorgenommen wurde. Die Angaben hierzu stammen abermals aus der Anamnese der Patienten. Wie lange der Eingriff zurücklag, wurde ebenfalls aus der Anamnese erhoben. Welche Form der Nachblutung vorlag, konnte aus dem SAP-System erhoben werden, in dem die Diagnosen weiter differenziert vorlagen (s. o.). Die Art der Behandlung konnte aus dem Aufnahmebogen und den Patientenakten entnommen werden. Es wurde zwischen invasiver und konservativer Therapie unterschieden.

\subsubsection{Konsile}

Die Aufnahmeart und die Konsiliaruntersuchungen wurden alleine für die stationären Patienten bestimmt. Die „Aufnahmeart“ wurde in drei verschiedene Kategorien eingeteilt, in die jeder stationäre Fall eingeordnet werden konnte:

Gruppe 0: Stationäre Aufnahme durch die HNO-Klinik

Gruppe 1: Stationäre Aufnahme durch eine andere Klinik der UMG mit anschließender Überweisung in die HNO

Gruppe 2: Erste Untersuchung in der HNO-Klinik mit anschließender Überweisung in eine andere Klinik der UMG

In die Analyse der Überweisungen (vgl. Tabelle 3.5) wurden alle Patienten einbezogen, die von einer anderen Klinik der UMG in die HNO überwiesen wurden (Gruppe 1) oder umgekehrt (Gruppe 2). Die Verteilung dieser Patientenzahl auf die einzelnen Kliniken wurde im Anschluss in absoluten (abs.) und relativen (rel.) Häufigkeiten dargestellt. Die Summe der absoluten und relativen Häufigkeiten für die jeweiligen Kliniken wurde ebenfalls errechnet (vgl. 
„Gesamt Gr. 1 und Gr. 2 (abs.)“ und „Gesamt Gr. 1 und und Gr. 2 (in \%)“ in Tabelle 3.5), um das Ausmaß der Interaktion der einzelnen Kliniken bewerten zu können. Die Daten dafür stammen aus dem SAP-System. Jede Bewegung der Patienten wurde dort mit Datum, Uhrzeit und Angabe der Klinik dokumentiert.

\subsubsection{Nachsorge}

Für die ersten zwei Monate nach der Behandlung im Notdienst wurde untersucht, wie viele der Patienten erneut ambulant und stationär behandelt wurden und erneut als „Notfall" registriert waren. Daneben wurde im SAP-System bestimmt, ob im Falle eines Wiederkehrens ein invasiver Eingriff vorgenommen wurde. Die Daten dazu stammen aus dem SAP-System.

\subsection{Statistische Auswertung}

Die statistische Auswertung der Daten wurde mit dem Programm Statistica(C) (Version 10) in Zusammenarbeit mit dem Institut für Medizinische Statistik der Universitätsmedizin Göttingen angefertigt. Es wurden abhängig vom Endpunkt absolute und relative Häufigkeiten bzw. Mittelwert und Standardabweichung bestimmt. Das Einzugsgebiet wurde mit dem $\chi^{2}$-Test untersucht. Es wurde überprüft, ob die Verteilungsunterschiede als signifikant anzusehen waren oder nicht. Für diese statistischen Testverfahren wurde ein Signifikanzniveau von $\alpha$ $=0,05$ angewendet. Das bedeutet, dass ein p-Wert $<0,05$ auf eine statistische Signifikanz hindeutete.

Die graphische Darstellung der Ergebnisse erfolgte in Form von Kreis-, Säulenund Liniendiagrammen, die mit Microsoft Excel(c) (2011) angefertigt wurden. Daneben wurden Boxplots mit Statistica(C) (Version 10) angefertigt. In dieser Abbildungsform werden in einer Fünf-Punkt-Zusammenfassung Median, zwei Quartile und beide Extremwerte zusammengefasst. Im Box-Plot werden in einer kleinen Box der Median markiert und in der darum befindlichen größeren Box der Bereich dargestellt, in dem sich die mittleren $50 \%$ der Daten aufhalten. Dabei wird die Länge der Box als Interquartilsabstand (IQR) definiert. Oben 
und unten an der Box erfolgt die Begrenzung durch die Quartile. Zwei Linien verlängern die Box, die als Whisker bezeichnet werden. Durch diese Whisker werden die außerhalb der Box liegenden Werte repräsentiert. Diese Whisker sollen dabei nicht länger als 1,5 x IQR sein. Die Whisker enden bei dem Wert, der noch innerhalb der Grenze liegt. Bis 3 x IQR wird von milden und über 3 x IQR wird von extremen Ausreißern gesprochen. 


\section{Ergebnisse}

\subsection{Mittelfristige Entwicklung der Patientenzahlen und Umfang der Analyse}

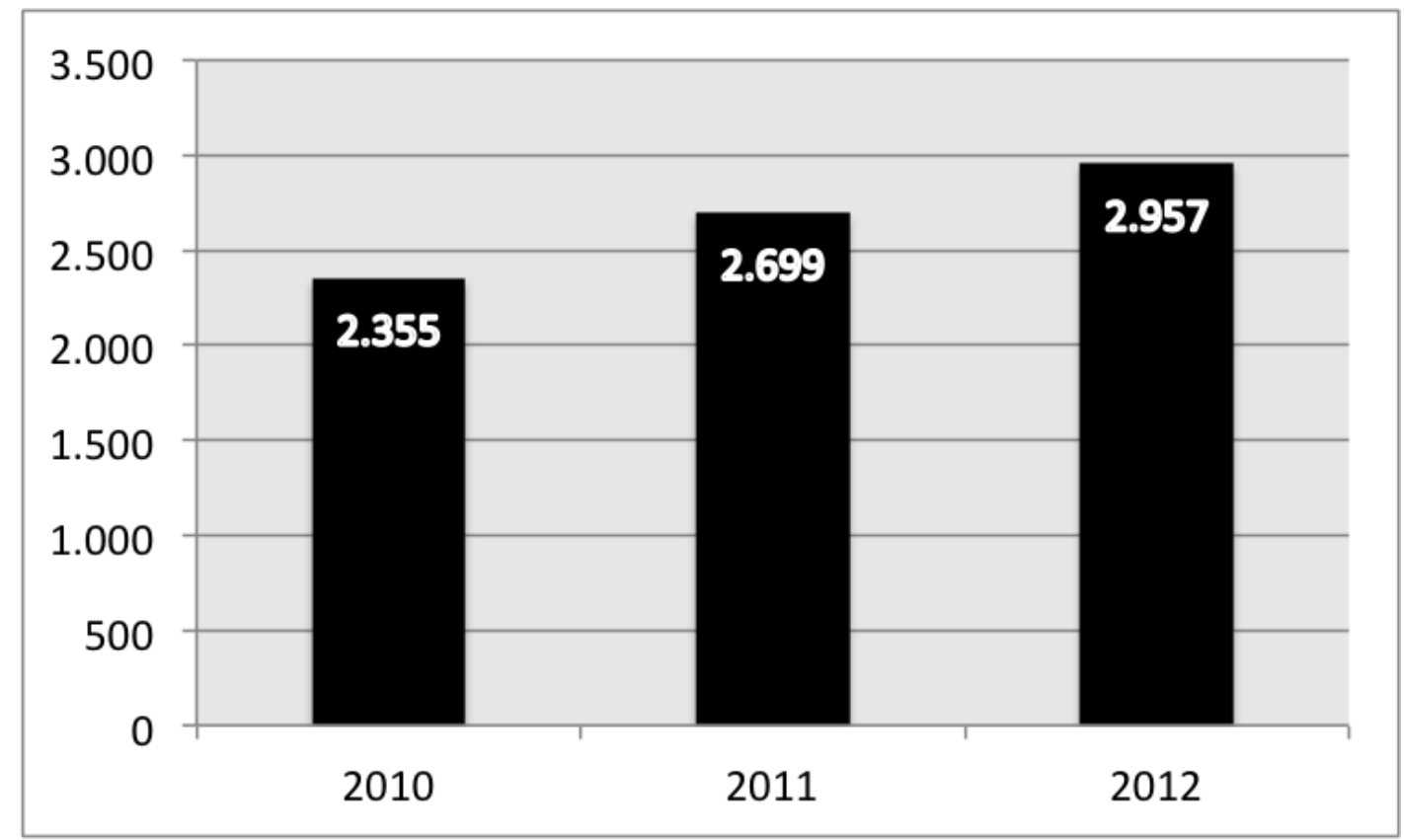

Abbildung 3.1: Registrierte Patientenzahlen (n) der Jahre 2010, 2011 und 2012 auf Station 6012

In den letzten drei Jahren wurde ein kontinuierlicher Anstieg der Patientenzahlen verzeichnet ${ }^{3}$. Im Jahr 2010 wurden 2.355 Fälle registriert. Ein Anstieg von 14,6 \% wurde im Jahr 2011 beobachtet (2.699 Patienten). Noch mehr Patienten wurden 2012 gegenüber dem Jahr 2010 behandelt. Gegenüber dem Jahr

${ }^{3}$ Dabei handelt es sich um alle registrierten Patienten der Station 6012. Diese sind nach unserer Definition nur zu einem Teil „Notfälle“ (vgl. 2.1 ff.) 
2010 lag eine Steigerung von 25,6 \% vor (vgl. Abb. 3.1). Von den 2.699 Fällen des Jahres 2011 wurden nur 2.396 Fälle in der weiteren Analyse betrachtet und damit als „Notfälle“ gewertet.

\subsection{Generelle Patientendaten}

\subsubsection{Aufnahmeart}

Ob Patienten intensiv im Notdienst therapiert werden mussten oder nicht, wurde daran festgemacht, ob eine stationäre oder ambulante Behandlung notwendig war. Es zeigte sich, dass im Notdienst 87,4 \% bzw. 2094 Patienten ambulant therapiert wurden. Ein geringerer Teil von 12,6 \% (302 Patienten) wurde stationär aufgenommen bzw. war bereits in der HNO-Klinik oder einer anderen Klinik der UMG in stationärer Therapie. Dies entspricht einem Verhältnis von ambulanten zu stationären Patienten von sieben zu eins (vgl. Abb. 3.2).

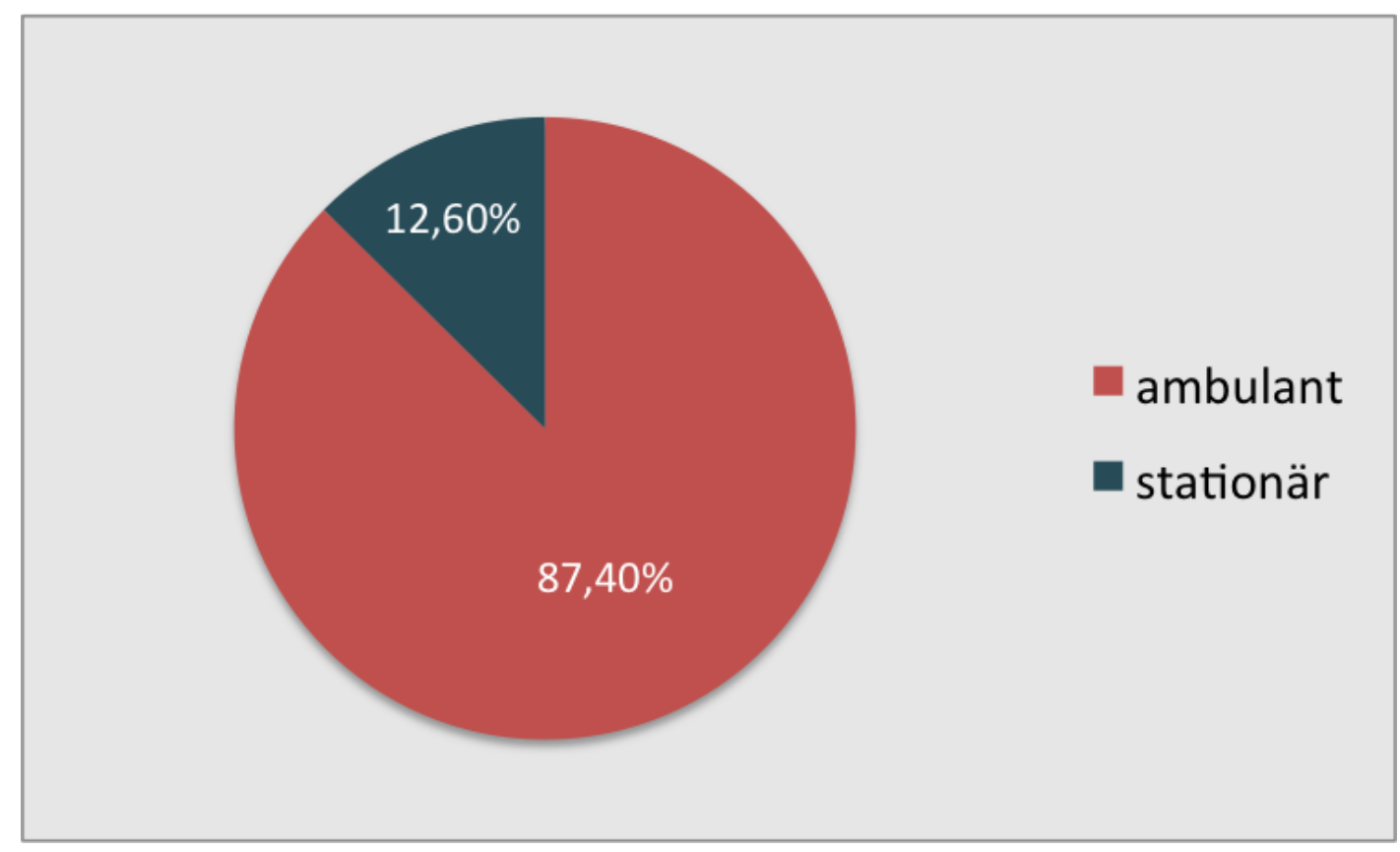

Abbildung 3.2: Verhältnis der ambulanten zu den stationären Patienten (in \%) 


\subsubsection{Diagnosen}

Es sollte eine Übersicht darüber entstehen, mit welchen Krankheitsfällen der Notdienst besonders häufig konfrontiert wird. Dafür wurden die Diagnosen untersucht, die für jeden Patientenfall elektronisch hinterlegt waren. Pro Patient wurden bis zu drei Diagnosen erhoben, so dass die Anzahl der Diagnosen über der Anzahl der Patienten lag. Insgesamt wurden dadurch 2.350 Diagnosen für alle 2.094 ambulanten Patienten erhoben und 365 Diagnosen für 302 stationäre Patienten.

Die zehn häufigsten Diagnosen der ambulanten Patienten machen $54 \%$ aller ambulanten Diagnosen aus (vgl. Tabelle 3.1); bei den stationären Patienten werden durch die häufigsten fünf Diagnosen 32,9 \% aller stationären Diagnosen repräsentiert (vgl. Tabelle 3.2). Sowohl bei den ambulanten als auch bei den stationären Diagnosen ist „Epistaxis“ (Nasenbluten) die häufigste Erkrankung. Auf diese Erkrankung entfielen 11,4\% aller ambulanten und 10,1\% aller stationären Diagnosen (vgl. Tabelle $3.1 \mathrm{f}$.).

Die Plätze zwei bis fünf der häufigsten ambulanten Diagnosen waren ausschließlich Erkrankungen des Ohres (vgl. Tabelle 3.1). Auf den Plätzen sechs bis zehn befinden sich die „akute Pharyngitis“, „Tinnitus aurium“, „Fremdkörper in den Atemwegen“, „akute Tonsillitis“ und die „chronische Sinusitis“ (vgl. Tabelle 3.1).

Bis auf die häufigste Diagnose „Epistaxis“ unterscheiden sich die Plätze zwei bis fünf bei den stationären Patienten von denen der ambulanten Patienten (vgl. Tabelle 3.1 f.). Ein häufiger Behandlungsgrund sind zudem die Erkrankungen der Tonsillen. Denn die zweithäufigste Diagnose der stationären Patienten war der „Peritonsillarabszess“, die dritthäufigste Diagnose waren „Nachblutungen", von denen 95,5\% nach einer zuvor erfolgten Tonsillektomie auftraten. Die fünfthäufigste Diagnose lautete mit einem Anteil von 3,3\% „chronische Erkrankung der Tonsillen“ (vgl. Tabelle 3.2). 
Tabelle 3.1: Die zehn häufigsten Diagnosen (in absoluten und relativen Zahlen) der ambulanten Patienten

\begin{tabular}{|c||c|c|}
\hline Diagnosen & Abs. (n) & Rel. (\%) \\
\hline \hline „Epistaxis“ & 268 & 11,4 \\
\hline „Eitrige Otitis media“ & 205 & 8,7 \\
\hline "Otitis externa“ & 195 & 8,3 \\
\hline „Zeruminalpfropf“" & 134 & 5,7 \\
\hline "Hörverlust/Hörsturz“ & 114 & 4,9 \\
\hline "Akute Pharyngitis“ & 90 & 3,8 \\
\hline "Tinnitus aurium“" & 84 & 3,6 \\
\hline „Fremdkörper Atemwege“ & 67 & 2,9 \\
\hline "Akute Tonsillitis“ & 60 & 2,6 \\
\hline "chronische Sinusitis“ & 51 & 2,2 \\
\hline
\end{tabular}

Tabelle 3.2: Die fünf häufigsten Diagnosen (in absoluten und relativen Zahlen) der stationären Patienten

\begin{tabular}{|c||c|c|}
\hline Diagnosen & Abs.(n) & Rel. (\%) \\
\hline \hline „Epistaxis“ & 37 & 10,1 \\
\hline "Peritonsillarabszess" & 27 & 7,4 \\
\hline "Nachblutungen“ & 22 & 6,0 \\
\hline „Störungen der Vestibularfunktion" & 22 & 6,0 \\
\hline "chronische Krankheiten der Tonsillen“ & 12 & 3,3 \\
\hline
\end{tabular}




\subsubsection{Altersstruktur}

\section{Altersverteilung der ambulanten und stationären Patienten im Vergleich zur Altersstruktur der Stadt Göttingen}

Es stellte sich die Frage, welche Altersgruppen den Notdienst aufsuchten und wie sich das Alter der Notdienstpatienten von der Göttinger Bevölkerung unterschied.

Die Altersgruppe der 21 bis 25 Jahre alten Patienten hatte bei den ambulanten Patienten die höchsten Patientenzahlen. Die jungen Erwachsenen und Personen mittleren Alters waren bei den ambulanten Patienten insgesamt stark vertreten (vgl. Abb. 3.3). Es waren 34,1 \% (714 Patienten) zwischen 21 und 40 Jahren alt (davon entfallen alleine 21,3 \% bzw. 445 Patienten auf die Gruppe zwischen 21 und 30 Jahren). Diese Jahrgänge waren auch in der allgemeinen Altersstruktur Göttingens besonders stark vertreten (vgl. Abb. 6.1 f. im Anhang). Die zweitstärkste Altersgruppe umfasst die Patienten, die zwischen 41 und 60 Jahren alt sind (vgl. Abb. 3.3). Hier waren es 26,6\%.

Bei den stationären Patienten war die Altersgruppe der 21- bis 40-jährigen ebenfalls mit 76 Patienten (25,2 \%) stark vertreten, jedoch ist der Anteil deutlich niedriger als bei den ambulanten Patienten (vgl. Abb. 3.3). Die Gruppen der Kleinkinder von null bis fünf Jahren mit 28 Fällen (9,3\%) und die der 71- bis 75-jährigen Patienten (Obergruppe: 61- bis 80-jährige Patienten) mit 31 Fällen (10,3\%) von insgesamt 302 stationären Notfällen sind dagegen im Vergleich zu den ambulanten Patienten und zur Altersstruktur Göttingens überproportional häufig vertreten (vgl. Abb. 3.3 und 6.1 f. im Anhang). Folglich liegt eine abweichende Altersverteilung im Vergleich zur Alterspyramide Göttingens vor. 


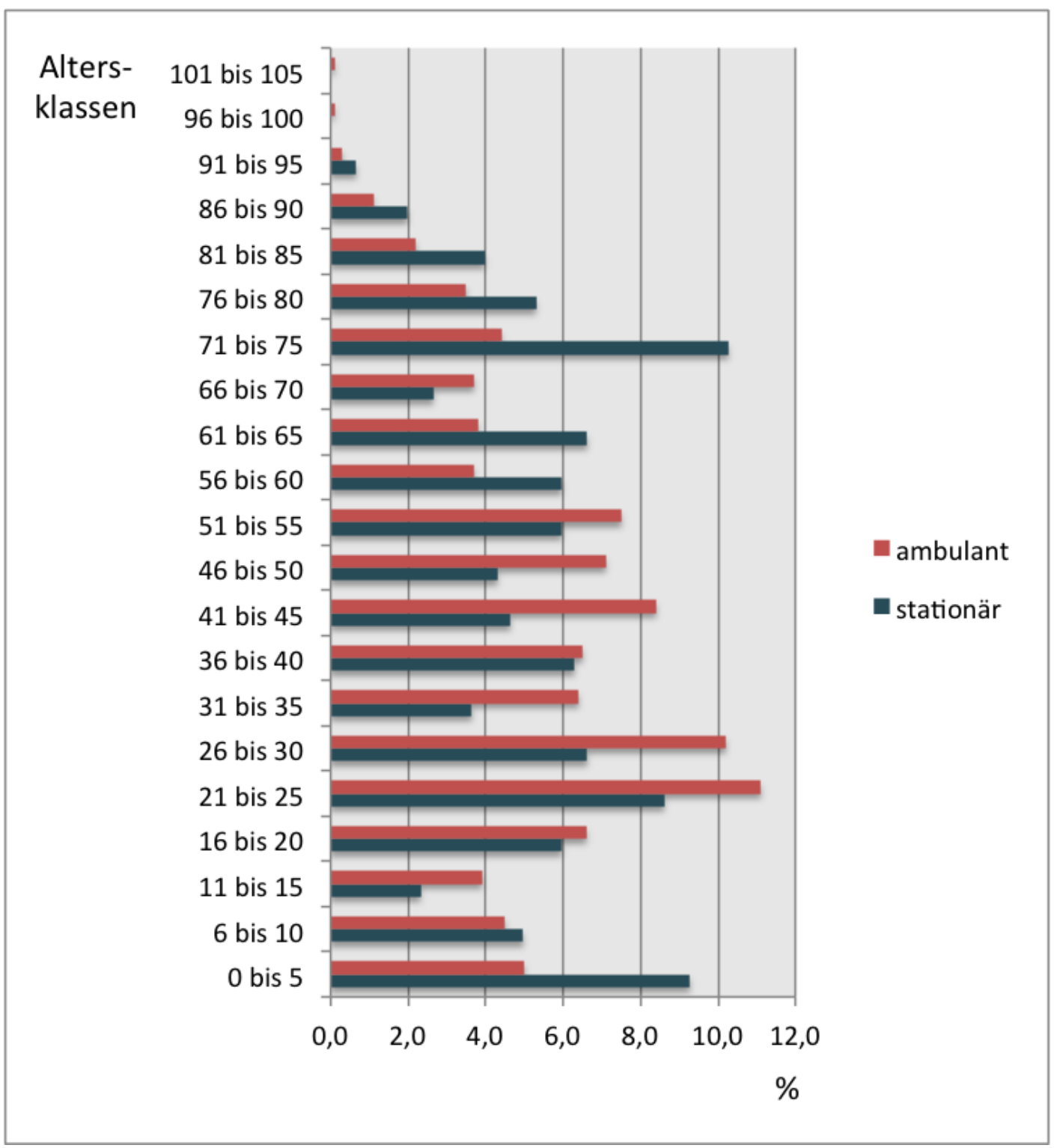

Abbildung 3.3: Häufigkeiten (in \%) der ambulanten (rot) und stationären (grün) Patienten in Altersklassen (in Jahren); Mittelwert für die ambulanten Patienten: 39,4 Jahre und Standardabweichung 21,99 Jahre; Mittelwert für die stationären Patienten: 43,4 Jahre und Standardabweichung 25,77 Jahre 


\subsubsection{Einzugsgebiet}

Tabelle 3.3: Einwohnerzahlen nach Postleitzahlengruppen nach Informationen des Statistischen Bundesamtes vom 31.12.2011 (Quelle: vgl. Abschnitt 3.2.4)

\begin{tabular}{|c||c|}
\hline Postleitzahlengebiet & Einwohnerzahl \\
\hline \hline $370 X X$ & 121.364 \\
\hline $371 X X$ & 174.134 \\
\hline $372 X X$ & 95.191 \\
\hline $373 X X$ & 104.549 \\
\hline $374 X X$ & 52.338 \\
\hline $375 X X$ & 91.888 \\
\hline $376 X X$ & 127.373 \\
\hline
\end{tabular}

Die regionale und überregionale Bedeutung des HNO-Notdienstes wurde anhand der Auswertung der Postleitzahlen bestimmt. In Tabelle 3.3 werden zunächst die Einwohnerzahlen dargestellt, die sich nach den Zählungen des Statistischen Bundesamt ergeben ${ }^{4}$. Diese Zahlen werden im Folgenden zum Vergleich mit unseren Ergebnissen herangezogen.

Aus der Stadt Göttingen (Postleitzahlen: 370XX) kamen mit 38,0 \% (797 Fälle) die meisten der ambulanten Notfall-Patienten, obwohl die Einwohnerzahl in diesem Postleitzahlengebiet nur am dritthöchsten war (vgl. Tabelle 3.3). Der an die Stadt Göttingen direkt angrenzende Bezirk (Postleitzahlen: 371XX) verzeichnete ebenfalls hohe Patientenzahlen (682 Fälle bzw. 32,6 \%) (vgl. Abb. 3.4). Patienten aus anderen in Abb. 3.4 verzeichneten Bezirken frequentierten die HNO-Klinik in deutlich geringerem Umfang. Insgesamt kamen 89,3\% aus

\footnotetext{
${ }^{4}$ Nach Informationen des Statistischen Bundesamtes vom 31.12.2011: https:

//www.destatis.de/DE/ZahlenFakten/LaenderRegionen/Regionales/

Gemeindeverzeichnis/Administrativ/AdministrativeUebersicht.html
} 
dem dargestellten Gebiet (vgl. Abb. 3.4). Die verbleibenden Patienten (10,7 \%) waren aus anderen Teilen Deutschlands und aus dem Ausland.

Im Vergleich dazu kamen 26,8 \% (81 Fälle) der stationären Patienten aus der Stadt Göttingen (Postleitzahlen: 370XX). Anders als bei den ambulanten Patienten suchten bei den stationären Patienten mit 31,8 \% (96 Fälle) jedoch die meisten Patienten aus dem an die Stadt Göttingen direkt angrenzenden Gebiet (Postleitzahlen 371XX) den Notdienst auf. Die Bevölkerungszahlen sind in diesem Teil am höchsten (vgl. Tabelle 3.3). Es kamen insgesamt 84,4 \% (255 Patienten) aus dem dargestellten Gebiet (vgl. Abb. 3.5). Von außerhalb dieser Region kamen 15,6 \% bzw. 47 Patienten.

Der $\chi^{2}$-Homogenitätstest zeigte, dass nur aus den Gebieten mit den Postleitzahlen 370XX ( $\chi^{2}: 15,229$ und p-Wert: 0,0001), 375XX ( $\chi^{2}: 16,168$ und p-Wert: 0,0001) und 376XX ( $\chi^{2}: 11,700$ und p-Wert: 0,0006) ambulante und stationäre Patienten signifikant unterschiedlich häufig den HNO-Notdienst aufsuchten. 


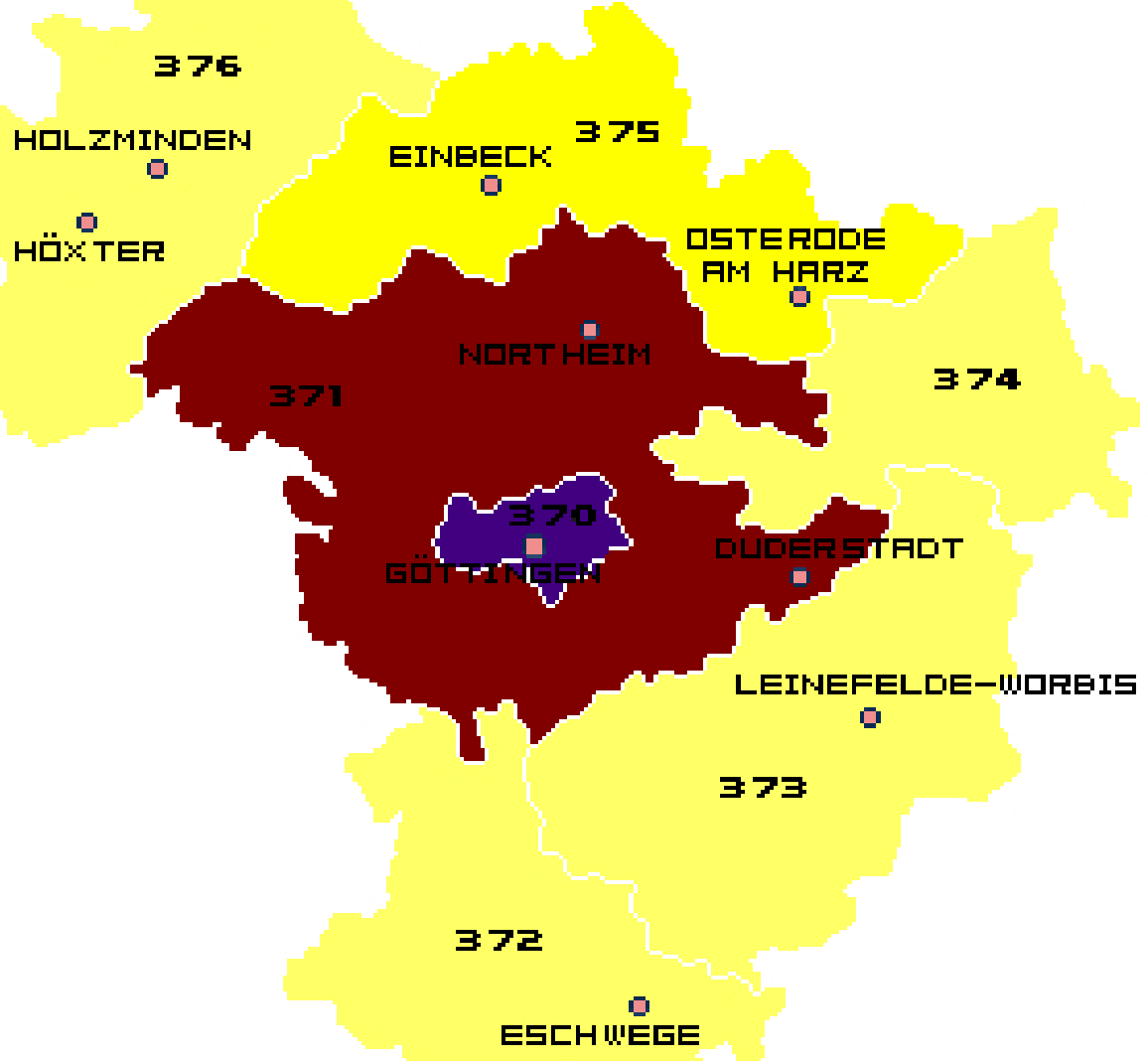

\begin{tabular}{|l|l|}
\hline Farbe & $\%$ \\
\hline & 0 bis 4,9 \\
\hline & 5 bis 9,9 \\
\hline & 10 bis 14,9 \\
\hline & 15 bis 19,9 \\
\hline & 20 bis 24,9 \\
\hline & 25 bis 29,9 \\
\hline & 30 bis 34,9 \\
\hline & 35 bis 39,9 \\
\hline
\end{tabular}

Abbildung 3.4: Einzugsgebiet der ambulanten Patienten (Patientenanteil in \%) 


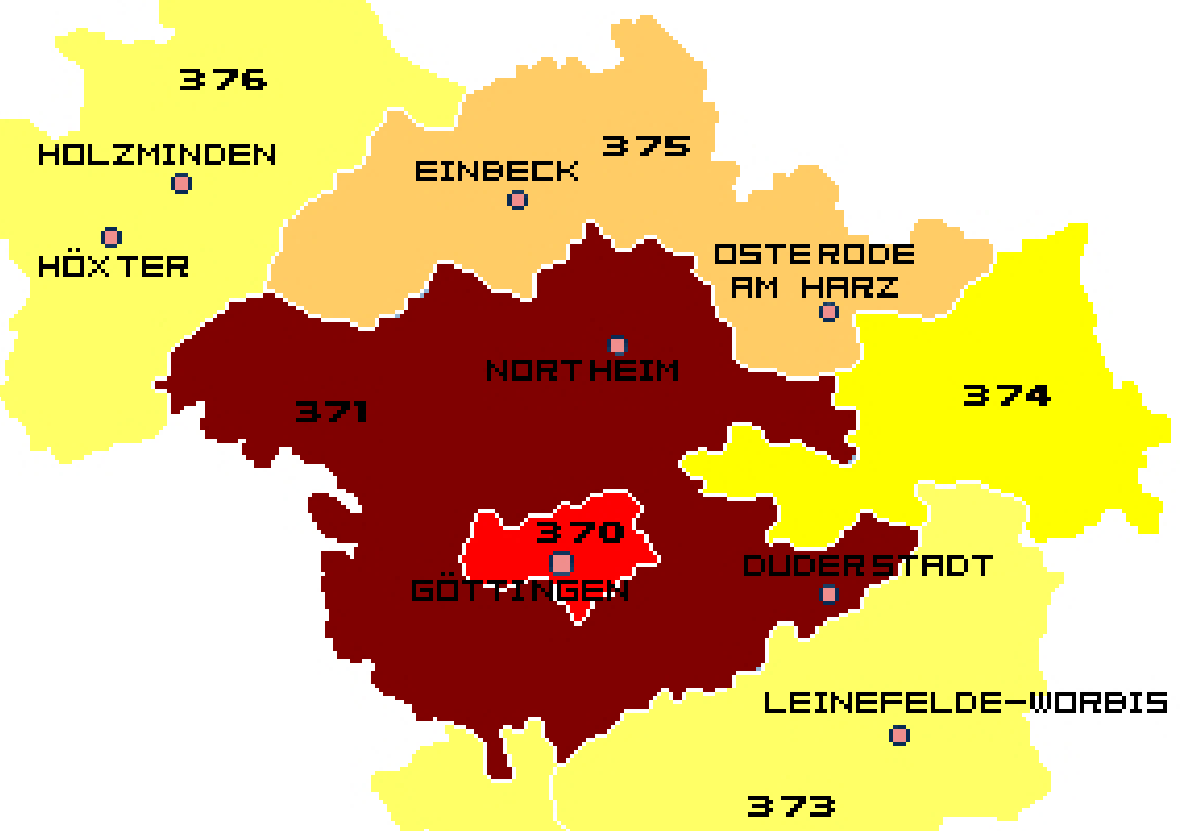

$\exists>$ 르

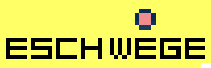

\begin{tabular}{|l|l|}
\hline Farbe & \% \\
\hline & 0 bis 4,9 \\
\hline & 5 bis 9,9 \\
\hline & 10 bis 14,9 \\
\hline & 15 bis 19,9 \\
\hline & 20 bis 24,9 \\
\hline & 25 bis 29,9 \\
\hline & 30 bis 34,9 \\
\hline & 35 bis 39,9 \\
\hline
\end{tabular}

Abbildung 3.5: Einzugsgebiet der stationären Patienten (Patientenanteil in \%) 


\subsubsection{Stoßzeiten}

Um herauszufinden, ob der Notdienst über ausgewählte Zeiträume gleichmäßig von Patienten aufgesucht wurde oder ob Schwankungen hinsichtlich der Patientenzahlen festzustellen waren, wurde der Aufnahmezeitpunkt der Patienten ausgewertet. Die Stoßzeiten werden im Folgenden im Jahres- und Wochenverlauf dargestellt.

\section{Jahresverlauf}

Die Patientenzahlen der ambulanten Patienten unterlagen saisonalen Schwankungen. Ein großer Teil der Patienten suchte im Frühjahr den Notdienst auf. Speziell im Monat Mai wurde die HNO-Klinik der UMG häufig frequentiert, als 209 bzw. 10 \% aller ambulanten Patienten die Klinik aufsuchten (vgl. Abb. 3.6). Die fünf häufigsten Diagnosen im Mai waren die „eitrige Otitis media“ (21 Fälle bzw. 10\% von allen Diagnosen des Monats Mai), „Epistaxis“ (19 Fälle bzw. 9,1 \%), „Hörverlust/Hörsturz“ (18 Fälle bzw. 8,6 \%), „Otitis externa“ (16 Fälle bzw. 7,7 \%) und die „Frakturen des Schädels“ (14 Fälle bzw. 6,7 \%). Die ersten vier Diagnosen („eitrige Otitis media“, „Epistaxis“, „Hörverlust/Hörsturz“ und „Otitis externa“) liegen auch auf das ganze Jahr gesehen in der Häufigkeit vorne (vgl. Tabelle 3.1). Die „Frakturen des Schädels“ kommen dagegen im Mai häufiger als in der Jahresstatistik der Diagnosen vor (vgl. Tabelle 3.1). Zu diesen „Frakturen des Schädels" zählen u. a. die „Nasenbeinfrakturen“ und die „Frakturen des Orbitabodens“. Im Monat Mai sind 13 von 14 Frakturfällen (92,9 \%) auf Nasenbeinfrakturen zurückzuführen und nur ein Fall ging auf eine Fraktur des Orbitabodens zurück. Im April und im Juni war die Patientenanzahl mit 205 bzw. 206 Fällen (je 9,8 \%) nur geringfügig niedriger. Nach den stark frequentierten Monaten im Frühahr nahmen die Notdienstbesuche wieder ab. Im September kamen die wenigsten Patienten in den Notdienst (120 Patienten bzw. 5,7 \%). Zu einem erneuten Anstieg der Patientenzahlen kam es wieder im Oktober und Dezember (199 und 192 Patienten im Monat, vgl. Abb. 3.6).

Die Notfälle bei den stationären Patienten unterlagen ebenfalls Schwankungen. Die höchsten Patientenzahlen wurden im Juli mit 35 Patienten (11,6 \%) vor- 
gefunden. Am zweithäufigsten kamen die Patienten im Februar (32 Patienten bzw. 10,6 \%), gefolgt vom Januar, April, Mai und Oktober. Hier nahmen je 29 Patienten bzw. 9,6 \% den Notdienst der Klinik in Anspruch. Die geringsten Patientenzahlen waren, genauso wie bei den ambulanten Patienten, im September zu verzeichnen (13 Patienten bzw. 4,3 Prozent). Aufgrund der geringen Fallzahlen ist es schwierig, von saisonalen Trends bei den stationären Patienten zu sprechen (vgl. Abb. 3.6).

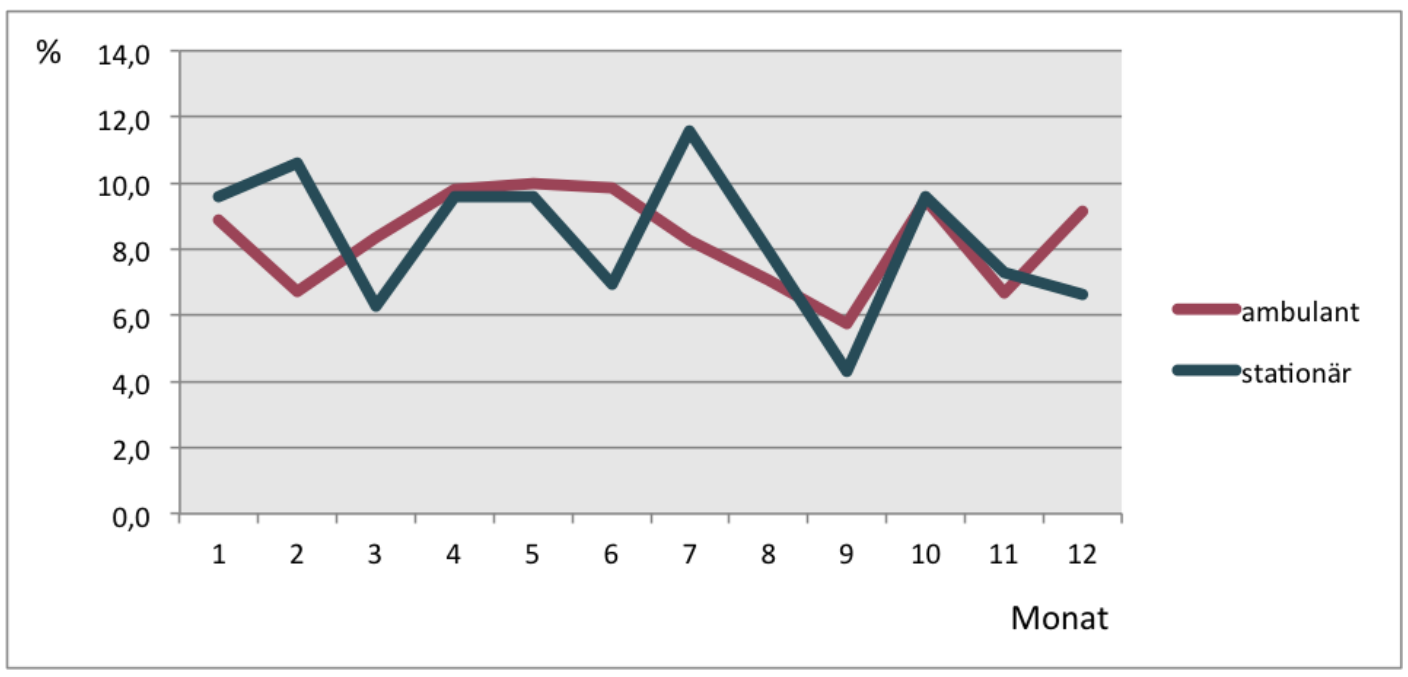

Abbildung 3.6: Häufigkeiten der Patientenkontakte (Anzahl in absoluten Zahlen) im Jahresverlauf (in Monaten)

\section{Wochenverlauf}

Das Erscheinen jedes Patienten wurde nicht nur im Jahres- (s. o.), sondern auch im Wochen- und Tagesverlauf dargestellt. Innerhalb der Woche zeichnete sich ein wellenförmiger Verlauf der ambulanten Patientenströme ab (vgl. Abb. 3.7). Von Montag bis Freitag stiegen die Besuche in den Abendstunden von 17 bis 22 Uhr an. In den Morgenstunden nahmen die Zahlen immer wieder deutlich ab (vgl. Abb. 3.7). Insgesamt kamen die meisten Patienten an den Wochenenden (Samstag und Sonntag). An diesen beiden Tagen suchten insgesamt 59,4\% bzw. 1.243 aller ambulanten Patienten die Klinik auf. Innerhalb der Wochenansicht waren die Stunden mit den höchsten Patientenzahlen samstags zwischen 11 und 12 Uhr (vgl. Abb. 3.7). 


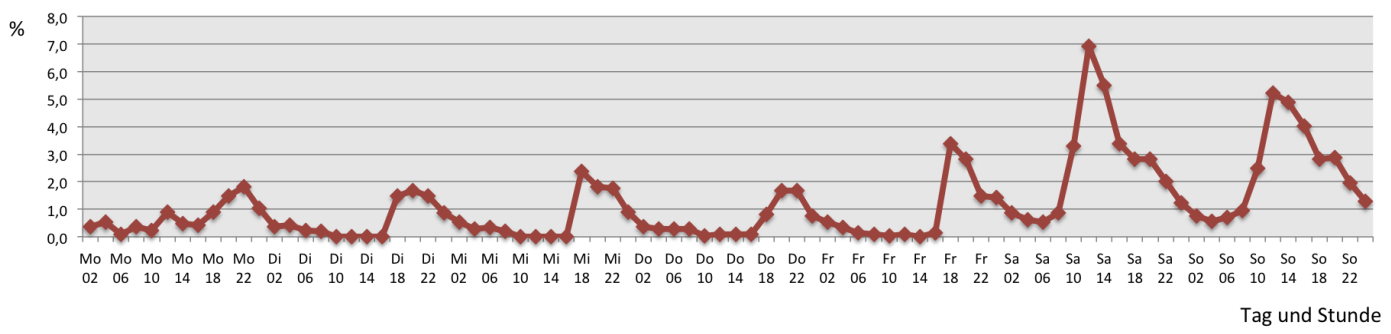

Abbildung 3.7: Häufigkeiten (in \%) der ambulanten Patientenkontakte innerhalb der Woche (in Tagen und Stunden). Anmerkung: Patientenkontakte zwischen zwischen 7:30 und 16 Uhr zwischen Montag und Freitag sind auf Notdienstbesuche an Feiertagen zurückzuführen.

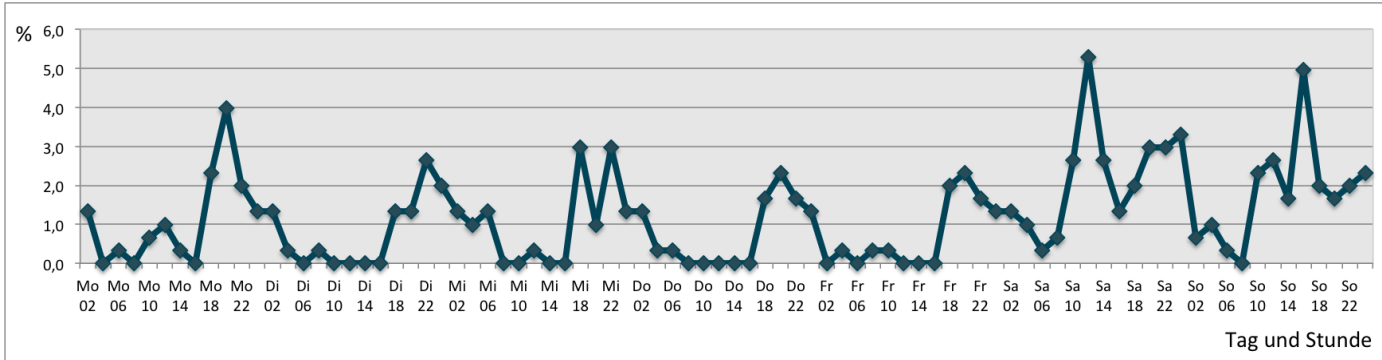

Abbildung 3.8: Häufigkeiten (in \%) der stationären Patientenkontakte innerhalb der Woche (in Tagen und Stunden). Anmerkung: Patientenkontakte zwischen zwischen 7:30 und 16 Uhr zwischen Montag und Freitag sind auf Notdienstbesuche an Feiertagen zurückzuführen. 
Bei den stationären Patienten war der Andrang in der Wochenansicht im Vergleich zu den ambulanten Patienten weniger periodisch (vgl. Abb. 3.7 f.). Die Wochenenden waren jedoch genauso wie bei den ambulanten Fällen am stärksten ausgelastet. Samstag und Sonntag wurden 48 \% (145 Patienten) aller stationären Notdienstpatienten behandelt (vgl. Abb 3.8). Die Stunden mit den höchsten Patientenzahlen waren samstags zwischen 11 und 12 Uhr (16 Patienten bzw. 5,3 \%) und sonntags zwischen 15 und 16 Uhr (15 Patienten bzw. $5 \%$ ) (vgl. Abb. 3.8). An den anderen Wochentagen (Montag bis Freitag) lag die Hauptstoßzeit innerhalb der Abendstunden zwischen 19 und 22 Uhr (vgl. Abb. 3.8). Innerhalb der Notdienstzeit kamen die wenigsten Patienten zwischen zwei und sieben Uhr morgens (vgl. Abb. 3.8).

\subsection{Spezielle Patientendaten}

Die Anamnese, Diagnostik und Therapie der häufigsten Diagnosen wurden weitergehend analysiert. Dabei wurde insbesondere darauf geachtet, ob Risikofaktoren und Komplikationen bei den untersuchten Patientenfällen festzustellen waren. Daneben wurde ermittelt, in welcher Häufigkeit Operationen notwendig waren und inwieweit die Patienten nach der Behandlung in der HNO-Klinik behandelt wurden.

\subsubsection{Risikofaktor Alter}

Das höchste durchschnittliche Alter wurde bei den ambulanten Patienten mit der Diagnose „Epistaxis“ (Mittelwert: 57 Jahre, Standardabweichung: 25 Jahre) ausgewertet, das geringste Durchschnittsalter in der Gruppe „akute Tonsillitis“ (Mittelwert: 26 Jahre, Standardabweichung: 10 Jahre) (vgl. Abb. 3.9, Tabelle 6.1 im Anang). Der Mittelwert mit 61 Jahren (Standardabweichung 23 Jahre) hat bei den stationären Patienten mit der Diagnose „Epistaxis“ den höchsten und bei den Patienten mit „chronischen Krankheiten der Tonsillen“ mit 9 Jahren (Standardabweichung: 9 Jahre) den niedrigsten Wert (vgl. Abb. 3.10 und Tabelle 6.2 im Anhang). 


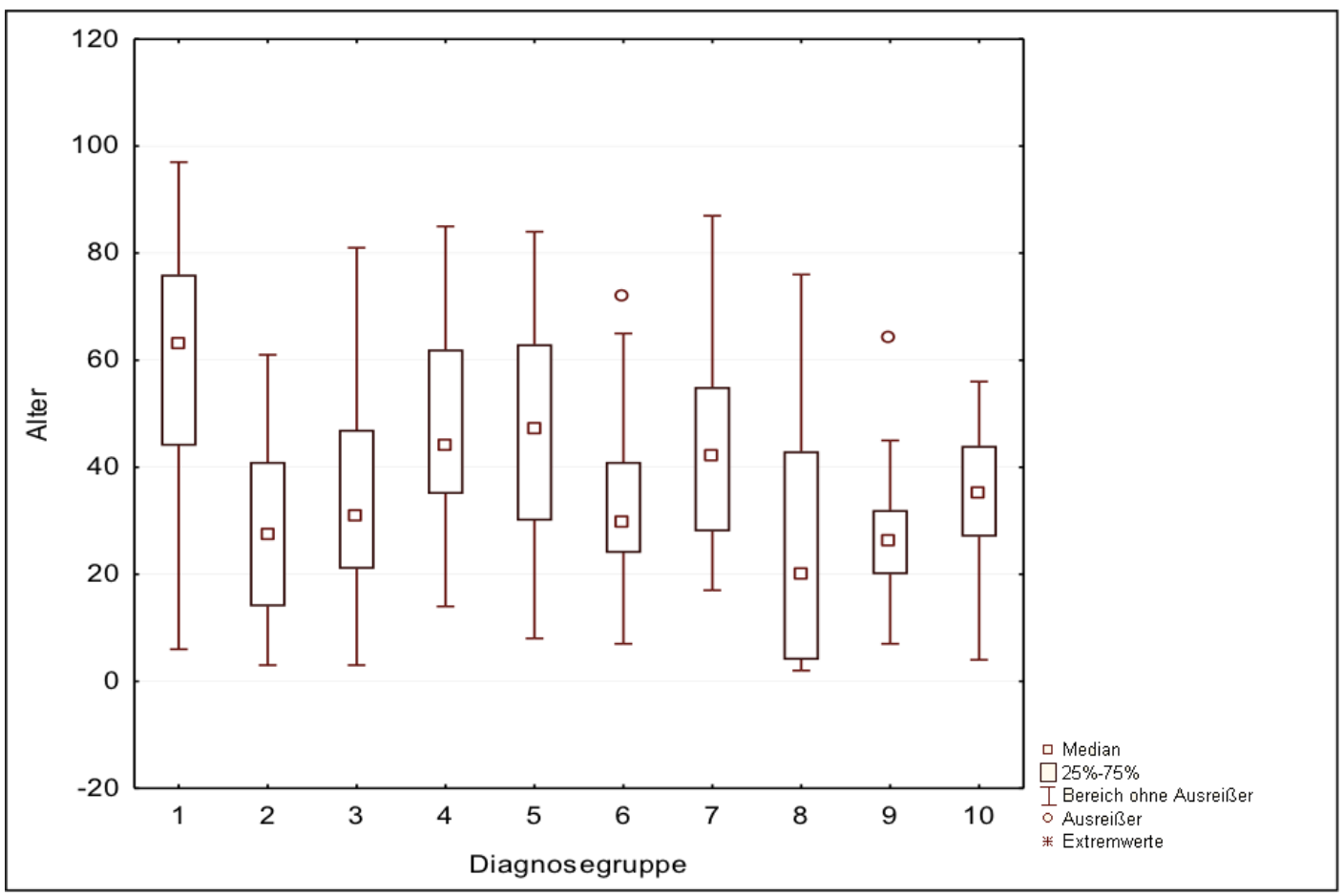

Abbildung 3.9: Vergleich des Alters (in Jahren) nach Diagnosegruppen der ambulanten Patienten; 1 = „Epistaxis“, 2 = „Eitrige Otitis media“, $3=$ „Otitis externa“, $4=$ „Zeruminalpfropf“, $5=$ „Hörverlust/Hörsturz", $6=$ „Akute Pharyngitis“, $7=$ „Tinnitus aurium“, $8=$ „Fremdkörper Atemwege“, $9=$ „Akute Tonsillitis“, 10 $=$ „chronische Sinusitis" 


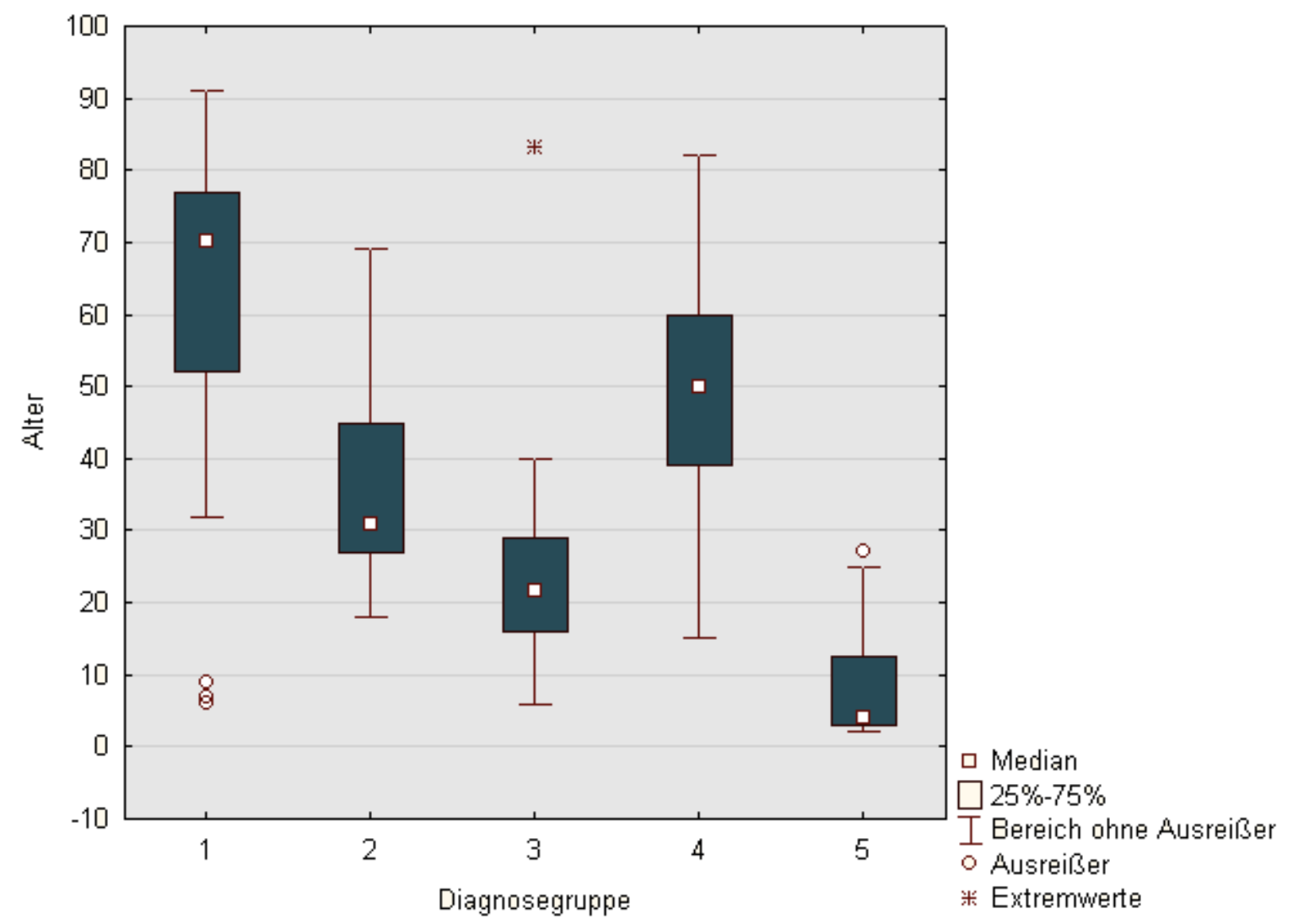

Abbildung 3.10: Vergleich des Alters (in Jahren) nach Diagnosegruppen der stationären Patienten; 1 = „Epistaxis“, $2=$ „Peritonsillarabszess“, $3=$ "Nachblutungen", $4=$ „Störungen der Vestibularfunktion“, $5=$ „chronische Krankheiten der Tonsillen"

\subsubsection{Risikofaktoren Allgemeinerkrankungen und Antikoagulanzien am Beispiel Epistaxis}

\section{Allgemeinerkrankungen}

Um möglicherweise Aussagen darüber zu erhalten, ob bestimmte Allgemeinerkrankungen bei Epistaxis-Patienten gehäuft gegeben waren, wurde die Anamnese dieser Patienten analysiert. Um mehr Übersichtlichkeit zu erhalten, wurden 
einzelne Erkrankungen in Gruppen zusammengefasst ${ }^{5}$.

Wenn bei den ambulanten Patienten die in Abb. 3.11 genannten Risikofaktoren (Herz-Kreislauf-, Lungen-, Nieren- und Leberkrankungen, Diabetes, Drogenkonsum und Nasen-OP) vorlagen, hatte der überwiegende Anteil nur einen dieser Risikofaktoren (40\%), während ein deutlich geringerer Anteil zwei Risikofaktoren (12\%) oder drei Risikofaktoren (2\%) aufwies. Bei $46 \%$ gab es keine Angaben zu den ausgewählten Risikofaktoren. Von den ausgewählten Risikofaktoren lagen die Herz-Kreislauf-Erkrankungen mit $44 \%$ deutlich im Fokus (vgl. Abb. 3.11).

Auch bei den stationären Patienten wurde der Risikofaktor Herz-KreislaufErkrankung am häufigsten genannt. In der Anamnese erwähnten 48,6 \% der Patienten, die aufgrund von „Epistaxis" den Notdienst aufgesucht hatten, an einer Herz-Kreislauf-Erkrankung zu leiden. Die anderen Risikofaktoren hatten Häufigkeiten unter $20 \%$ (vgl. Abb. 3.11).

Insgesamt gab es zu den 37 Patienten mit der Diagnose „Epistaxis“ 49 Angaben zu Risikofaktoren. Pro Patientenfall wurde am häufigsten ein Risikofaktor in der Anamnese genannt. Dies war bei 40,5 \% aller Patienten der Fall. Zwei Risikofaktoren wurden von 24,3\% und drei Risikofaktoren von 5,4 \% der Patienten genannt. Bei 29,7\% fanden sich hierzu keine Angaben (vgl. Abb. 3.11).

\footnotetext{
${ }^{5}$ 1. Herz-Kreislauf-Erkrankungen (HKE): Arterielle Hypertonie, Herzinsuffizienz, Vorhofflimmern, Vorhofinfarkt, Zustand nach Bypass-Operation, Zustand nach Stunt-Implantaten, Zustand nach Myokardinfarkt, Aortenklappenstenose, koronare Herzkrankheit, Kardiomyopathie, chronische arterielle Verschlusskrankheit und Herzarrhythmie 2. Lungenerkrankungen: Pneumonie, Zustand nach Lungenembolie, chronisch obstruktive Lungenerkrankung (COPD), Asthma, Lungenkarzinom. 3. Lebererkrankungen: Hepatitis A und C, Leberkarzinom. 4. Nierenerkrankungen: Terminale Niereninsuffizienz, bei vermerkter Dialysepflicht, Niereninsuffizienz und nicht näher in der Anamnese ausgeführte Nierenerkrankungen.
} 


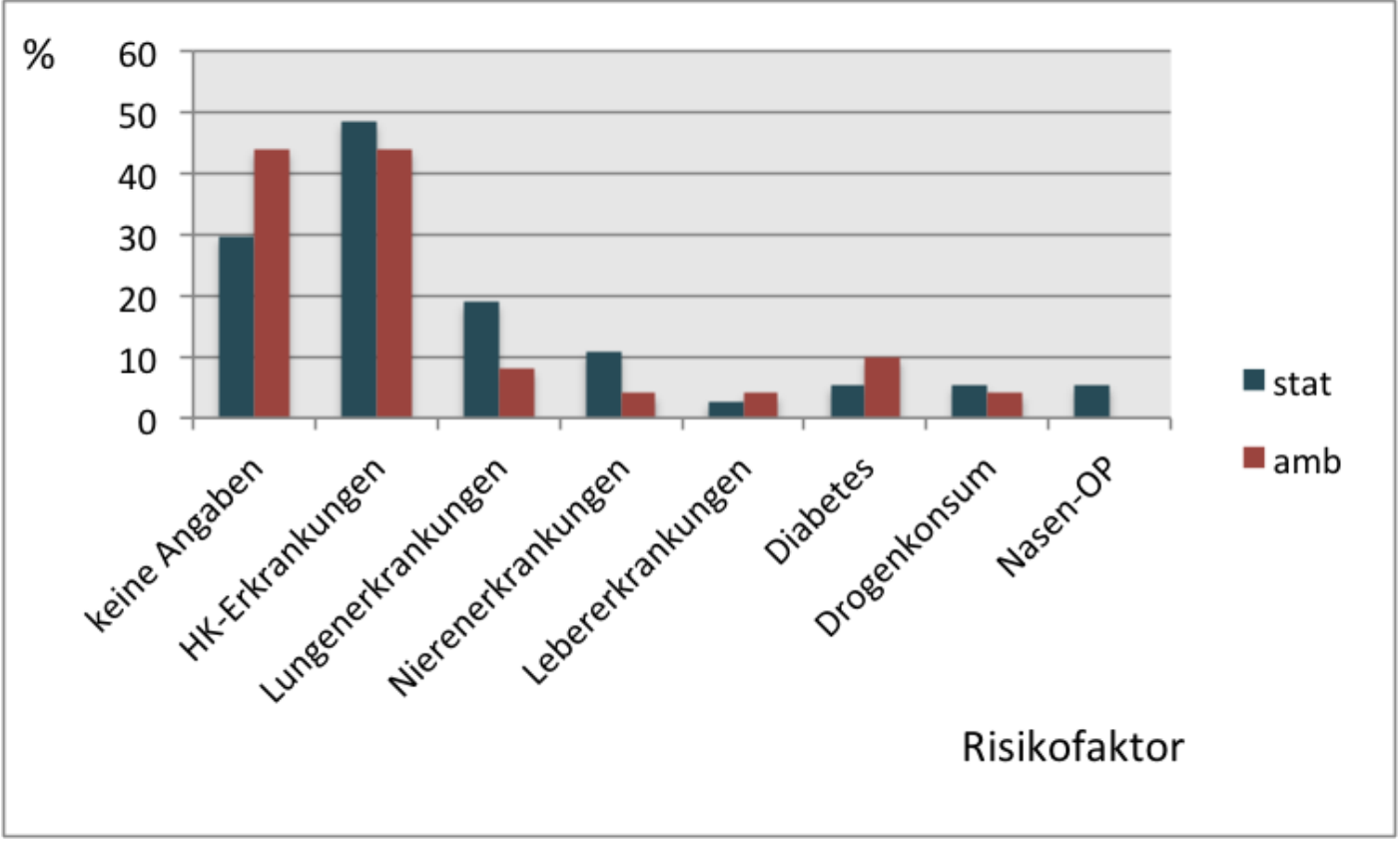

Abbildung 3.11: Relative Häufigkeiten (in \%) von Risikofaktoren bei stationären und ambulanten Patienten mit der Diagnose „Epistaxis“. Die relative Häufigkeit bezieht sich bei den stationären Patienten auf die Stichprobe von 37 Patientenfällen und bei den ambulanten Patienten auf eine Stichprobe von 50 Fällen mit der Diagnose „Epistaxis“. Es waren Mehrfachnennungen pro Fall möglich. 


\section{Antikoagulanzien}

Bei den Epistaxis-Patienten wurden die Angaben zu Antikoagulanzien in der Anamnese ebenfalls untersucht, um einen möglichen Zusammenhang zwischen der gehäuften Einnahme von Antikoagulanzien und dem Auftreten von Epistaxis herstellen zu können.

In der Anamnese wurde bei den ambulanten Patienten, die aufgrund von „Epistaxis“ therapiert wurden, Acetylsalicylsäure (ASS) als häufigstes Antikoagulans eingenommen, gefolgt von Phenprococoumon und Enoxaparin-Natrium (vgl. Tabelle 3.4). Bei den stationären Patienten war bei 19 der 37 (54 \%) die Einnahme von Antikoagulanzien angegeben. Die Einnahme von ASS war mit $37,8 \%$ am weitesten verbreitet (vgl. Tabelle 3.4).

Tabelle 3.4: Prozentuale Häufigkeiten der Einnahme von Antikoagulanzien bei den ambulanten und stationären Patienten mit der Diagnose „Epistaxis“. Die relativen Häufigkeiten beziehen sich auf die Stichprobe von 50 ambulanten Fällen und 37 stationären Fällen mit der Diagnose „Epistaxis“. Mehrfachnennungen waren möglich.

\begin{tabular}{|c||c|c|}
\hline Gruppe & amb. (\%) & stat. (\%) \\
\hline \hline ASS & 24 & 37,8 \\
\hline Phenprocoumon & 20 & 16,2 \\
\hline Enoxaparin-Natrium & 6 & 0 \\
\hline Clopidogrel & 4 & 2,7 \\
\hline Prasugrel & 2 & 0 \\
\hline unbekannte Antikoagulanzien & 26 & 0 \\
\hline keine Angaben & 42 & 45,9 \\
\hline
\end{tabular}




\subsubsection{Anzahl der Operationen bei den häufigsten Erkrankungen}

Exemplarisch wurde für die fünf häufigsten Diagnosen der stationären Patienten bestimmt, wie häufig eine „invasive“ Therapie vorgenommen wurde. Am häufigsten erfolgten operative Eingriffe in der Gruppe der „chronischen Krankheiten der Tonsillen"(vgl. Abb. 3.12). $92 \%$ der Patienten mit dieser Diagnose wurden operiert. Es bleibt jedoch zu berücksichtigen, dass es sich hierbei um sogenannte „Sonntagsaufnahmen“ gehandelt hat. Die Eingriffe waren geplant. Somit sind die häufigsten, ungeplanten Operationen bei Patienten mit „Nachblutungen“ vorgenommen worden. In der Gruppe mit der Diagnose „Störungen der Vestibularfunktion“ wurden keine operativen Maßnahmen durchgeführt (vgl. Abb. 3.12).

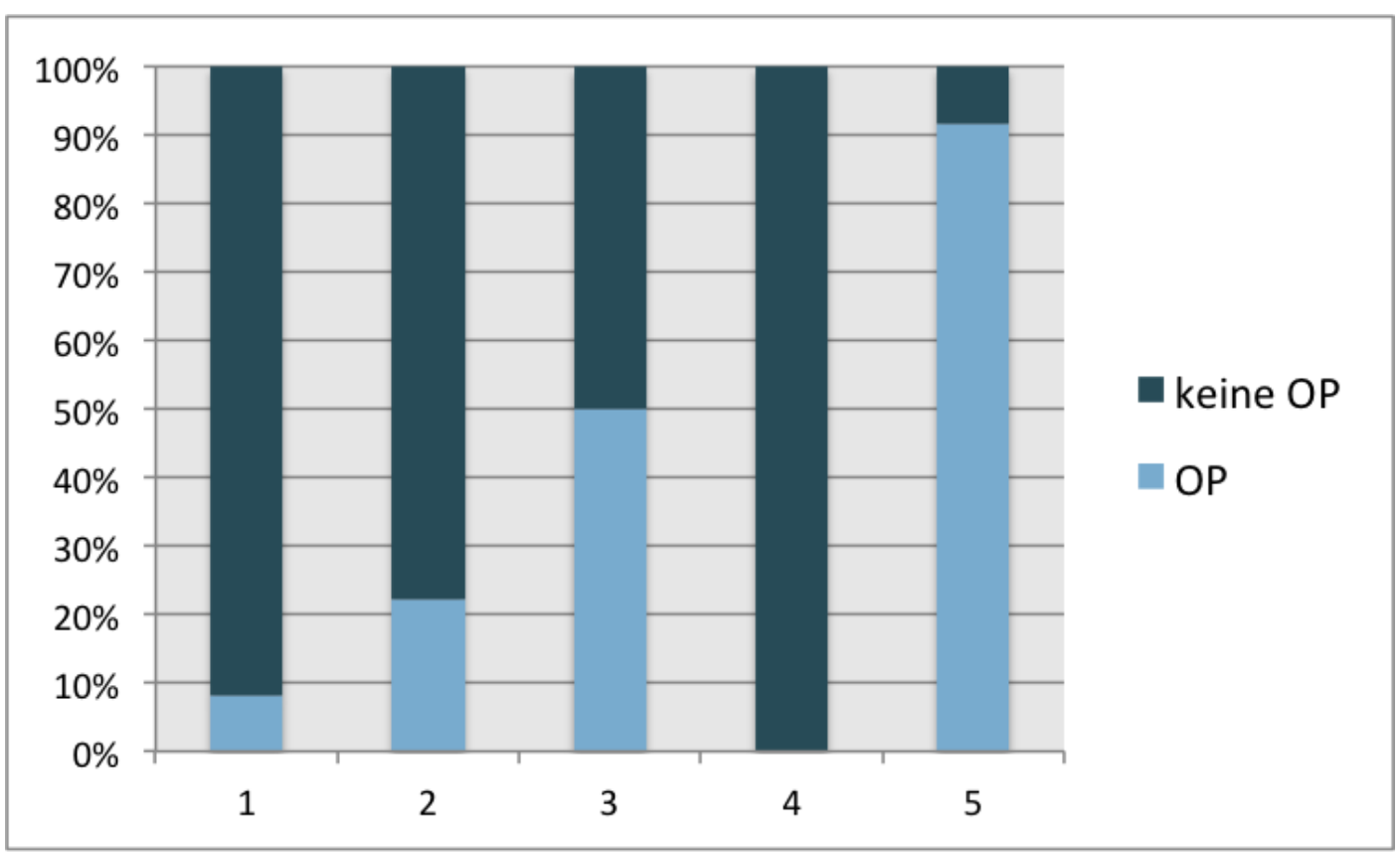

Abbildung 3.12: Operative Eingriffe nach Häufigkeiten (in \%) bei den stationären Patienten; 1 = „Epistaxis“, $2=$ „Peritonsillarabszess", $3=$ „Nachblutungen“", $4=$ „Störungen der Vestibularfunktion“, 5 = „chronische Erkrankungen der Tonsillen“ 


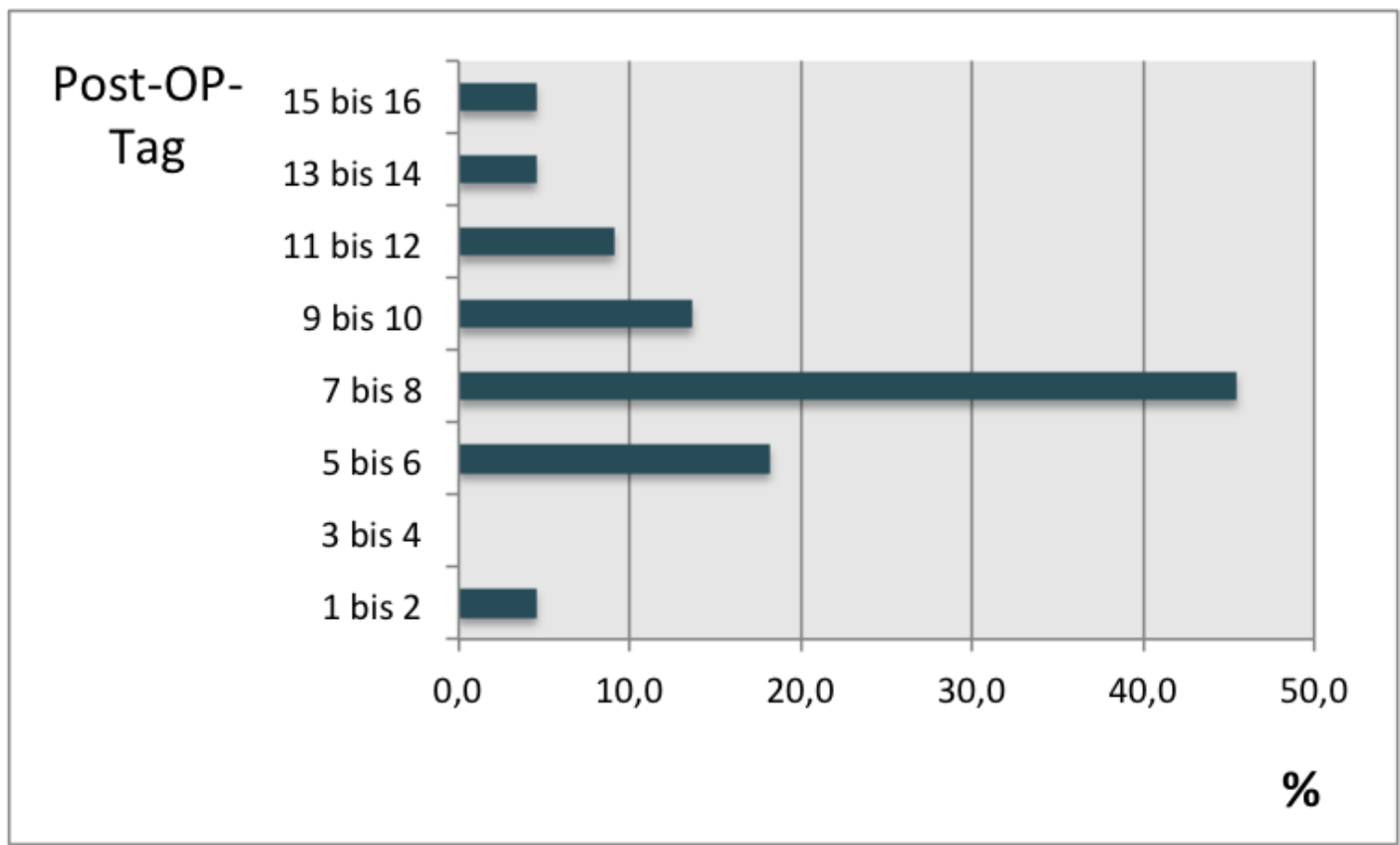

Abbildung 3.13: Verteilung von „Nachblutungen“ auf die jeweiligen postoperativen Tage (in \%). Die relative Häufigkeit bezieht sich auf die Stichprobe von 22 stationären Patientenfällen mit der Diagnose „Nachblutungen“.

\subsubsection{Besonderheiten der Diagnose „Nachblutung“}

Neben den Risikofaktoren, die eben exemplarisch für die Diagnose Epistaxis dargestellt wurden, stellten Nachblutungen (dritthäufigste Diagnosen bei den stationären Patienten, vgl. Tabelle 3.2) ebenfalls eine besondere Herausforderung im HNO-Notdienst dar. Nachblutungen traten in allen untersuchten Patientenfällen nach einer erfolgten Operation auf. Es wurde bestimmt, ob sich die Komplikation nach einer Operation in einem externen Krankenhaus oder im Universiätsklinikum ereignete. Weiterhin lag ein Augenmerk darauf, zu welchem postoperativen Zeitpunkt besonders häufig Nachblutungen vorkamen. Hierfür wurden die Angaben aus der Anamnese dokumentiert und ausgewertet.

In 54,5 \% der Fälle wurde vermerkt, dass die Patienten eine Vorbehandlung in einem anderem Krankenhaus erhalten hatten. In 45,5 \% war die Komplikation nach einer Behandlung in der HNO der UMG aufgetreten. Es wurde außerdem erhoben, welcher postoperative Tag zum Zeitpunkt des Notdienstbesuches vorlag (vgl. Abb 3.13). Bei 45,5\% war der Tag des Notdienst-Besuches am siebten oder achten postoperativen Tag (vgl. Abb. 3.13). Dies war der größte 
Teil der Patienten (vgl. Abb. 3.13). Bei 21 der 22 Fälle (95,5 \%) traten die Komplikationen nach Entfernung der Tonsillen auf (Tonsillektomie). In einem Fall $(4,5 \%)$ bestand die Nachblutung in einem Othämatom nach Exzision an der Ohrmuschel.

Bei der Hälfte der Patienten, die aufgrund einer Komplikation nach einem Eingriff stationär behandelt wurden, wurde eine Blutstillung in Vollnarkose durchgeführt. Die andere Hälfte der Patienten wurde konservativ versorgt. Von den 11 Fällen (50\%), die invasiv therapiert wurden, erfolgte die Operation bei 72,7 \% (8 Fälle) am Tag der stationären Aufnahme.

\subsubsection{Konsile}

Die Anzahl der Konsile wurden als ein Indikator dafür gewertet, wie groß die Interaktion des HNO-Notdienstes mit anderen Krankenhausabteilungen der UMG war. 194 Patienten (64,2 \%) wurden als erstes in der HNO-Klinik der UMG vorstellig und dort anschließend stationär aufgenommen. Der zweitgrößte Teil der stationären Behandlungsfälle umfasste 70 Patienten (23,2 \%). Diese Patienten wurden in der Zeit des Notdienstes von anderen Kliniken der UMG in die HNO-Klinik überwiesen und dort untersucht und ggf. dorthin verlegt. Die restlichen 38 Patienten (12,6 \%) wurden zunächst im Notdienst der HNO-Klinik untersucht und im Anschluss in andere Kliniken der UMG überwiesen.

Drei Kliniken wiesen eine besonders intensive Interaktion mit der HNO-Klinik auf. Dies sind in absteigender Reihenfolge die Kinderklinik, die Klinik für Neurologie und die Klinik für Innere Medizin (vgl. Tabelle 3.5). Zusammengefasst entfallen 74,1 \% aller konsiliarischen Interaktionen auf diese drei von sechzehn Kliniken (vgl. Tabelle 3.5). Die „chronischen Krankheiten der Tonsillen“ (acht Fälle) waren die häufigste Diagnose, weswegen andere Kliniken Patienten in die HNO-Klinik überwiesen. Die HNO hat am häufigsten ein Konsil aufgrund der Diagnose „Schwindel und Taumel“ (4 Fälle) angefordert. 
Tabelle 3.5: Überweisung (in absoluten und in relativen Häufigkeiten) von stationären Patienten zur und von der HNO-Klinik in den Notdienstzeiten. Gesamt Gr. 1 und Gr. 2: Summe der Interaktion aus Gr. 1 und Gr. 2. Gr. 1: Aufnahme durch die in der Zeile genannte Klinik mit anschließender Überweisung in die HNO-Klinik. Gr. 2: Erste Untersuchung in der HNO-Klinik mit anschließender Überweisung in die in der Zeile genannte Klinik der UMG.

\begin{tabular}{|c|c|c|c|c|c|c|}
\hline Kliniken & $\begin{array}{l}\text { Gesamt Gr. } 1 \\
\text { und Gr. } 2 \text { (abs.) }\end{array}$ & $\begin{array}{l}\text { Gesamt Gr. } 1 \\
\text { und Gr. } 2 \text { (in \%) }\end{array}$ & $\begin{array}{l}\text { Gr. } 1 \\
\text { (abs.) }\end{array}$ & $\begin{array}{l}\text { Gr. } 1 \\
\text { in } \%\end{array}$ & $\begin{array}{l}\text { Gr. } 2 \\
\text { (abs.) }\end{array}$ & $\begin{array}{l}\text { Gr. } 2 \\
\text { in } \%\end{array}$ \\
\hline Kinderklinik & 34 & 31,5 & 26 & 24,1 & 8 & 7,4 \\
\hline Neurologie & 24 & 22,2 & 11 & 10,2 & 13 & 12 \\
\hline Innere Medizin & 22 & 20,4 & 13 & 12 & 9 & 8,3 \\
\hline Kinderkardiologie & 7 & 6,5 & 6 & 5,5 & 1 & 0,9 \\
\hline Unfallchirurgie & 4 & 3,7 & 3 & 2,8 & 1 & 0,9 \\
\hline Frauenklinik & 3 & 2,8 & 3 & 2,8 & 0 & 0 \\
\hline $\mathrm{MKG}$ & 3 & 2,8 & 1 & 0,9 & 2 & 1,9 \\
\hline BUKH & 2 & 1,9 & 2 & 1,9 & 0 & 0 \\
\hline Psychiatrie & 2 & 1,9 & 2 & 1,9 & 0 & 0 \\
\hline Anästhesiologie & 1 & 0,9 & 0 & 0 & 1 & 0,9 \\
\hline Neurochirurgie & 1 & 0,9 & 1 & 0,9 & 0 & 0 \\
\hline Hautklinik & 1 & 0,9 & 0 & 0 & 1 & 0,9 \\
\hline Augenklinik & 1 & 0,9 & 0 & 0 & 1 & 0,9 \\
\hline THG-Chirurgie & 1 & 0,9 & 1 & 0,9 & 0 & 0 \\
\hline Allgemeinchirugie & 1 & 0,9 & 0 & 0 & 1 & 0,9 \\
\hline Orthopädie & 1 & 0,9 & 1 & 0,9 & 0 & 0 \\
\hline Summe & 108 & 100 & 70 & 64,8 & 38 & 35,2 \\
\hline
\end{tabular}




\subsubsection{Nachsorge}

Es war zudem von Interesse, inwiefern die Patienten nach der Behandlung im Notdienst weiterbehandelt wurden. Dafür wurde ausgewertet, wie viele der Patienten der häufigsten stationären und ambulanten Diagnosen innerhalb der ersten zwei Monate nach der Behandlung erneut ambulant oder stationär behandelt wurden. Insbesondere wurde ausgewertet, wie viele der Patienten innerhalb dieser zwei Monate erneut als Notfall behandelt wurden und wie viele der Patienten in den ersten zwölf Monaten nach dem Notdienst operiert wurden.

Innerhalb der ersten zwei Monate nach dem Notdienst-Besuch stellten sich 12,6 \% (63 der 500 Fälle) der ambulanten Patienten erneut in der HNO-Klinik vor. Die meisten Wiederkehrer wurden ambulant behandelt (88,9 \%), nur ein kleinerer Teil wurde stationär aufgenommen (11,1 \%). Am häufigsten wandten sich die Patienten aus der Gruppe „chronische Sinusitis“ erneut an die HNO-Klinik. Dies war bei 17 der 50 Fälle bzw. 34 \% der Fall (vgl. Abb. 3.14). Die wenigsten Wiederkehrer fanden sich bei den Patienten aus der Gruppe „Fremdkörper Atemwege“, „Pharyngitis“ und „Zeruminalpfropf“. Hier lag die Rate der Wiederkehrer bei nur zwei Prozent (vgl. Abb. 3.14). Sechs der wiederkehrenden Patienten waren erneut als Notfall in Behandlung (1,2 \%). Insgesamt wurden beim nächsten Patientenkontakt mit folgender stationärer Aufnahme nur wenige operative Eingriffe vorgenommen (0,8 \%). In der Gruppe „chronische Sinusitis“ wurde bei $6 \%$ der Fälle ein operativer Eingriff durchgeführt, in der Gruppe der „eitrigen Otitis media“ in $2 \%$ der Fälle. In allen anderen Gruppen wurden beim nächsten Patientenkontakt keine operativen Maßnahmen eingeleitet.

Bei den stationären Patienten stellten sich innerhalb der ersten zwei Monate nach der Notdienstbehandlung 25,8 \% (31 der 120 Fälle) erneut in der HNOKlinik vor. Bei dem ersten erneuten Kontakt wurden von diesen 31 Fällen 26 Fälle $(83,9 \%)$ ambulant und fünf Fälle $(16,1 \%)$ stationär therapiert Von den „Epistaxispatienten" kehrten acht Patienten (22,2 \%) innerhalb der ersten zwei Monate zurück, von den Patienten mit „Peritonsillarabszess“ neun Patienten (33,3 \%), von den Patienten mit „Nachblutungen“ (9,1 \%) zwei Patienten, von 
denen mit „Störungen der Vestibularfunktionen“ zehn Patienten (45,5\%) und von den Patienten mit „Chronischen Krankheiten der Tonsillen“ zwei Patienten $(16,7 \%)$. Am häufigsten wurden die Wiederkehrer operiert, die zuvor unter der Diagnose „Peritonsillarabszess“ im Notdienst aufgenommen worden waren. Dies traf auf 19 \% der Fälle mit „Peritonsillarabszess“ zu (vgl. Abb. 3.15). Ein Patient $(0,8 \%)$, der zuvor als stationärer Fall im HNO-Notdienst behandelt wurde, wurde innerhalb der ersten zwei Monate nach der Behandlung erneut zu den Zeiten des Notdienstes behandelt.

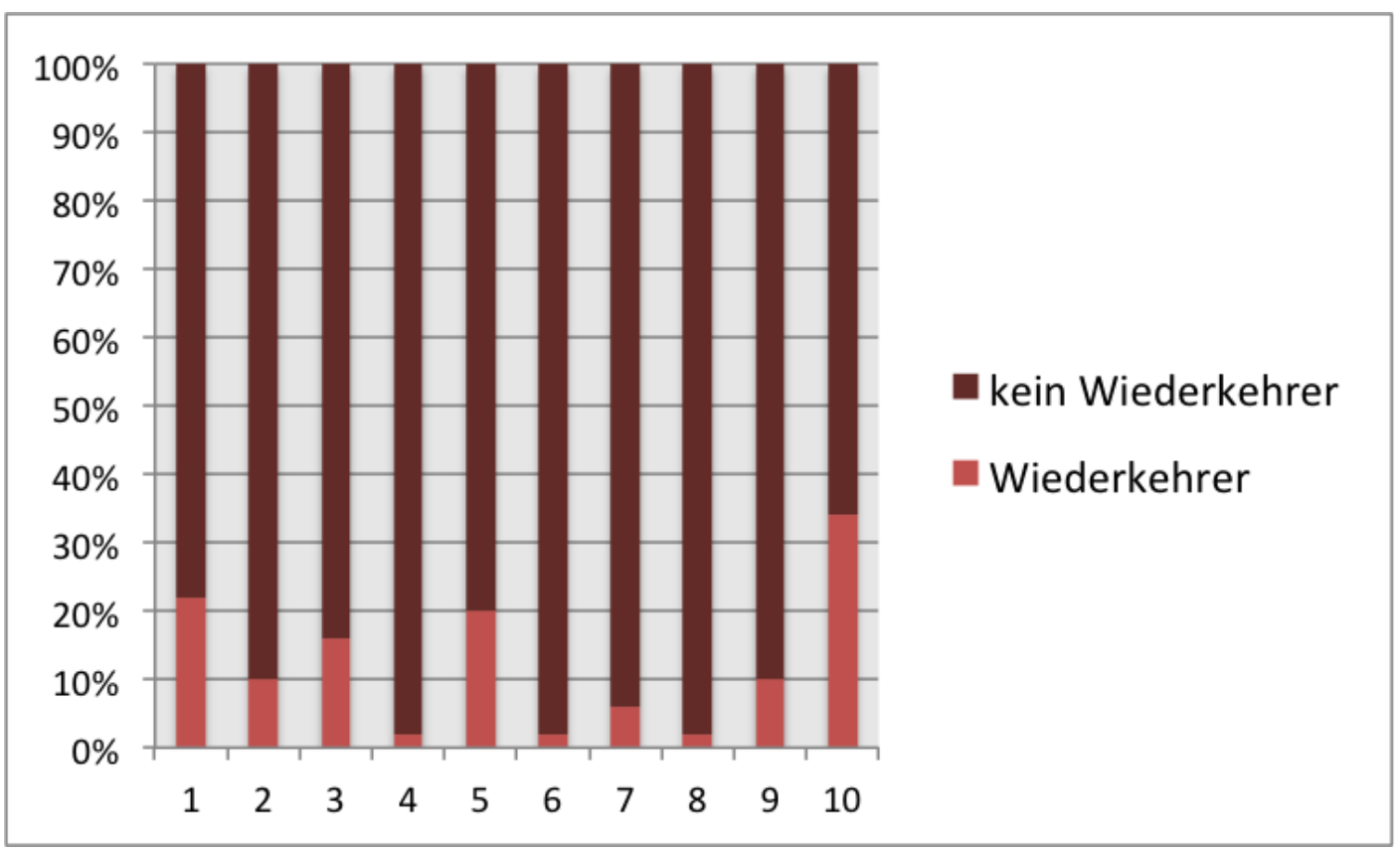

Abbildung 3.14: Wiederkehrer innerhalb der ersten zwei Monate nach NotdienstBesuch: Vergleich der Anzahl (in \%) nach den Diagnosegruppen der ambulanten Patienten; 1 = „Epistaxis“, $2=$ „Eitrige Otitis media“, $3=$ „Otitis externa“, $4=$ „Zeruminalpfropf", $5=$ „Hörverlust/Hörsturz", $6=$ „Akute Pharyngitis", $7=$ „Tinnitus aurium", $8=$ „Fremdkörper Atemwege“, $9=$ „Akute Tonsillitis", $10=$ „Chronische Sinusitis" 


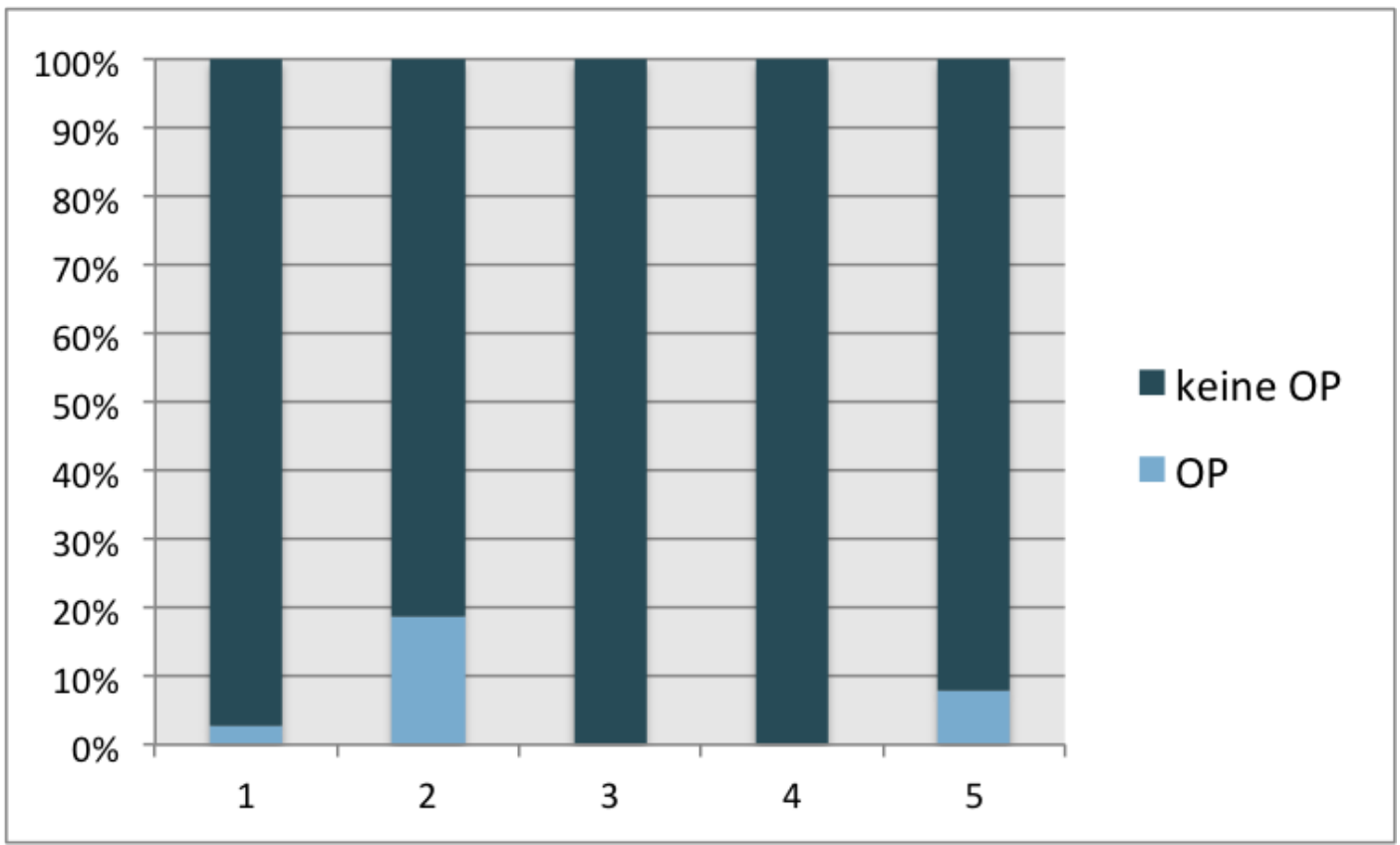

Abbildung 3.15: Wiederkehrer: Häufigkeiten (in \%) der operativen Eingriffe, die innerhalb der nächsten zwölf Monate nach stationärer Therapie vorgenommen wurden; $1=$ „Epistaxis“, $2=$ „Peritonsillarabszess“, $3=$ „Nachblutungen“, $4=$ „Störungen der Vestibularfunktion", 5 = „Chronische Erkrankungen der Tonsillen“" 


\section{Diskussion}

\section{1 Überblick}

In den bisherigen Kapiteln wurde die Stellung des HNO-Notdienstes im regionalen Versorgungsnetz dargestellt (Kapitel 1) sowie Methoden (Kapitel 2) und Ergebnisse (Kapitel 3) der Analyse vorgestellt. Damit wurde eine Grundlage gelegt, um im Folgenden die Funktion des HNO-Notdienstes zu diskutieren. Zunächst wird die Methodik der durchgeführten Analyse in Abschnitt 4.2 sowie die mittelfristige Analyse der Patientenzahlen und der Umfang der Analyse (vgl. Abschnitt 4.3) erörtert. Im Anschluss werden unter dem Oberthema „Generelle Patientendaten" Aufnahmeart, Diagnosen, Altersstruktur, Einzugsgebiet und Stoßzeiten diskutiert (vgl. Abschnitt 4.4). Im Teil „Spezielle Patientendaten“ folgen die Risikofaktoren und Komplikationen, Konsile und die Nachsorge (vgl. Abschnitt 4.5). Welche Schlussfolgerungen und Maßnahmen aus der Diskusssion abgeleitet werden können, wird im Abschnitt 4.6 behandelt.

\subsection{Methodik}

\subsubsection{Retrospektive Analyse}

Bei der vorliegenden Arbeit handelt es sich um eine retrospektive Analyse von Patienten, die im Jahr 2011 den HNO-Notdienst der Universitätsmedizin Göttingen aufgesucht haben. Retrospektive Analysen können Hinweise auf mögliche Ursachen geben, haben aber den Nachteil, dass keine kausalen Zusammenhänge nachgewiesen werden können (Weiß und Bauer, 2008). Außerdem ist das Datenmaterial teilweise unvollständig, was dazu führen kann, dass die wis- 
senschaftliche Aussagekraft begrenzt ist (Bertram und Farthmann, 1981). Während die Ergebnisse im Abschnitt „Generelle Patientendaten“ (vgl. Abschnitt 3.2) auf einem nahezu vollständigen Datenmaterial beruhen, wurden die Ergebnisse unter dem Abschnitt „Spezielle Patientendaten“ (vgl. Abschnitt 3.3) aus Patientenakten erhoben, bei denen nicht immer eine umfassende Dokumentation vorlag. Die retrospektive statistische Analyse von Patientendaten hat aber auch mehrere Vorteile. Die Studien sind zeitlich und umfangmäßig nicht begrenzt (Lorenz, 1981). Bertram und Farthmann sehen es als notwendig an, mit Hilfe retrospektiver Analysen Erkenntnisse zu gewinnen, da so eine kritische Auseinandersetzung mit dem eigenen Handeln stattfinde. Sie stellten eine gute klinische Entscheidungshilfe dar (Bertram und Farthmann, 1981).

In dieser Arbeit wurde eine retrospektive Analyse gewählt, weil eine umfassende und repräsentative Übersicht über die Patienten des HNO-Notdienstes das Ziel war und nicht, wie bei prospektiven Studien, eine gezielte Fragestellung im klinischen Ablauf untersucht werden sollte (Lorenz, 1981). Es könnte jedoch zukünftig interessant sein, auf Grundlage der Ergebnisse dieser Arbeit gezielt Parameter mit Hilfe prospektiver Analyse weitergehend zu untersuchen.

\subsubsection{Deskriptive und induktive Statistik}

Ein großer Teil der Daten wurde durch die sogenannte deskriptive Statistik ausgewertet. Die deskriptive Statistik erlaubt es, große Datenmengen übersichtlich darzustellen (Steland, 2010). Die Informationen werden durch die Verwendung von Kennzahlen und grafischen Darstellungen gebündelt (Hagl, 2008). Ein großer Nachteil der deskriptiven Statistik besteht jedoch darin, dass nur Aussagen zum Datensatz selbst gemacht werden können. Verallgemeinerungen sind nicht möglich. Steland [2010] wendet ein, dass keine stochastischen Modelle verwendet würden, so dass Ergebnisse nicht durch Fehlerwahrscheinlichkeiten abgesichert seien. 


\subsection{Mittelfristige Entwicklung der Patientenzahlen und Umfang der Analyse}

Der kontinuierliche Anstieg der Patientenzahlen von 2010 bis 2012 deutet darauf hin, dass der HNO-Notdienst an Bedeutung zunimmt. Es ist bei der Betrachtung der Werte jedoch zu berücksichtigen, dass ein Teil der Patienten nach unserer Definition nicht zu den „Notfallpatienten“ gezählt werden kann (vgl. Abschnitt 2.1 ff. ). Aber auch wenn nicht alle Patienten in diese Kategorie fallen, kann vermutet werden, dass der Trend steigender Patientenzahlen auch für unsere „Notfälle“ zutreffend war.

Das Phänomen steigender Patientenzahlen wird auch in der Literatur beschrieben. In einer Analyse der Notfallpatienten (aller Fachrichtungen) der Universität Magdeburg stellen die Autoren ebenfalls einen kontinuierlichen Anstieg der Zahlen in den letzten Jahren fest. Sie haben herausgearbeitet, dass nicht nur die Patienten, sondern auch das Verhalten der Einweiser/Pflegeheime, das Verhalten des Not- und Rettungsdienstes und des Krankenhauses eine Rolle spielen. Zudem sei der demographische Wandel von Bedeutung (Robra et al., 2013).

Es wäre interessant zu untersuchen, ob sich dieser Trend langfristig fortsetzt und eventuell eingeleitete Maßnahmen zur Steuerung der Patientenzahlen greifen. Daher wäre es sinnvoll, auch in Zukunft Patientenzahlen zu erheben und auszuwerten.

Der Umfang der Analyse lässt sich im Vergleich mit ähnlichen Studien bewerten. Die Stichprobe dieser Arbeit ist größer als bei Schlicht und Guntinas-Lichius [2012] (1.884 Patientenfälle), die ihre Ergebnisse in einer ähnlich großen Universitätsstadt erhoben haben und zudem aufgrund eines größer gewählten Zeitfensters mehr Patienten in die Untersuchung einschließen konnten ${ }^{6}$ (Schlicht und Guntinas-Lichius, 2012). Dies könnte dafür sprechen, dass dem HNO-Notdienst

\footnotetext{
${ }^{6}$ Es wurden alle Patienten eingeschlossen, die zwischen 16:00 Uhr und 7:30 Uhr von montags bis donnerstags und von freitags von 16:00 Uhr bis montags 7:30 Uhr den Notdienst der HNO-Klinik aufgesucht haben und in den elektronischen Verwaltungssystemen identifiziert werden konnten
} 
in Göttingen eine besondere Bedeutung in der regionalen Patientenversorgung zukommt. Insgesamt waren für das Jahr 20112.699 Notfälle registriert worden, von denen 2.396 in die Analyse einbezogen wurden. Die hohen Patientenzahlen lassen sich dadurch erklären, dass durch die Verfügung der kassenärztlichen Vereinigung nur die Universitätsmedizin Göttingen einen HNO-Notdienst außerhalb der Sprechstunden anbietet. Im Falle der Analyse von Schlicht et al. [2012] liegt eine andersartige Notdienstregelung vor. Möglicherweise sind die Patientenzahlen in dieser Analyse geringer, weil in Teilen Thüringens ein fachärztlicher Notdienst angeboten wird ${ }^{7}$.

Für die Patienten in Göttingen ist es möglicherweise einfacher, den HNONotdienst der Universitätsmedizin statt einen allgemeinen Bereitschaftsdienst aufzusuchen. Es entfällt die Suche nach der zuständigen Notfallpraxis und die ggf. langwierigere Anreise. Um die Suche für die Patienten zu erleichtern, wurde für den allgemeinen Bereitschaftsdienst im April 2012 eine zentrale Rufnummer eingeführt. Dies könnte dazu führen, dass in Zukunft mehr Patienten den allgemeinen Notdienst niedergelassener Ärzte wählen.

In dieser Arbeit richtete sich der Umfang der Analyse danach, wie viele Patienten in der Zeit des Notdienstes behandelt wurden und nicht danach, ob aus medizinischer Sicht ein Notfall vorlag. Eine Beschränkung auf die Fälle, die als medizinischer Notfall angesehen werden, wäre eine denkbare Alternative gewesen. Das Ziel dieser Arbeit war jedoch herauszufinden, mit welchen Fällen im HNO-Notdienst zu rechnen ist, unabhängig davon, wie schwerwiegend die einzelnen Behandlungsfälle einzustufen sind.

Schlussfolgerungen, ob die Patientenzahlen für eine hohe oder niedrige Belastung des medizinischen Personals sprechen, sind jedoch nur sehr eingegrenzt möglich. Der Fokus dieser Arbeit lag nicht auf betriebswirtschaftlich fokussierten Überlegungen.

\footnotetext{
${ }^{7}$ http://www.kv-thueringen.de/mitglieder/notdienst/2__Notdienstbereiche/HNO_ aerztlicher_Notdienst/HNO___rztlicher_Notdienst.pdf
} 


\subsection{Generelle Patientendaten}

\subsubsection{Aufnahmeart}

Es wurden sieben Mal so viele Patienten ambulant wie stationär therapiert (vgl. Abb. 3.2). Dieses extreme Verhältnis lässt vermuten, dass viele Patienten den Notdienst mit „leichten“ Erkrankungen aufgesucht haben. Ansonsten würde man davon ausgehen, dass ein höherer Anteil an stationären Patienten zu verzeichnen wäre (vgl. Abb. 3.2). In den Analysen der Universität Jena und der Universität Köln wurden im Verhältnis zu den ambulanten Patienten sogar noch weniger stationäre Patienten ermittelt (Schlicht und Guntinas-Lichius, 2012; Bolz, 2009). Dies kann damit zusammenhängen, dass in der vorliegenden Analyse alle stationären Fälle einbezogen wurden (also auch die zum Zeitpunkt der Behandlung bereits stationär aufgenommenen Patienten) und in den beiden anderen Analysen nur die neu aufgenommenen stationären Patienten.

Um weitergehende Bewertungen treffen zu können, empfiehlt es sich zu untersuchen, ob Patienten sich „selbst eingewiesen“ haben, überwiesen wurden oder mit einem Krankentransport eingeliefert wurden. Dieser Gesichtspunkt wurde in dieser Arbeit nicht weiter analysiert, könnte aber in zukünftigen Untersuchungen von Interesse sein.

Zusammenfassend zeigen die vorgelegten Ergebnisse, dass der Schwerpunkt im Notdienst auf der Versorgung „leichterer" Fälle liegt.

\subsubsection{Diagnosen}

Es wurden viele unterschiedliche Diagnosen für die untersuchten Patienten gestellt. Die zehn häufigsten Diagnosen bei den ambulanten Patienten deckten nur $54 \%$ aller ambulanten Diagnosen ab und die fünf häufigsten Diagnosen der stationären Patienten repräsentierten 32,9\% der stationären Diagnosen (vgl. Abschnitt 3.2.2). Angesichts der Vielfalt zu behandelnder Erkrankungen ist es daher schwierig, durch wenige Maßnahmen eine beträchtliche Verbesserung der Effizienz und der medizinischen Versorgung zu erzielen. Dennoch wird in dieser Arbeit versucht, für die häufigsten Diagnosen Trends abzulesen, die beispiels- 
weise für die gezielte Ausbildung des Personals genutzt werden könnten.

Die häufigsten Diagnosen der ambulanten Patienten verstärken den Eindruck, dass nicht immer eine sofortige medizinische Behandlung erforderlich gewesen wäre. Rivero et al. [2005] gewinnen aus ihren Ergebnissen die Erkenntnis, dass ein überwiegender Teil der Patienten den HNO-Notdienst mit „banalen“, unkomplizierten akuten und chronischen Erkrankungen konsultiert habe.

Die Ergebnisse aus den beiden anderen deutschen Studien fallen ähnlich aus (Bolz, 2009; Schlicht und Guntinas-Lichius, 2012). In der Untersuchung aus Jena fanden sich auf den vordersten Rängen dieselben Diagnosen in anderer Reihenfolge (Otitis media, Otitis externa und Epistaxis) (Schlicht und GuntinasLichius, 2012). Bolz führt an, dass Otitis media, Otitis externa und Tonsillitis die häufigsten drei Diagnosen bei den ambulanten Patienten waren (Epistaxis war auf dem fünften Rang) (Bolz, 2009). Auch in internationalen Arbeiten waren die häufigsten Diagnosen ähnlich ((Perez et al., 1995; Wheatley et al., 1999; Rivero et al., 2005). Die Diagnose „Epistaxis“ fand sich, anders als in dieser Arbeit, jedoch nur in einer Untersuchung ebenfalls auf dem ersten Platz (Rivero et al., 2005).

Warum ist gerade die Diagnose „Epistaxis“ so häufig im HNO-Notdienst der Universitätsmedizin Göttingen behandelt worden? Wie bereits erwähnt, wurde diese Diagnose auch in anderen Analysen besonders häufig erhoben. Dies spiegelt sich auch in der Lehrmeinung wieder (Probst et al., 2008). Probst et. al. [2008] beschreiben Nasenbluten als häufigsten HNO-Notfall, wobei lebensbedrohliche Blutungen eher selten seien. Dennoch sei es für den Patienten sehr unangenehm und könne ohne Therapie zu lebensbedrohlichen Blutverlusten führen (Nagel und Gürkov, 2009). Vor diesem Hintergrund erscheint es nicht sehr verwunderlich, dass ein Auftreten von Epistaxis für viele Menschen ein Besorgnis erregendes Ereignis darstellt und zum Aufsuchen eines Notdienstes veranlasst. Ursächlich können lokale (z. B. : Trauma durch Maniplulation und Fremdkörper) und systemische Ursachen (z. B.: Hämmorrhagische Diathesen) sein (Nagel und Gürkov, 2009). Es kann als Symptom vieler Erkrankungen auftreten und jede Altersgruppe kann davon betroffen sein (Folz et al., 2008). Auch 
meteorologische Einflüsse können zu einem erhöhten Auftreten von Epistaxis führen. Kanne [2007] berichtet davon, dass bei steigenden Luftdruckwerten und fallenden Temperaturen mehr Epistaxis-Fälle verzeichnet wurden.

Mehr Abweichungen hinsichtlich der häufigsten Diagnosen wurden in Analysen aus Schwellen- und Entwicklungländern dokumentiert. Dort wurden vor allem Fremdkörper besonders oft diagnostiziert (Saha et al., 2005; Kitcher et al., 2007). Die festgestellten Diagnosen könnten laut Kitcher et al. [2007] den sozioökonomischen Standard des jeweiligen Landes widerspiegeln, da in Entwicklungsländern ein hoher Anteil an vermeidbaren Diagnosen vorzufinden sei, der auf mangelnde Aufklärung zurückzuführen sei.

Ein Teil der Wissenschaftler fordert angesichts der hohen Anteile an „leichten“ Diagnosen daher, dass Allgemeinmediziner öfter als bisher leicht zu behandelne Fälle übernehmen sollten (Gallo et al., 2000; Kitcher et al., 2007).

Eine weitere denkbare Ursache für die hohe Anzahl an „leichteren“ Erkrankungen wäre, dass der Schweregrad der Erkrankungen von Patienten falsch eingeschätzt wurde. An der Queen's University kam man bei Untersuchungen des eigenen fachübergreifenden Notdienstes zu der Ansicht, dass die Erkrankungen in den meisten Fällen von den Patienten deutlich schwerer als von den behandelnden Ärzten eingestuft wurden (Kelly und Birtwhistle, 1993). In einer Analyse aus einem Hamburger Notfalldienstbezirk wurden nur sechs Prozent aller Erkrankungen als schwerwiegend bewertet (Staffa, 2007).

Diese falsche Einschätzung könnte darin begründet sein, dass auch banale Krankheiten Symptome schwerwiegender Krankheiten aufweisen können. Beispielsweise kann bei der Diagnose „Cerumen obturans“ u. a. eine plötzliche Hörbeeinträchtigung im Vordergrund stehen. Für viele Patienten ist eine plötzliche Hörbeeinträchtigung beunruhigend und schlecht einschätzbar, und auch für Fachärzte können dafür sowohl banalere als auch schwerwiegende Krankheiten in Betracht kommen. So kann die Ursache im äußeren und mittleren Ohr (Ohrschmalzpfropf, akuter Tubenmittelohrkatarrh, Barotrauma oder eine traumatische Trommelfellruptur) oder Innenohr liegen, aber auch durch funktionelle Probleme begründet sein (Hörsturz, Morbus Menière, akutes Schalltrauma oder 
eine psychogene Schwerhörigkeit) (Feldmann, 1973). Feldmann [1973] schreibt dazu, dass es sich nicht immer um Notfälle in dem Sinne handeln würde, dass nur durch ein sofortiges ärztliches Handeln ein schwerer Schaden abgewendet werden könne, sondern dass es z. T. auch banale Erkrankungen und Bagatellfälle seien. Die Symptomatik eines Bagatellfalles sei einer ernsten Erkrankung oft sehr ähnlich. Der Patient könne die Unterscheidung dabei nicht treffen. Die Versicherung, dass keine bedrohliche Erkrankung vorliege, sei eine ebenso verantwortungsvolle ärztliche Leistung wie die Abwendung einer akuten Gefahr durch eine spezifische Therapie (Feldmann, 1973).

Im Vergleich zu den diskutierten ambulanten Fällen zeigen die häufigsten Erkrankungen der stationären Patienten einen schwerwiegenderen Charakter ${ }^{8}$. Beispielsweise gilt der Peritonsillarabszess als eine potenziell lebensbedrohliche Erkrankung (Windfuhr und Remmert, 2005). Schmidt et al. schreiben, dass die Nachblutung die häufigste und gefährlichste Komplikation sei, die nach einer Tonsillektomie auftreten könne (Windfuhr und Remmert, 2005).

In der Literatur wurden nur in der Kölner Studie die häufigsten Diagnosen stationärer Notdienst-Patienten ausgewertet (Bolz, 2009). Dort waren der Peritonsillarabszess mit 32 Fällen, der Hörsturz mit 27 Fällen, Epistaxis mit 25 Fällen, bösartige Neubildungen mit 19 Fällen und die Faszialisparese mit 18 Fällen die fünf häufigsten Erkrankungen im Notdienst (Bolz, 2009). Damit stimmen zwei der Diagnosen aus den beiden Studien überein.

Die Analyse der stationären Diagnosen deutet darauf hin, dass Blutungen in Form von Epistaxis und Erkrankungen der Tonsillen und deren Komplikationen besonders häufig vorkamen. Als Konsequenz sollte das medizinische Personal speziell auf diese Fälle besonders vorbereitet sein.

\footnotetext{
${ }^{8}$ Bemerkung: Die fünfthäufigste Erkrankung bei den stationären Patienten, die „chronischen Krankheiten der Tonsillen“, waren geplante Sonntagsaufnahmen. Diese Diagnose kann daher nicht vollwertig in die Interpretation mit einbezogen werden.
} 


\subsubsection{Altersstruktur}

Die Auswertung der Altersstruktur gibt Hinweise, dass der Notdienst von einem Teil der ambulanten Patienten „inadäquat" genutzt wird. Es dominierten die Altersgruppen, die allgemein als wenig „krankheitsanfällig“ gelten und daher das Gesundheitssystem wenig in Anspruch nehmen (Rosenmayr, 2006). Am häufigsten waren die 21- bis 40-jährigen und am zweithäufigsten die 41- bis 60jährigen Patienten in ambulanter Behandlung. Auch wenn diese Altersgruppen in der Bevölkerungsstruktur Göttingens überrepräsentiert sind, ist dies bei der geringen Anfälligkeit dieser Jahrgänge bemerkenswert. Eine mögliche Erklärung wäre, dass diese Altersgruppen im besonderem Maße beruflichen und familiären Anforderungen ausgesetzt sind und daher vermehrt ärztliche Behandlung außerhalb der üblichen Sprechzeiten in Anspruch nehmen.

Andere Analysen kommen zu ähnlichen Ergebnissen. Von Bolz wurde beschrieben, dass bei den ambulanten Patienten der Altersgipfel zwischen 21 und 40 Jahren liege (Bolz, 2009). In Jena wurde der größte Anteil der Patienten im Alter zwischen 21 und 30 Jahren ausgemacht (Schlicht und Guntinas-Lichius, 2012).

Für den HNO-Notdienst der Universitätsmedizin Göttingen stellt sich die Frage, ob ein Andrang außerhalb der Sprechstunden erwünscht ist. Wäre dies nicht der Fall, sollten organisatorische Maßnahmen ergriffen werden, diesen Patientenstrom zu lenken. Kelly und Birtwhistle [1993] sind der Ansicht, dass Patienten besser aufgeklärt werden können, bei nicht dringlichen Fällen den Notdienst zu meiden, oder dazu bewogen werden sollten, den Allgemeinen Notdienst der niedergelassenen Ärzte aufzusuchen.

Die Altersstruktur der stationären Patienten zeigt hingegen, dass mit zunehmendem Lebensalter häufiger eine intensivere (in diesem Fall stationäre) Therapie notwendig ist. Das durchschnittliche Alter der stationären Patienten war mit 43,4 Jahren höher als bei den ambulanten Patienten mit 39,4 Jahren (vgl. Abschnitt 3.2.3). Laut Morrmann [2007] führt der demographische Wandel zudem dazu, dass es zu einer Verschiebung der Altersstruktur in den Krankenhäusern 
kommt und der Anteil älterer Patienten zunimmt. Damit steige auch der Anteil multimorbider und chronisch kranker Langzeitpatienten (Morrmann, 2007). Untersuchungen zur Ausgabenstruktur europäischer Gesundheitssysteme haben ergeben, dass u. a. die Altersgruppe der 61 bis 80 Jahre alten Patienten vermehrt medizinische Behandlung benötigt (Rosenmayr, 2006).

Die Gruppe der null bis fünf Jahre alten Patienten ist in der Altersstruktur der stationären Patienten ebenfalls auffällig stark vertreten (vgl. Abb. 3.3 und Abb. 3.10). Dies könnte damit begründet werden, dass das stärkere Auftreten dieser Jahrgänge auf die Sonntagsaufnahmen (Kinder mit „chronischen Krankheiten der Tonsillen“) zurückzuführen ist. Eine Aussage über eine besondere Anfälligkeit dieser Jahrgänge für den HNO-Notdienst kann folglich nicht getroffen werden. Dennoch bestätigen die häufigen Behandlungen von Kindern mit „chronischen Krankheiten der Tonsillen“ die Angaben aus der Literatur. Danach gehört die chronische Tonsillitis zu der Einzeldiagnose, die mit Abstand am häufigsten zu einem Krankenhausaufenthalt bei Kindern führt (Bitzer et al., 2009).

Aus den Ergebnissen der Altersstruktur und den häufigsten Diagnosen lässt sich folgern, dass ein besonderes Risiko für ältere Patienten mit der Diagnose Epistaxis besteht, stationär behandelt werden zu müssen. Ab 65 Jahren steigt insbesondere die Wahrscheinlichkeit, Komplikationen im Zusammenhang mit Epistaxis zu bekommen (Simmen und Heinz, 1998). Deshalb müssten sie häufig stationär therapiert werden. Dies folgerten sie aus ihrer Studie, die sich mit 365 Krankengeschichten von Epistaxis-Patienten befasste. Kotecha et al. [1996] kommen zu einem ähnlichen Ergebnis. Sie vermerken, dass Patienten mit Epistaxis vermehrt 60 Jahre und älter seien (Kotecha, 1996).

\subsubsection{Einzugsgebiet}

Die Auswertungen zum Einzugsgebiet zeigen, welche Bedeutung der Anfahrtsweg für ambulante und stationäre Patienten zum HNO-Notdienst hatte ${ }^{9}$. Der

\footnotetext{
${ }^{9}$ Bemerkung: Die Ergebnisse könnten dadurch leicht verzerrt sein, dass bei der Analyse nur der Erstwohnsitz der Patienten berücksichtigt wurde.
} 
HNO-Notdienst hatte für Patienten aus dem Stadtgebiet Göttingens eine besonders hohe Anziehungskraft. Es kamen 38 \% der ambulanten Patienten aus dem Stadtgebiet Göttingens, 32,6 \% aus dem direkten Umland (vgl. Abschnitt 3.2.4, Abb. 3.4). In Hinblick auf die Bevölkerungsdichte (vgl. Tabelle 3.3) erhält das Ergebnis noch mehr Aussagekraft. Für ambulante Patienten aus weiter entfernt liegenden Gebieten war der Anreiz, nach Göttingen zu fahren, deutlich niedriger. Eventuell warteten Patienten mit langem Anfahrtsweg häufiger auf den Wiederbeginn der Sprechzeiten (z. B.: bei einem niedergelassenen Allgemeinmediziner oder HNO-Arzt). Die Ergebnisse zeigen, dass die Inanspruchnahme des Notdienstes durch ambulante Patienten eher ein städtisches Phänomen ist. Es scheint so, dass Patienten sich eher medizinisch versorgen lassen, wenn der Anfahrtsweg kurz ist. Dies unterstreicht die These, dass unter den ambulanten Fällen eher „leichtere“ Fälle behandelt werden.

Die stationären Patienten kamen im Gegensatz zu den ambulanten Patienten am häufigsten aus dem direkten Umland der Stadt Göttingen. Von ihnen kamen 31,8\% aus dem direkten Umland (Postleitzahlengebiet 371XX, vgl. Abb. 3.4 f.) und nur 26,8 \% aus dem Stadtgebiet (vgl. Abschnitt 3.2.4, Abb. 3.5). Neben dem starken Patientenstrom aus dem unmittelbaren Umland hatte der HNO-Notdienst auch für die überregionale Versorgung eine größere Bedeutung. Möglicherweise hat ein höherer Leidensdruck der stationären Patienten dazu geführt, dass auch längere Wege zur medizinischen Versorgung in Kauf genommen wurden. Zudem kam eventuell nur noch fachärztliche Unterstützung in Frage. Weiterhin könnten demographische Faktoren eine Rolle gespielt haben. Möglicherweise ist der Anteil älterer Menschen im Umland etwas größer als in der Stadt Göttingen und daher der Anteil der Patienten, die stationär therapiert werden müssen, größer. Aber nicht alle weiter entfernt liegenden Gebiete sorgten für einen gleich hohen Patientenstrom (vgl. Abb. 3.5). Beispielsweise wies das Gebiet mit der Postleitzahl 375XX überregional die höchsten Patientenzahlen auf, obwohl für die Gebiete 376XX und 373XX höhere Bevölkerungsdichten vorlagen (vgl. Tabelle 3.3). Diese ungleichmäßige Verteilung könnte damit zusammenhängen, dass ein Teil der Patienten aus weiter entfernten Teilen Nieder- 
sachsens näherliegende Kliniken aufsuchten, die einen HNO-Notdienst anbieten. Die Stadt Hannover verfügt beispielsweise über einen eigenen HNO-Notdienst.

Es wäre interessant zu untersuchen, nach welchen Gesichtspunkten Patienten ein regionales Versorgungszentrum auswählen und inwieweit Patientenströme beeinflusst werden können.

\subsubsection{Stoßzeiten}

Über das Jahr gesehen wurde der HNO-Notdienst nicht gleichmäßig häufig von Notdienstpatienten aufgesucht. Im Mai, dem Monat mit den meisten ambulanten Patienten, kamen 86 Patienten mehr als im September, dem ruhigsten Monat (vgl. Abb. 3.6). Symvoulakis et al. [2006] verzeichneten ebenfalls die meisten Patienten im Frühjahr und führten dies auf das vermehrte Auftreten viraler Infektionen und allergischer Erkrankungen zurück (Symvoulakis et al., 2006). Dies konnte für diese Analyse nicht bestätigt werden. Im Vergleich zu den fünf häufigsten Erkrankungen des gesamten Jahres 2011 (vgl. Tabelle 3.1) zeigt der Monat Mai nur wenige Abweichungen. Lediglich die Diagnose „Frakturen des Schädels“ verdrängt die Diagnose „Krankheiten des Ohres“ von den ersten fünf Plätzen der Gesamtjahresstatistik. Die Schwankungen innerhalb des Jahres 2011 deuten an, dass die Arbeitsintensität für das medizinische Personal innerhalb des Jahres nicht gleichmäßig und damit wenig planbar ist.

Die Tages- und Wochenansicht zeigt, dass Stoßzeiten nicht nur im Jahresverlauf festzustellen sind (vgl. Abb. 3.7). Neben der „abweichenden“ Selbsteinschätzung der Patienten könnte eine weitere Ursache in den gestiegenen Anforderungen an die berufliche Verfügbarkeit der Patienten liegen. Diese führt zu mehr Arztbesuchen außerhalb der regulären Sprechstunden. Es kamen mehr Patienten am Samstag und Sonntag als an den übrigen Tagen, wobei in den Tagen von Montag bis Freitag die Abendstunden und an den Wochenenden die späten Morgenstunden stärker frequentiert waren (vgl. Abb. 3.7). Auch in der Studie aus Jena waren Samstage und Sonntage am stärksten fequentiert (Schlicht und Guntinas-Lichius, 2012). Bolz ist der Ansicht, dass gerade beruflich eingespannte Patienten die Arztbesuche in der regulären Dienstzeit mieden 
und auf die Notdienstzeiten der Klinik auswichen (Bolz, 2009). Dieses Verhalten beschrieben Mühlbauer und Kellerhoff in ihrem Buch „Zukunftsperspektiven in der Gesundheitswirtschaft“. Danach werden die regulären Öffnungszeiten von Patienten oftmals als ungünstig empfunden, sodass Wochenenden und abendliche Stunden dazu genutzt werden, Notaufnahmen aufzusuchen. „Der Begriff „Notaufnahme' wird fehlinterpretiert und als 24-Stunden-diagnostische-Serviceleistung unabhängig von der medizinisch indizierten Behandlungsdringlichkeit verstanden" (Muehlbauer und Kellerhoff, 2012, S. 84).

Ein weiterer Grund für den starken Andrang auf einen universitären Notdienst könnte sein, dass Patienten möglicherweise die medizinische Behandlung in einer Universitätsklinik als qualitativ besonders hochwertig einstufen.

Daneben könnte es eine Rolle spielen, dass die Wartezeiten auf einen Facharzttermin von vielen Patienten als zu lang empfunden werden und sie aus diesem Grund den HNO-Notdienst aufsuchen, um einen unmittelbaren Zugriff auf fachärztliche Versorgung zu erhalten. Cornelia Schmergal berichtet in der Zeitschrift „Der Spiegel“ über eine Berliner Notfallaufnahme und führt an, dass ein Teil der Patienten gezielt auf diese Art der Versorgung ausweichen, weil sie lange Wartezeiten auf einen Facharzttermin umgehen wollen (Schmergal, 2014). Dies könnte auch für Notdienstpatienten in unserer Untersuchung eine Rolle gespielt haben. Ob die Bestrebungen der Regierung, Wartezeiten auf einen Facharzttermin per Gesetz zu begrenzen, Veränderungen hervorrufen werden, wird sich zeigen.

Diese „falsche“ Inanspruchnahme kann dazu führen, dass wirklich dringliche Fälle nicht immer sofort medizinisch versorgt werden, weil „leichtere“ Fälle zu diesem Zeitpunkt das medizinische Personal binden. Des Weiteren kann dadurch eine Überlastung des medizinischen Personals und des Gesundheitssystems entstehen, die vermieden werden sollte (Gallo et al., 2000). Wie bereits erwähnt, müssen Patienten vermehrt darauf hingewiesen werden, dass die Behandlung im HNO-Notdienst nur für dringliche Fälle gedacht ist (Rivero et al., 2005). Ein anderer Ansatz wäre es, die Infrastruktur der Versorgung zu verändern. Der Ausbau von Notfallpraxen könnte helfen, die „leichteren“ 
Fälle zu versorgen (Walz, 2011). Mögliche Überlastungen könnten auch durch eine alternative Notdienstregelung erreicht werden. Länder wie beispielsweise Schleswig-Holstein und Thüringen bieten einen eingegrenzten HNO-Notdienst niedergelassener Ärzte an. Die Untersuchungen von Schlicht et al. [2012], die in Thüringen durchgeführt wurden, zeigen in Hinblick auf die Diagnosen und Stoßzeiten allerdings keine nennenswerten Unterschiede zu dieser Arbeit. Die alleinige Änderung der Notdienstregelung ist somit wahrscheinlich nicht zielführend. Nur in Kombination mit mehr Aufklärung kann dies erzielt werden.

Bei den stationären Patienten sind die Schwankungen etwas stärker ausgeprägt als bei den ambulanten Patienten. (vgl. Abb. 3.6). In der Tages- und Wochenansicht der stationären Patienten ist die Auslastung zwar ähnlich wie bei den ambulanten Patienten am Wochenende am höchsten, jedoch sind die Patientenbesuche innerhalb der Woche etwas gleichmäßiger auf den Notdienst verteilt als bei den ambulanten Patienten (vgl. Abb. 3.8). Da im Vergleich zu den ambulanten deutlich weniger stationäre Patienten betrachtet werden (statt 2094 nur 302 Patienten) ist der Vergleich und auch die Interpretation von saisonalen Schwankungen nur eingegrenzt möglich. Auch verzerren in dieser Untersuchung die Sonntagsaufnahmen das Bild der stationären Patienten etwas, so dass das eigentliche Notfallaufkommen am Wochenende in Wirklichkeit weniger stark einzustufen ist, als die Zahlen vermuten lassen.

Für die Stoßzeiten ist es wichtig, genügend Personal einzusetzen, um eine zügige Versorgung der Notdienst-Patienten zu gewährleisten (Rivero et al., 2005). Hier erweist sich der praktische Nutzen der Ergebnisse zu den Stoßzeiten des HNO-Notdienstes. Aussagen zu einer „Auslastung“ des HNO-Notdienstes im betriebswirtschaftlichen Sinne können und sollten mit dieser Arbeit nicht geleistet werden. In weiteren Studien könnte es interessant sein, dies durch Einbeziehung von Mitarbeiterzahlen und geleisteten Arbeitsstunden weitergehend zu untersuchen. 


\subsection{Spezielle Patientendaten}

Neben den oben genannten Kriterien wurden die Akten der Patienten mit den zehn häufigsten ambulanten und den fünf häufigsten stationären Diagnosen näher untersucht. Die wichtigsten Ergebnisse zu Risikofaktoren und Komplikationen werden im Anschluss diskutiert.

Ziel war es, mit diesem Teil der Analyse das medizinische Personal zu sensibilisieren und einen Beitrag für eine bessere Ausbildung zu leisten. Da insbesondere die stationären Patienten als kosten- und behandlungsintensiv gelten, lag der Schwerpunkt auf der Darstellung der stationären Patienten.

\subsubsection{Risikofaktor Alter}

Für die häufigsten ambulanten und stationären Diagnosen wurde das Durchschnittsalter bestimmt. Die Analyse zeigte, dass es für verschiedene Altersgruppen charakteristische Beschwerdebilder gab. Für die unterschiedlichen Aufnahmearten, ambulant und stationär, zeigten sich ebenfalls Unterschiede in der Altersstruktur.

Beispielsweise können die Beschwerdebilder „Eitrige Otitis media“ und „Otitis externa“ vergleichsweise jungen Patienten zugeordnet werden. Die Patienten mit der Diagnose „Eitrige Otitis media“ waren im Schnitt 28 Jahre alt und mit der Diagnose „Otitis externa“ 34 Jahre alt.

Die Diagnosen „Hörverlust/Hörsturz“ und „Zeruminalpfropf“" waren beispielsweise für das mittlere Alter typisch. Das Durchschnittsalter lag hier bei 48 bzw. 49 Jahren.

Besonders ältere Patienten fanden sich bei der Diagnose „Epistaxis“. Bei dieser Diagnose war es zusätzlich ausschlaggebend, ob sie ambulant oder stationär behandelt wurden. Ambulante Epistaxis-Patienten waren im Schnitt 57 Jahre alt und stationäre Patienten mit derselben Diagnose 61 Jahre alt (vgl. Tabelle $6.1 \mathrm{f}$.). Ein höheres Lebensalter stellt demnach ein Risiko dar, an Epistaxis zu erkranken. Je älter ein Patient ist, desto wahrscheinlicher wird eine stationäre Einweisung bei dieser Diagnose. Der höhere Anteil multimorbider Patienten 
wird u. a. für eine erhöhte Aufnahmewahrscheinlichkeit dieser Patientengruppe verantwortlich gewesen sein. Walker et al. verzeichneten in ihrer Analyse sogar ein Durchschnittsalter von 70 Jahren für die Epistaxis-Patienten (Walker et al., 2007). Allerdings errechnete sich dieser Wert nicht ausschließlich aus dem Alter der stationären Epistaxis-Patienten wie in dieser Analyse.

Aus den eben genannten Ergebnissen wird geschlossen, dass das Auftreten bestimmter Erkrankungen und das Alter der Patienten in einem Zusammenhang stehen. Sicherlich ist zu berücksichtigen, dass der Anteil jüngerer Patienten in der Stadt Göttingen überdurchschnittlich hoch ist. Damit erhalten die Schlussfolgerungen keine Allgemeingültigkeit, sondern gelten nur für diese Stichprobe.

\subsubsection{Risikofaktoren Allgemeinerkrankungen und Antikoagulanzien bei Epistaxis-Patienten}

Patienten mit der Diagnose „Epistaxis“ hatten nicht nur ein durchschnittlich höheres Lebensalter, sondern zeigten besonders häufig Allgemeinerkrankungen (vgl. Abschnitt 3.3.2). Bei der vorliegenden Arbeit waren dies vor allem Herzkreislauf-Erkrankungen, nämlich bei 48,6 Prozent der stationären und bei 44 Prozent der ambulanten Patienten (vgl. Abschnitt 3.3.2). HerzkreislaufErkrankungen werden in der Literatur als Risikofaktor für die Diagnose Epistaxis angesehen (Folz et al., 2008). Kanne [2007] stellte fest, dass insbesondere das Auftreten von Begleiterkrankungen bei Epistaxis-Patienten zur stationären Aufnahme geführt habe. Neben Herzkreislauferkrankungen wurden in dessen Analyse die Einnahme von Antikoagulanzien, regelmäßiger Tabak- und Alkoholkonsum, Blutgerinnungsstörungen, chronische Leber- und Nierenerkrankungen und Diabetes mellitus als häufigste Komorbiditäten angeführt (Kanne, 2007).

Dies wird durch die Ergebnisse dieser Untersuchung bekräftigt (vgl. Abschnitt 3.3.2).

Neben den Allgemeinerkrankungen wurde bei den ambulanten und stationären Patienten eine hohe Einnahme von Antikoagulanzien dokumentiert (vgl. Abschnitt 3.3.2). Besonders verbreitet war die Einnahme von ASS. $24 \%$ der ambulanten und 37,8 \% der stationären Patienten gaben in der Anamnese die 
Einnahme von ASS an (vgl. Tabelle 3.4). Die Einnahme von Antikoagulanzien zählt zu den wichtigsten ätiologischen Faktoren für die Diagnose Epistaxis (Kotecha, 1996; Dagan et al., 2012). Die hohen Zahlen von antikoagulierten Patienten lassen sich dadurch erklären, dass zunehmend mehr Patienten aufgrund internistischer Erkrankungen antikoaguliert werden müssen (Bermüller et al., 2014). Dies ist gerade bei älteren Patienten der Fall, die auch in unserer Untersuchung den größten Teil der Epistaxis-Patienten ausmachten (vgl. Abb. 3.10). Auch nach den Angaben des statistischen Bundesamtes steigt vor allem der Anteil der älteren Patienten, die eine stationäre Aufnahme benötigen, wenn sie unter Epistaxis leiden (Bermüller et al., 2014).

Die vorgenommene Analyse der Allgemeinerkrankungen und der Einnahme von Antikoagulanzien unterstreicht somit klinische Erfahrungen mit den jeweiligen Beschwerdebildern.

\subsubsection{Anzahl der Operationen unter den häufigsten Diagnosen}

Die Daten zu den Operationen zeigen, dass bei vier der häufigsten fünf Diagnosen zumindest zu einem Teil invasive Eingriffe vorgenommen wurden. Am häufigsten bei den „chronischen Krankheiten der Tonsillen“. Da es sich hierbei um die sogennanten „Sonntagsaufnahmen“ handelte, wurden Patienten mit der Diagnose „Nachblutungen“ am häufigsten ungeplant operativ versorgt. Da die Fallzahl der stationären Patienten bei den häufigsten fünf Diagnosen nicht sehr hoch war (12 bis 37 Patientenfälle), kann nur vermutet werden, dass „Störungen der Vestibularfunktionen“ eher konservativ und „Nachblutungen“ zu einem größeren Teil (hier zu 50\%) invasiv im HNO-Notdienst therapiert werden.

\subsubsection{Besonderheiten der Diagnose „Nachblutung“}

Die Ergebnisse zeigen, dass vor allem eine voraus gegangene Tonsillektomie die Hauptursache darstellt, wenn Patienten mit Nachblutungen den HNO-Notdienst aufsuchen. Bei 21 der 22 Fälle mit Nachblutungen wurde vorab eine Tonsillek- 
tomie durchgeführt. Die Nachblutung gilt als die häufigste und gefährlichste Komplikation nach Tonsillektomie (Schmidt et al., 1996). Die hohe Anzahl der Nachblutungen kann damit erklärt werden, dass die Tonsillektomie laut Statistischem Bundesamt zu den häufigsten Eingriffen in der Hals-, Nasen- und Ohrenheilkunde zählt ${ }^{10}$. In unserer Untersuchung kamen Patienten mit Nachblutungen vor allem am siebten oder achten postoperativen Tag. In der Literatur wird zwischen primären Nachblutungen in den ersten 24 Stunden nach Tonsillektomie und zwischen sekundären Nachblutungen unterschieden, die in der Regel zwischen dem fünften und zehnten postoperativen Tag auftreten (Windfuhr und Sesterhenn, 2001). Damit gehört der größte Teil der Nachblutungen aus dieser Untersuchung zu den sogenannten sekundären Nachblutungen. Sekundärblutungen gäben immer Anlass zu einer stationären Aufnahme. Bei wiederholten Nachblutungen könnten diese als Warnsignal für Massenblutungen interpretiert werden (Windfuhr und Sesterhenn, 2001).

Die Hälfte der Fälle mit Nachblutungen musste operativ versorgt werden. Dies zeigt, dass es sich um Notfälle handelt, deren Behandlung durch einen HNONotdienst dringend indiziert war. Die invasiven Eingriffe erfolgten zu 72,7\% am Tag der stationären Aufnahme. Dies unterstreicht den akuten Therapiebedarf. Die Angaben aus der Literatur und auch die Ergebnisse dieser Analyse zeigen, dass die Behandlung von Nachblutungen grundsätzlich stationär zu erfolgen hat (Windfuhr und Sesterhenn, 2001). Eine längere Beobachtungszeit bei auftretenden Nachblutungen ist zu empfehlen. Dennoch muss nach Windfuhr und Sesterhenn [2001] immer abgewogen werden zwischen einer medizinischen Notwendigkeit und der Finanzierbarkeit einer längeren stationären Behandlung nach Tonsillektomie. In ihrer Analyse kam es bei 5474 vorgenommenen Tonsillektomien lediglich zu 145 Nachblutungen (Windfuhr und Sesterhenn, 2001).

Weiterhin sollte eine sorgfältige Gerinnungsanamnese standardmäßig erhoben werden (Scheckenbach et al., 2008). Eine präoperative Bestimmung von Routineparametern der Gerinnung ist jedoch nicht erforderlich (Scheckenbach

\footnotetext{
${ }^{10}$ vgl. https://www.destatis.de/DE/ZahlenFakten/GesellschaftStaat/Gesundheit/ Krankenhaeuser/Tabellen/HaeufigstenOPSFrauen.html
} 
et al., 2008). Aus Scheckenbachs [2008] Sicht decken diese nicht sicher korrekturbedürftige Gerinnungsstörungen auf und geben keine sichere Vorhersage darüber, ob es zu einer Blutungskomplikation kommen wird (Scheckenbach et al., 2008).

\subsubsection{Konsile}

Die Ergebnisse zu den Konsilen des HNO-Notdienstes zeigen, dass dieser HNONotdienst mit sehr vielen Krankenhausabteilungen der UMG zusammengearbeitet hat. Die Arbeit mit drei Abteilungen war besonders intensiv (vgl. Tabelle 3.5). Dies waren die Innere Medizin, die Pädiatrie und die Neurologie (vgl. Tabelle 3.5). Symvoulakis et al. [2006] bestätigen in ihrer Analyse die Ergebnisse dieser Arbeit. Die häufigsten drei Konsile fanden in seiner Studie mit absteigender Häufigkeit mit der Inneren Medizin, der Klinik Pädiatrie und der Klinik Neurologie statt (Symvoulakis et al., 2006). In der Analyse am Universitätsklinikum in Köln wurde genauso wie in dieser Untersuchung bei einer Schwindelsymptomatik am häufigsten ein Konsil angefordert (Bolz, 2009). Die Aussagekraft ist aber angesichts der geringen Fallzahl (vier Fälle) in der vorliegenden Arbeit begrenzt.

Auffällig ist zudem, dass viele Kinder nach der Aufnahme in der Kinderklinik im weiteren Verlauf in die HNO überwiesen wurden (vgl. Tabelle 3.5). Dies könnte bedeuten, dass die Kinderklinik in besonderem Umfang Überweisungen zu anderen Fachrichtungen vornimmt. Weiterhin zeigen die Ergebnisse, dass der HNO-Notdienst weniger zu anderen Fachrichtungen überwiesen hat als andere Fachrichtungen in die HNO überwiesen haben (vgl. Tabelle 3.5). Im Gegensatz zur Kinderklinik scheint die HNO-Klinik weniger eine „Verteilungsfunktion“ zu übernehmen, was damit zu erklärten ist, dass eine Kinderklinik zwangsläufig breiter diagnostisch und therapeutisch tätig sein muss.

Es scheint also wichtig zu sein, dass das Personal der Pädiatrie im Erkennen und Behandeln von Erkrankungen der Hals-Nasen-Ohrenheilkunde gut ausgebildet wird. Möglicherweise könnte eine intensivere Zusammenarbeit mit den Krankenhausabteilungen der Inneren Medizin, der Pädiatrie und der Neurolo- 
gie förderlich sein im Sinne der Optimierung der notwendigen Funktionsabläufe.

\subsubsection{Nachsorge}

Der Nachsorgebedarf wurde zum einem daran gemessen, wie hoch der Anteil der Patienten war, die innerhalb der ersten zwei Monate erneut in ambulanter oder stationärer Behandlung waren (und wie viele davon als Notfall), und zum anderen, wie hoch der Anteil der Patienten war, der innerhalb der ersten zwölf Monate nach dem Notdienstbesuch operiert wurde (vgl. Abschnitt 3.3.6).

Eine weitere konservative Behandlung und insbesondere eine invasive Therapie schien stark von der bisherigen Aufnahmeart der Patienten abhängig gewesen zu sein. Im Vergleich der ambulanten zur stationären Nachsorge zeigte sich, dass in den ersten zwei Monaten nach der Notdienst-Behandlung etwa doppelt so viele stationäre Patienten $(25,8 \%$ ) wie ambulante Patienten $(12,6 \%)$ erneut die HNO-Klinik aufsuchten. Weiterhin wurden deutlich mehr stationäre (5,8 \%) als ambulante Patienten (0,8 \%) im ersten Jahr nach der Notdienst-Behandlung operiert. Dabei war es stark davon abhängig, mit welcher Diagnose die Patienten ursprünglich im Notdienst behandelt worden waren. Bei den stationären Patienten wurden besonders häufig Patienten mit der Diagnose „Peritonsillarabszess“ (19\%) operiert, bei den ambulanten Patienten solche mit der Diagnose „chronische Sinusitis“(6 \%). In der Analyse von Lasisi et al. [2007] wurde ebenfalls die chronische Sinusitis als Diagnose beschrieben, die im Notdienst häufiger aufgrund von Komplikationen behandelt werden musste (Lasisi et al., 2007). Die Anzahl der Patienten, die erneut als Notfall in der HNO-Klinik behandelt wurden, war gering. Nur 1,2\% der ambulanten und 0,8\% der stationären Patienten waren innerhalb der ersten zwei Monate zu den Zeiten des Notdienstes in Behandlung. Dies könnte für eine geringe Komplikationsrate der Behandlungen im Notdienst sprechen. Wie viele Patienten langfristig weitere medizinische Versorgung benötigten und wie viele Patienten bei niedergelassen Ärzten weiter therapiert werden mussten, zeigen die Ergebnisse nicht. Für zukünftige Untersuchungen könnte dies von Interesse sein. Aber auch wenn die Weiterbehandlung bei den niedergelassenen Ärzten nicht gesondert festgehalten wurde, kann nach 
unseren Zahlen vermutet werden, dass der größte Teil der Behandlungen im Notdienst entweder abgeschlossen werden konnte oder zumindest von niedergelassen Kollegen im Anschluss ausreichend betreut werden konnte.

\subsection{Schlussfolgerungen}

Der HNO-Notdienst übernimmt einen umfangreicheren Teil der medizinischen Versorgung, als ihm von der KVN zugedacht ist. Er wird nicht nur mit steigenden Patientenzahlen, sondern auch mit vielen „banalen“ Erkrankungen kon-

frontiert. Die Ärzte im HNO-Notdienst werden mit einem breiten Spektrum an Erkrankungen konfrontiert, wobei in dieser Stichprobe die Mehrheit der Fälle ambulant und konservativ therapiert werden konnte. Damit bestätigen diese Ergebnisse vorangegangene Untersuchungen.

Welche Maßnahmen könnten angesichts dieser Ergebnisse ergriffen werden? Zum einen gibt es die Möglichkeit, die steigenden Patientenzahlen zu regulieren. Zum anderen könnte die Qualität der medizinischen Versorgung gesteigert werden.

\subsubsection{Regulierung der Patientenzahl}

Es wurden im Laufe der Diskussion vielfältige Ursachen für den Anstieg der Zahlen verantwortlich gemacht. Neben der falschen Selbsteinschätzung und einer veränderten Anspruchshaltung der Patienten kommen auch andere Faktoren in Betracht. Man sollte bei der Planung des Notdienstes festlegen, welche Rolle er im regionalen Versorgungsnetz anstrebt. Falls die Patientenzahl für den HNO-Notdienst zu groß ist, könnte er durch bessere Aufklärung erreichen, dass eine medizinische Versorgung außerhalb der Sprechstunden weniger in Anspruch genommen wird. Ein anderer Ansatz wäre es, die Primärversorgung zu verbessern. Es sollte überlegt werden, wo Praxen überlastet sind und was dagegen unternommen werden kann. Die medizinische Versorgung muss an die jeweiligen Gegebenheiten der Regionen angepasst werden (Robra et al., 2013). Ein weiteres Mittel zur Regulierung der Patientenzahlen, wäre die Einrichtung 
sogenannter kliniksnaher „Notfallpraxen“, in denen vor allem die Behandlung „leichterer“ Fälle vorgenommen werden könnte (Fischer, 2009).

Zudem könnten Patienten vom medizinischen Personal bei nicht dringlichen Behandlungen angewiesen werden, bis zu den regulären Sprechzeiten zu warten. Dies ist jedoch aufgrund rechtlicher und ethischer Bedenken nicht umsetzbar. Eine Sortierung der vorhanden Patienten ist jedoch sinnvoll und wird bereits andernorts praktiziert. Beim „Manchester Triage“ handelt es sich um ein Auswahlsystem bei dem dringliche und weniger dringliche Fälle durch unterschiedliche Farben gekennzeichnet werden, um eine übersichtliche Behandlungsreihenfolge zu schaffen (Schmergal, 2014).

Zusätzlich könnte versucht werden, die Erreichbarkeit des allgemeinen Bereitschaftsdienstes so zu verbessern, dass es für Patienten attraktiver ist, diesen aufzusuchen. Dies könnte beispielsweise durch eine zentrale Notfallpraxis geleistet werden.

In dieser Arbeit wurde davon ausgegangen, dass die Zunahme der Patienten im HNO-Notdienst nicht prinzipiell erwünscht ist. Möglicherweise werden die steigenden Patientenzahlen jedoch nicht als Belastung empfunden, sondern sogar angestrebt. In diesem Falle stellt sich ein möglicher Maßnahmenkatalog anders dar. Die Frage ist dann, wie Patienten beeinflusst werden können, den HNO-Notdienst als Versorgung zu wählen. Gezielte Werbung in niedergelassen Praxen für eine Versorgung außerhalb der Sprechzeiten könnte dabei helfen. Eine Intensivierung der Zusammenarbeit mit den niedergelassenen Kollegen, Pflege- und Ärztediensten wäre dann wünschenswert.

\subsubsection{Steigerung der Qualität}

Um Aussagen darüber treffen zu können, wie die Qualität der medizinischen Versorgung gesteigert werden kann, wurden die häufigsten Diagnosen der ambulanten und stationären Patienten hinsichtlich der Anamnese, Diagnostik und Therapie nach möglichen Risikofaktoren und Komplikationen untersucht.

Die Ergebnisse zeigten, dass das medizinische Personal besonders für ältere, multimorbide Patienten sensibilisiert sein sollte. Auch die Einnahme von Me- 
dikamenten spielt für die Arbeit im HNO-Notdienst eine wichtige Rolle. Die Anamnese der Patienten ist somit als zentral einzustufen und sollte stets mit der nötigen Sorgfalt erhoben werden. Die Notwendigkeit einer stationären Therapie erhöht sich bei Epistaxispatienten insbesondere wenn Allgeimeinerkrankungen, die Einnahme von Antikoagulanzien und ein erhöhtes Lebensalter kombiniert vorliegen. Eine besondere Nachsorge und zeitlich umfassende Therapie benötigen Patienten nach erfolgter Tonsillektomie. Eine frühzeitige Entlassung kann dazu führen, dass Patienten aufgrund von Nachblutungen erneut aufgenommen werden müssen. Auch unter dem derzeit vorhanden Kostendruck darf die Qualität der medizinischen Versorgung nicht leiden.

\subsection{Ausblick}

Es empfiehlt sich, die unterschiedlichen Notdienstsysteme der einzelnen Länder noch intensiver als in dieser Arbeit miteinander zu vergleichen. Denn es stellt sich die Frage, wie die Konsequenzen ganz genau aussehen, wenn ein HNOBereitschaftsdienst niedergelassener Ärzte existiert.

Zudem können ähnliche Analysen auch in Zukunft wichtige Informationen über klinikinterne Abläufe liefern und damit Standards verbessern. Um jedoch in kürzerer Zeit geeignete Daten schneller aufbereiten zu können, sollten noch mehr Daten als bisher in elektronischer Form vorliegen. Der IT-Einsatz im Krankenhaus trägt zu effektiveren Organisations- und Entscheidungsprozessen bei (Haas und Kuhn, 2011). Die Verwendung konventioneller Papierakten führt immer wieder zu Informationslücken in der medizinischen Versorgung. Elektronische Akten können dabei redundante Datenbestände reduzieren und dazu führen, dass der Patient mehr in den Mittelpunkt rückt (Hübner, 2004). 


\section{Zusammenfassung}

In den letzten Jahren ließ sich der Trend beobachten, dass immer mehr Patienten den Notdienst, d. h. die medizinische Versorgung außerhalb der üblichen Sprechzeiten, in Anspruch nehmen. Die Versorgung der gravierenden Notfälle im Hals-Nasen-Ohren-Bereich übernimmt in Göttingen nach Bestimmung der Kassenärztlichen Vereinigung Niedersachsen die HNO-Klinik der Universitätsmedizin Göttingen. Ziel dieser Untersuchung war es, das Notfallaufkommen der HNOKlinik zu analysieren und zu überprüfen, welche Rolle der HNO-Notdienst tatsächlich innerhalb der medizinischen Versorgung außerhalb der Sprechzeiten einnimmt.

Dafür wurden retrospektiv 2.396 Patientenfälle aus dem Jahr 2011 untersucht, die außerhalb der Sprechzeiten im Notdienst der Universitätsmedizin Göttingen behandelt wurden. Neben den Patientenzahlen wurden Aufnahmeart, Diagnosen, Altersstruktur, Konsile, Einzugsgebiet, Stoßzeiten, Operationen, Nachsorge, Risikofaktoren und Komplikationen ausgewertet.

Der Schwerpunkt im HNO-Notdienst der Universitätsmedizin Göttingen lag auf der ambulanten Notfallversorgung. Das Verhältnis von ambulanter zu stationärer Versorgung betrug sieben zu eins. Es lag ein breites Spektrum an Diagnosen vor. Die häufigsten drei Diagnosen bei den ambulanten Patienten waren „Epistaxis“ (11,4\%), „Eitrige Otitis media“ (8,7 \%) und „Otitis externa (8,3 \%), bei den stationären Patienten hingegen „Epistaxis“ (10,1 \%), „Peritonsillarabszess" (7,4 \%) und „Nachblutungen“ (6,0 \%). Insgesamt handelte es sich bei Notdienst-Patienten um eine junge Patientengruppe, wobei das durchschnittliche Alter der ambulanten Patienten mit 39,4 Jahren unter dem der stationären Patienten mit einem Durchschnittsalter von 43,4 Jahren lag. Während bei den 
ambulanten Patienten die Altersgruppe der 21- bis 25- Jährigen am stärksten vertreten war, war es die Altersgruppe der 71- bis 75- Jährigen bei den stationären Patienten.

Der größte Teil der ambulanten Patienten (38\%) hatte seinen Erstwohnsitz im Stadtgebiet Göttingen, während die stationären Patienten vermehrt aus dem direkten Umland kamen (31,8 \%). Es konnten saisonale Schwankungen hinsichtlich der ambulanten Notdienstbesuche festgestellt werden. Im Monat Mai kamen die meisten Patienten, die wenigsten im September. Sowohl die ambulanten, als auch die stationären Patienten suchten den Notdienst überwiegend am Samstag und Sonntag auf (59,4 \% bzw. 48 \%). Insbesondere die Einnahme von Antikoagulanzien, das Vorhandensein von Allgemeinerkrankungen und ein erhöhtes Lebensalter ließen sich als Risikofaktoren für einen Teil der Notdienstpatienten feststellen. Nachblutungen stellten eine häufige Komplikation dar, mit der der HNO-Notdienst konfrontiert war. Sie ging bei 21 von 22 Fällen auf eine vorangegangene Tonsillektomie zurück.

Der HNO-Notdienst übernimmt eine wichtige Rolle in der Versorgung außerhalb der Sprechzeiten. Nicht nur schwere Erkrankungen, sondern auch ein hoher Anteil an leichten Erkrankungen wird therapiert. Vor diesem Hintergrund sollte die Aufgabe der HNO-Klinik geklärt werden, da es nur so möglich ist, die Patientenströme gezielt zu den richtigen Stellen zu lenken. 


\section{Anhang}

\begin{tabular}{|c|c|c|c|}
\hline $\begin{array}{l}\text { ICD-10- } \\
\text { Schlüssel }\end{array}$ & $\begin{array}{l}\text { Verwendeter Dia- } \\
\text { gnosenbegriff }\end{array}$ & $\begin{array}{l}\text { Diagnosenobergruppe } \\
\text { nach ICD-10 }\end{array}$ & $\begin{array}{l}\text { Diagnosenuntergruppe } \\
\text { nach ICD-10 }\end{array}$ \\
\hline B27 & Mononukleose & $\begin{array}{l}\text { Infektiöse Mononukleo- } \\
\text { se }\end{array}$ & $\begin{array}{l}\text { - Mononukleose durch } \\
\text { Gammaherpesviren } \\
\text { - Mononukleose durch } \\
\text { Zytomegaleviren } \\
\text { - Sonstige infektiöse } \\
\text { Mononukleose } \\
\text { - Infektiöse Mononu- } \\
\text { kleose, nicht näher be- } \\
\text { zeichnet }\end{array}$ \\
\hline $\mathrm{H} 60$ & Otitis externa & Otitis externa & $\begin{array}{l}\text { - Abszess des äußeren } \\
\text { Ohres } \\
\text { - Phlegmone des } \\
\text { äußeren Ohres } \\
\text { - Sonstige infektiöse } \\
\text { Otitis externa } \\
\text { - Akute Otitis externa, } \\
\text { nichtinfektiös } \\
\text { - Sonstige Otitis exter- } \\
\text { na (chronische Otitis } \\
\text { externa) } \\
\text { - Otitis externa, nicht } \\
\text { näher bezeichnet }\end{array}$ \\
\hline $\mathrm{H} 61$ & Zeruminalpfropf & $\begin{array}{l}\text { Sonstige Krankheiten } \\
\text { des äußeren Ohres }\end{array}$ & $\begin{array}{l}\text { - Perichondritis des } \\
\text { äußeren Ohres }\end{array}$ \\
\hline
\end{tabular}

Fortsetzung folgt auf der nächsten Seite 


\begin{tabular}{|c|c|c|c|}
\hline $\begin{array}{l}\text { ICD-10- } \\
\text { Schlüssel }\end{array}$ & $\begin{array}{l}\text { Verwendeter Dia- } \\
\text { gnosenbegriff }\end{array}$ & $\begin{array}{l}\text { Diagnosenobergruppe } \\
\text { nach ICD-10 }\end{array}$ & $\begin{array}{l}\text { Diagnosenuntergruppe } \\
\text { nach ICD-10 }\end{array}$ \\
\hline & & & $\begin{array}{l}\text { - Nichtinfektiöse Krank- } \\
\text { heiten der Ohrmuschel } \\
\text { - Zeruminalpfropf } \\
\text { - Sonstige nicht näher } \\
\text { bezeichnete Krankheiten } \\
\text { des äußeres Ohres } \\
\text { - Krankheit des äußeren } \\
\text { Ohres, nicht näher be- } \\
\text { zeichnet }\end{array}$ \\
\hline H65 & $\begin{array}{l}\text { Nichteitrige Otitis } \\
\text { media }\end{array}$ & $\begin{array}{l}\text { Nichteitrige Otitis me- } \\
\text { dia }\end{array}$ & $\begin{array}{l}\text { - Akute seröse Otitis } \\
\text { media } \\
\text { - Akute nichteitrige } \\
\text { Otitis media } \\
\text { - Sonstige akute nich- } \\
\text { teitrige Otitis media } \\
\text { - Chronische seröse } \\
\text { Otitis media } \\
\text { - Sonstige chronische } \\
\text { nichteitrige Otitis media } \\
\text { - Nichteitrige Otitis } \\
\text { media, nicht näher be- } \\
\text { zeichnet }\end{array}$ \\
\hline H66 & $\begin{array}{l}\text { Eitrige Otitis me- } \\
\text { dia }\end{array}$ & $\begin{array}{l}\text { Eitrige und nicht näher } \\
\text { bezeichnete Otitis me- } \\
\text { dia }\end{array}$ & $\begin{array}{l}\text {-Akute eitrige Otitis } \\
\text { media } \\
\text { - Chronische mesotym- } \\
\text { panale eitrige Otitis } \\
\text { media } \\
\text { - Chronische epitym- } \\
\text { panale Otitis media } \\
\text { - Sonstige chronische } \\
\text { eitrige Otitis media }\end{array}$ \\
\hline
\end{tabular}

Fortsetzung folgt auf der nächsten Seite 


\begin{tabular}{|c|c|c|c|}
\hline $\begin{array}{l}\text { ICD-10- } \\
\text { Schlüssel }\end{array}$ & $\begin{array}{l}\text { Verwendeter Dia- } \\
\text { gnosenbegriff }\end{array}$ & $\begin{array}{l}\text { Diagnosenobergruppe } \\
\text { nach ICD-10 }\end{array}$ & $\begin{array}{l}\text { Diagnosenuntergruppe } \\
\text { nach ICD-10 }\end{array}$ \\
\hline & & & $\begin{array}{l}\text { • Eitrige Otitis media, } \\
\text { nicht näher bezeichnet } \\
\text { • Otitis media, nicht } \\
\text { näher bezeichnet }\end{array}$ \\
\hline H69 & $\begin{array}{l}\text { Krankheiten Tuba } \\
\text { auditiva }\end{array}$ & $\begin{array}{l}\text { Sonstige Krankheiten } \\
\text { der Tuba auditiva }\end{array}$ & $\begin{array}{l}\text { Sonstige nicht näher } \\
\text { bezeichnete Krankheiten } \\
\text { der Tuba auditiva }\end{array}$ \\
\hline H81 & $\begin{array}{l}\text { Störungen Vestibu- } \\
\text { larfunktion }\end{array}$ & $\begin{array}{l}\text { Störungen der Vestibu- } \\
\text { larfunktion }\end{array}$ & $\begin{array}{l}\text { - Meniere-Krankheit } \\
\text { - Benigner paroxysma- } \\
\text { ler Schwindel } \\
\text { - Neuropathia vestibu- } \\
\text { laris } \\
\text { - Sonstiger peripherer } \\
\text { Schwindel } \\
\text { - Schwindel zentralen } \\
\text { Ursprungs } \\
\text { - Störungen der Ves- } \\
\text { tibularfunktion, nicht } \\
\text { näher bezeichnet }\end{array}$ \\
\hline H91 & Hörverlust/Hörsturz & Sonstiger Hörverlust & $\begin{array}{l}\text { • Ideopathischer } \\
\text { Hörsturz } \\
\text { • Hörverlust, nicht } \\
\text { näher bezeichnet }\end{array}$ \\
\hline H93 & Tinnitus aurium & $\begin{array}{l}\text { Sonstige Krankheiten } \\
\text { des Ohres, andernorts } \\
\text { nicht klassifiziert }\end{array}$ & $\begin{array}{l}\text { - Tinnitus aurium } \\
\text { • Sonstige abnorme } \\
\text { Hörempfindungen } \\
\text { - Krankheit des Ohres, } \\
\text { nicht näher bezeichnet }\end{array}$ \\
\hline J01 & Akute Sinusitis & Akute Sinusitis & $\begin{array}{l}\text { - Akute Sinusitis maxil- } \\
\text { laris }\end{array}$ \\
\hline
\end{tabular}

Fortsetzung folgt auf der nächsten Seite 


\begin{tabular}{|c|c|c|c|}
\hline $\begin{array}{l}\text { ICD-10- } \\
\text { Schlüssel }\end{array}$ & $\begin{array}{l}\text { Verwendeter Dia- } \\
\text { gnosenbegriff }\end{array}$ & $\begin{array}{l}\text { Diagnosenobergruppe } \\
\text { nach ICD-10 }\end{array}$ & $\begin{array}{l}\text { Diagnosenuntergruppe } \\
\text { nach ICD-10 }\end{array}$ \\
\hline & & & $\begin{array}{l}\text { - Akute Sinusitis fronta- } \\
\text { lis } \\
\text { - Akute Sinusitis etho- } \\
\text { moidalis } \\
\text { - Akute Pansinusitis } \\
\text { - Sonstige akute Sinusi- } \\
\text { tis } \\
\text { - Akute Sinusitis, nicht } \\
\text { näher bezeichnet }\end{array}$ \\
\hline J02 & Akute Pharyngitis & Akute Pharyngitis & $\begin{array}{l}\text { - Akute Pharyngitis } \\
\text { durch sonstige nicht } \\
\text { näher bezeichnete Erre- } \\
\text { ger } \\
\text { - Akute Pharyngitis, } \\
\text { nicht näher bezeichnet }\end{array}$ \\
\hline J03 & Akute Tonsillitis & Akute Tonsillitis & $\begin{array}{l}\text { - Akute Tonsillitis, } \\
\text { nicht näher bezeichnet }\end{array}$ \\
\hline J04 & $\begin{array}{l}\text { Akute Laryngi- } \\
\text { tis/Tracheitis }\end{array}$ & $\begin{array}{l}\text { Akute Laryngitis und } \\
\text { Tracheitis }\end{array}$ & $\begin{array}{l}\text { - Akute Laryngitis } \\
\text { - Akute Tracheitis } \\
\text { - Akute Laryngotrachei- } \\
\text { tis }\end{array}$ \\
\hline J06 & Atemwegsinfektion & $\begin{array}{l}\text { Akute Infektionen an } \\
\text { mehreren oder nicht } \\
\text { näher bezeichneten Lo- } \\
\text { kalisationen der oberen } \\
\text { Atemwege }\end{array}$ & $\begin{array}{l}\text { - Akute Laryngopha- } \\
\text { ryngitis } \\
\text { - Sonstige akute Infek- } \\
\text { tionen an mehreren Lo- } \\
\text { kalisationen der oberen } \\
\text { Atemwege }\end{array}$ \\
\hline
\end{tabular}

Fortsetzung folgt auf der nächsten Seite 


\begin{tabular}{|c|c|c|c|}
\hline $\begin{array}{l}\text { ICD-10- } \\
\text { Schlüssel }\end{array}$ & $\begin{array}{l}\text { Verwendeter Dia- } \\
\text { gnosenbegriff }\end{array}$ & $\begin{array}{l}\text { Diagnosenobergruppe } \\
\text { nach ICD-10 }\end{array}$ & $\begin{array}{l}\text { Diagnosenuntergruppe } \\
\text { nach ICD-10 }\end{array}$ \\
\hline & & & $\begin{array}{l}\text { - Akute Infektionen der } \\
\text { oberen Atemwege, nicht } \\
\text { näher bezeichnet }\end{array}$ \\
\hline J32 & chronische Sinusitis & Chronische Sinusitis & $\begin{array}{l}\text { - Chronische Sinusitis } \\
\text { maxillaris } \\
\text { - Chronische Sinusitis } \\
\text { frontalis } \\
\text { - Chronische Sinusitis } \\
\text { ethmoidalis } \\
\text { - Chronische Sinusitis } \\
\text { sphenoidalis } \\
\text { - Chronische Pansinusi- } \\
\text { tis } \\
\text { - Sonstige chronische } \\
\text { Sinusitis } \\
\text { - Chronische Sinusitis, } \\
\text { nicht näher bezeichnet }\end{array}$ \\
\hline J34 & $\begin{array}{l}\text { Krankheiten Na- } \\
\text { se/NNH }\end{array}$ & $\begin{array}{l}\text { Sonstige Krankheiten } \\
\text { der Nase und Nasenne- } \\
\text { benhöhlen }\end{array}$ & $\begin{array}{l}\text { - Abszess, Furunkel und } \\
\text { Karbunkel der Nase } \\
\text { - Nasenseptumdeviation } \\
\text { - Hypertrophie der Na- } \\
\text { senmuscheln } \\
\text { - Sonstige nicht näher } \\
\text { bezeichnete Krankheiten } \\
\text { der Nase und Nasenne- } \\
\text { benhöhlen }\end{array}$ \\
\hline J35 & $\begin{array}{l}\text { chronische Krank- } \\
\text { heiten Tonsillen }\end{array}$ & $\begin{array}{l}\text { Chronische Krankhei- } \\
\text { ten der Gaumenman- } \\
\text { deln und Rachenman- } \\
\text { deln }\end{array}$ & $\begin{array}{l}\text { - Chronische Tonsillitis } \\
\text { - Hyperplasie der Gau- } \\
\text { menmandeln }\end{array}$ \\
\hline
\end{tabular}

Fortsetzung folgt auf der nächsten Seite 


\begin{tabular}{|c|c|c|c|}
\hline $\begin{array}{l}\text { ICD-10- } \\
\text { Schlüssel }\end{array}$ & $\begin{array}{l}\text { Verwendeter Dia- } \\
\text { gnosenbegriff }\end{array}$ & $\begin{array}{l}\text { Diagnosenobergruppe } \\
\text { nach ICD-10 }\end{array}$ & $\begin{array}{l}\text { Diagnosenuntergruppe } \\
\text { nach ICD-10 }\end{array}$ \\
\hline & & & $\begin{array}{l}\text { - Hyperplasie der Ra- } \\
\text { chenmandeln } \\
\text { - Hyperplasie der Gau- } \\
\text { menmandeln mit Hyper- } \\
\text { plasie der Rachenman- } \\
\text { del } \\
\text { - Sonstige chronische } \\
\text { Krankheiten der Gau- } \\
\text { menmandeln und der } \\
\text { Rachenmandel }\end{array}$ \\
\hline J36 & Peritonsillarabszess & Peritonsillarabszess & \\
\hline $\mathrm{J} 38$ & Larynxödem & $\begin{array}{l}\text { Krankheiten der } \\
\text { Stimmlippen und des } \\
\text { Kehlkopfes, anderenorts } \\
\text { nicht klassifiziert }\end{array}$ & - Larynxödem \\
\hline K11 & $\begin{array}{l}\text { Speicheldrüsen- } \\
\text { erkrankung }\end{array}$ & $\begin{array}{l}\text { Krankheiten der Spei- } \\
\text { cheldrüsen }\end{array}$ & $\begin{array}{l}\text { - Sialadenitis } \\
\text { - Speicheldrüsenfistel } \\
\text { - Sialolithiasis }\end{array}$ \\
\hline R04 & Epistaxis & $\begin{array}{l}\text { Blutung aus den Atem- } \\
\text { wegen }\end{array}$ & $\begin{array}{l}\text { - Epistaxis } \\
\text { • Blutung aus dem Ra- } \\
\text { chen } \\
\text { - Blutung aus sonstigen } \\
\text { Lokalisationen in den } \\
\text { Atemwegen }\end{array}$ \\
\hline $\mathrm{R} 42$ & $\begin{array}{l}\text { Schwindel und } \\
\text { Taumel }\end{array}$ & Schwindel und Taumel & \\
\hline $\mathrm{S} 02$ & $\begin{array}{l}\text { Fraktur des } \\
\text { Schädels }\end{array}$ & $\begin{array}{l}\text { Fraktur des Schädels } \\
\text { und der Ge- } \\
\text { sichtsschädelknochen }\end{array}$ & $\begin{array}{l}\text { - Schädeldachfraktur } \\
\text { • Schädelbasisfraktur } \\
\text { - Nasenbeinfraktur }\end{array}$ \\
\hline
\end{tabular}

Fortsetzung folgt auf der nächsten Seite 


\begin{tabular}{|c|c|c|c|}
\hline $\begin{array}{l}\text { ICD-10- } \\
\text { Schlüssel }\end{array}$ & $\begin{array}{l}\text { Verwendeter Dia- } \\
\text { gnosenbegriff }\end{array}$ & $\begin{array}{l}\text { Diagnosenobergruppe } \\
\text { nach ICD-10 }\end{array}$ & $\begin{array}{l}\text { Diagnosenuntergruppe } \\
\text { nach ICD-10 }\end{array}$ \\
\hline & & & $\begin{array}{l}\text { - Fraktur des Orbitabo- } \\
\text { dens } \\
\text { - Fraktur des Jochbeins } \\
\text { und des Oberkiefers }\end{array}$ \\
\hline S09 & Verletzung Kopf & $\begin{array}{l}\text { Sonstige und nicht } \\
\text { näher bezeichnete Ver- } \\
\text { letzungen des Kopfes }\end{array}$ & $\begin{array}{l}\text { - Verletzung von Mus- } \\
\text { keln und Sehnen des } \\
\text { Kopfes } \\
\text { - Traumatische Trom- } \\
\text { melfellruptur } \\
\text { - Nicht näher bezeich- } \\
\text { nete Verletzung des } \\
\text { Kopfes }\end{array}$ \\
\hline $\mathrm{T} 16$ & Fremdkörper Ohr & Fremdkörper im Ohr & \\
\hline $\mathrm{T} 17$ & $\begin{array}{l}\text { Fremdkörper } \\
\text { Atemwege }\end{array}$ & $\begin{array}{l}\text { Fremdkörper in den } \\
\text { Atemwegen }\end{array}$ & $\begin{array}{l}\text { - Fremdkörper in einer } \\
\text { Nasennebenhöhle } \\
\text { • Fremdkörper im Na- } \\
\text { senloch } \\
\text { • Fremdkörper im Na- } \\
\text { senloch } \\
\text { • Fremdkörper im Ra- } \\
\text { chen } \\
\text { - Fremdkörper im Kehl- } \\
\text { kopf } \\
\text { - Fremdkörper in der } \\
\text { Trachea } \\
\text { • Fremdkörper in den } \\
\text { Atemwegen, Teil nicht } \\
\text { näher bezeichnet }\end{array}$ \\
\hline $\mathrm{T} 18$ & $\begin{array}{l}\text { Fremdkörper Ver- } \\
\text { dauungstrakt }\end{array}$ & $\begin{array}{l}\text { Fremdkörper im Ver- } \\
\text { dauungstrakt }\end{array}$ & $\begin{array}{l}\text { - Fremdkörper im } \\
\text { Mund } \\
\text { • Fremdkörper im } \\
\text { Ösophagus }\end{array}$ \\
\hline
\end{tabular}

Fortsetzung folgt auf der nächsten Seite 


\begin{tabular}{|c|c|c|c|}
\hline $\begin{array}{l}\text { ICD-10- } \\
\text { Schlüssel }\end{array}$ & $\begin{array}{l}\text { Verwendeter Dia- } \\
\text { gnosenbegriff }\end{array}$ & $\begin{array}{l}\text { Diagnosenobergruppe } \\
\text { nach ICD-10 }\end{array}$ & $\begin{array}{l}\text { Diagnosenuntergruppe } \\
\text { nach ICD-10 }\end{array}$ \\
\hline & & & $\begin{array}{l}\text { - Fremdkörper an sons- } \\
\text { tigen und mehreren } \\
\text { Lokalisationen des Ver- } \\
\text { dauungstraktes } \\
\text { - Fremdkörper im Ver- } \\
\text { dauungstrakt, Teil nicht } \\
\text { näher bezeichnet }\end{array}$ \\
\hline $\mathrm{T} 81$ & Nachblutungen & $\begin{array}{l}\text { Komplikation bei Ein- } \\
\text { griffen, andernorts } \\
\text { nicht klassifiziert }\end{array}$ & $\begin{array}{l}\text { - Blutung und } \\
\text { Hämatom als Kompli- } \\
\text { kation eines Eingriffes, } \\
\text { andernorts nicht klassifi- } \\
\text { ziert } \\
\text { - Schock während oder } \\
\text { als Folge eines Eingrif- } \\
\text { fes, anderenorts nicht } \\
\text { klassifiziert }\end{array}$ \\
\hline
\end{tabular}

Tabellenende 


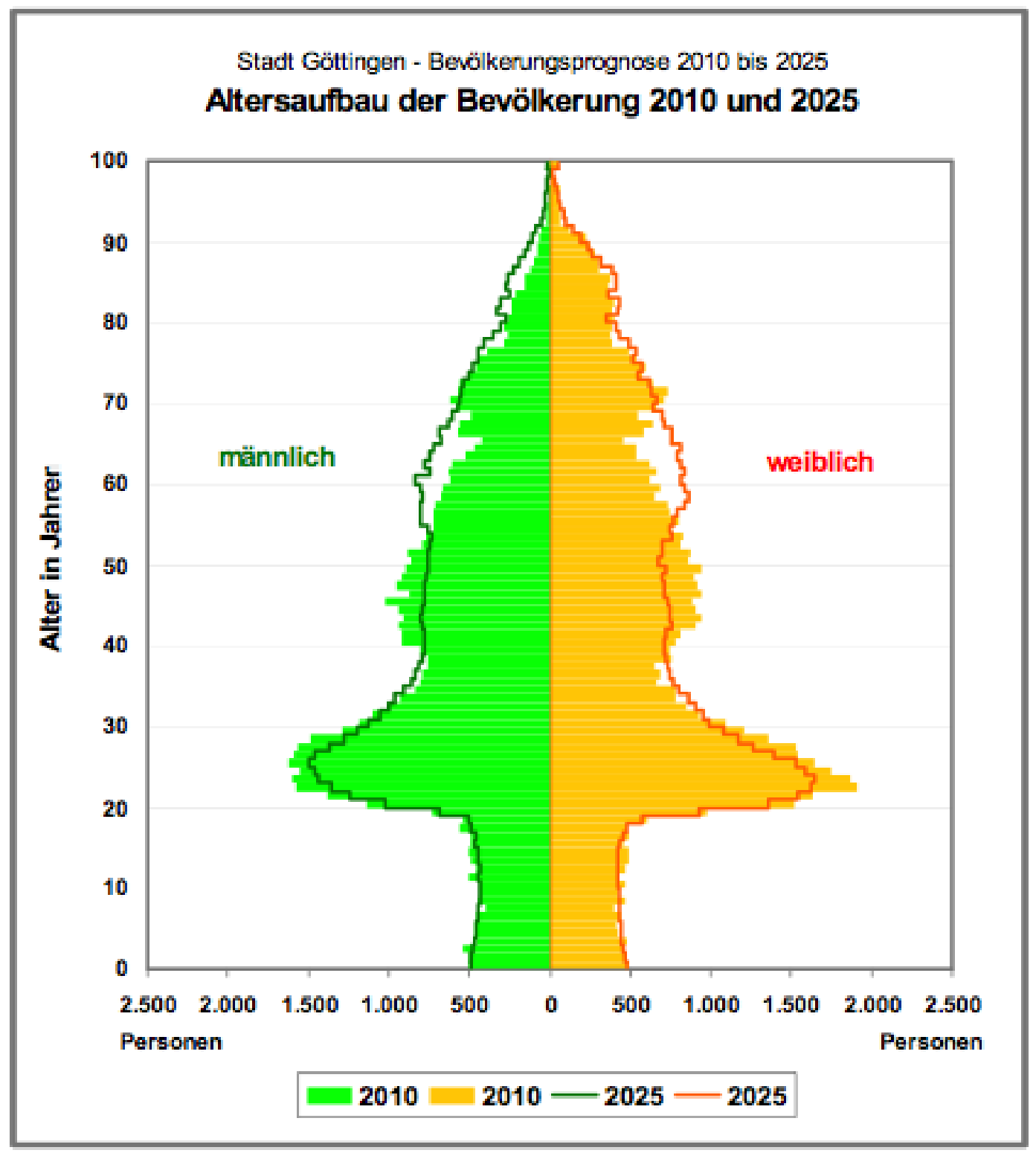

Abbildung 6.1: Altersaufbau der Bevölkerung der Stadt Göttingen und Bevölkerungsprognose bis 2025. (c) Fachdienst Statistik und Wahlen, Stadt Göttingen. Mit freundlicher Genehmigung der Stadt Göttingen. Quelle: http://www.goesis.goettingen.de/pdf/Aktuell33.pdf 


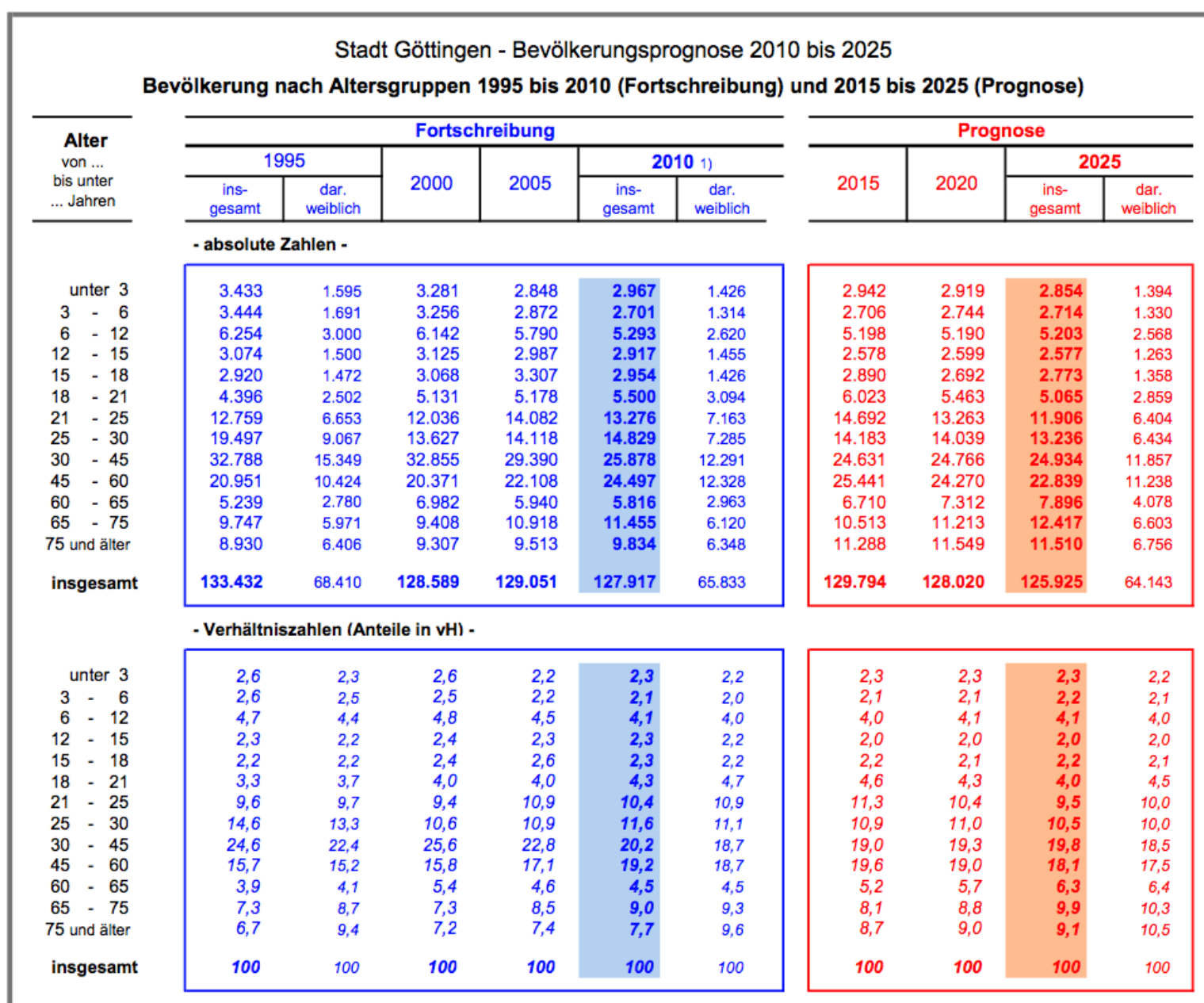

Abbildung 6.2: Bevölkerung nach Altersgruppen 1995 bis 2010 (Fortschreibung) und 2015 bis 2025 (Prognose). (C) Fachdienst für Statistik und Wahlen. Mit freundlicher Genehmigung der Stadt Göttingen. Quelle: http://www.goesis.goettingen.de/pdf/Aktuell33.pdf 
Tabelle 6.1: Median, Mittelwert und Standardabweichung des Lebensalters in Jahren für die ersten zehn Diagnosegruppen der ambulanten Patienten (in Jahren)

\begin{tabular}{|c||c|c|c|}
\hline Gruppe & Median & Mittelwert & Standardabweichung \\
\hline \hline „Epistaxis“ & 63 & 57 & 25 \\
\hline „Eitrige Otitis media“" & 28 & 28 & 17 \\
\hline „Otitis externa“ & 31 & 34 & 17 \\
\hline „Zeruminalpfropf“ & 44 & 49 & 19 \\
\hline "Hörverlust/Hörsturz" & 47 & 48 & 19 \\
\hline "Akute Pharyngitis" & 30 & 33 & 14 \\
\hline „Tinnitus aurium“ & 42 & 43 & 25 \\
\hline „Fremdkörper Atemwege“ & 20 & 26 & 10 \\
\hline "Akute Tonsilitis“ & 26 & 27 & 11 \\
\hline "Chronische Sinusitis“ & 35 & 35 & \\
\hline
\end{tabular}

Tabelle 6.2: Median, Mittelwert und Standardabweichung des Lebensalters in Jahren für die häufigsten fünf Diagnosegruppen der stationären Patienten (in Jahren)

\begin{tabular}{|c||c|c|c|}
\hline Gruppe & Median & Mittelwert & Standardabweichung \\
\hline \hline „Epistaxis“ & 70 & 61 & 23 \\
\hline "Peritonsillarabszess“ & 31 & 37 & 14 \\
\hline "Nachblutungen“ & 22 & 24 & 15 \\
\hline „Störungen der Vestibularfunktion“" & 50 & 51 & 15 \\
\hline "chronische Krankheiten der Tonsillen“ & 4 & 9 & 9 \\
\hline
\end{tabular}




\section{Abbildungsverzeichnis}

$3.1 \quad$ Registrierte Patientenzahlen der Jahre 2010, 2011, 2012 auf Station 6012. . . 17

$3.2 \quad$ Prozentuales Verhältnis der ambulanten zu den stationären Patienten . . . . 18

$3.3 \quad$ Häufigkeiten der ambulanten und stationären Patienten in Altersklassen . . . 22

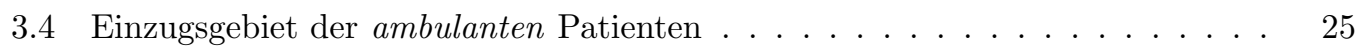

$3.5 \quad$ Einzugsgebiet der stationären Patienten . . . . . . . . . . . . . . 26

$3.6 \quad$ Häufigkeit der Patientenkontakte im Jahresverlauf $\ldots \ldots \ldots$

$3.7 \quad$ Häufigkeit der ambulanten Patientenkontakte innerhalb der Woche . . . . . . 29

3.8 Häufigkeiten der stationären Patientenkontakte innerhalb der Woche . . . . . 29

3.9 Boxplots für das Alter der ambulanten Patienten . . . . . . . . . . . . . . . 31

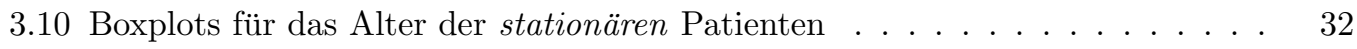

3.11 Häufigkeiten von Risikofaktoren bei Epistaxis-Patienten . . . . . . . . . . . . 34

3.12 Operative Eingriffe nach Häufigkeiten bei den stationären Patienten . . . . . 36

3.13 Verteilung von „Nachblutungen“ auf die jeweiligen postoperativen Tage . . . 37

3.14 Wiederkehrer innerhalb der zwei Monate nach Notdienst-Besuch . . . . . . . 41

3.15 Wiederkehrer: Häufigkeiten der operativen Eingriffe. . . . . . . . . . . . . . . 42

$6.1 \quad$ Altersaufbau der Bevölkerung der Stadt Göttingen und Bevölkerungsprognose

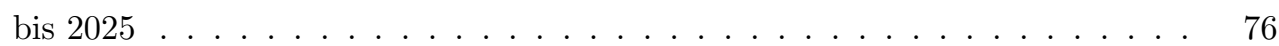

6.2 Bevölkerung nach Altersgruppen für die Jahre 1995 bis 2010 (Fortschreibung) und 2015 (Prognose) $\ldots \ldots \ldots \ldots$. . . . . . . . . . . . . . 77 


\section{Tabellenverzeichnis}

3.1 Die zehn häufigsten Diagnosen der ambulanten Patienten . . . . . . . . . . 20

$3.2 \quad$ Die fünf häufigsten Diagnosen der stationären Patienten . . . . . . . . . . . 20

$3.3 \quad$ Einwohnerzahlen nach Postleitzahlengruppen . . . . . . . . . . . . . . . . 23

3.4 Häufigkeiten der Einnahme von Antikoagulanzien . . . . . . . . . . . . . . . . 35

3.5 Relative und absolute Häufigkeiten von Konsilen der stationären Patienten . 39

6.1 Median, Mittelwert und Standardabweichung des Lebensalters für die Patienten der zehn häufigsten ambulanten Diagnosen . . . . . . . . . . . . . 78

$6.2 \quad$ Median, Mittelwert und Standardabweichung des Lebensalters für die Patienten der fünf häufigsten stationären Diagnosen . . . . . . . . . . . . . . . . 78 


\section{Literaturverzeichnis}

Ärztekammer Niedersachsen (2007): Bereitschaftsdienstordnung der Kassenärztlichen Vereinigung Niedersachsen. https://www. aekn.de.

Bermüller C, Bender M, Brögger C, Retereit F, Schulz M (2014): Epistaxis-bei Antikoagulation-eine klinische und ökonomische Herausforderung. Laryngorhinootologie $\underline{93}, 249-255$.

Bertram E, Farthmann E (1981): Notwendigkeit und Anwendungsgebiet retrospektiver Analysen in einer Zeit der prospektiven kontrollierten Studien. Langenbecks Arch Chir 355, 3937 .

Bitzer E, Grobe T, Dörning H, Schwartz F: Kindergesundheit stärken. Springer Verlag, Berlin 2009.

Blum K, Offermanns M (2009): Krankenhäuser zwischen Innovations- und Kostendruck. Das Krankenhaus $\underline{4}, 295-302$.

Bolz M: Zum Stellenwert der Notfallambulanz der HNO-Klinik des Universitätsklinikums Köln in der Versorgung ambulanter Patienten. Med. Diss. Köln 2009.

Busse R, Blümel M, Ognyanova D: Das deutsche Gesundheitssystem: Akteure, Daten, Analysen. 2. Auflage; MWV Medizinisch Wissenschaftliche Verlagsgesellschaft, Berlin 2013.

Dagan E, Wolf M, Migirov L (2012): Why do geriatric patients attend otolaryngology emergency rooms? Isr Med Assoc J 14, 633-6.

Feldmann H: HNO-Notfälle. 2. Auflage; Springer Verlag, Berlin 1973.

Fischer W: Notfallvergütung im Krankenhaus. 1. Auflage; Zentrum für Informatik und wirtschaftliche Medizin, Wolfertswil, 2009.

Folz B, Kanne M, Werner J (2008): Aktuelle Aspekte zur Epistaxis. HNO 2008 56, 1157-1166.

Gallo A, Moi R, Minni A, Simonelli M, de Vincentis M (2000): Otorhinolaryngology emergency unit care: the experience of a large university hospital in Italy. Ear Nose and Throat J $\underline{79}, 155-158,160$. 
Haas P, Kuhn K: Krankenhausinformationssysteme-Ziele, Nutzen, Auswahl. 4. Auflage; Springer Verlag, Berlin 2011.

Hagl S: Schnelleinstieg Statistik. 1. Auflage, Rudolf Haufe Verlag, München 2008.

Hijano R, Hernandez A, Martinez-Arias A, Homs I, Navarrete M (2009): Epidemiological study of emergency services at a tertiary care center. Acta Otorrinolaringol Esp $\underline{60}, 32-7$.

Hoppe J, Koehler A, Pfeiffer D, Brenner G, Klar R: Internationale statistische Klassifikation der Krankheiten und verwandter Gesundheitsprobleme. 10. Auflage; Deutscher Ärzteverlag, Köln 2008.

Hübner U (2004): 80 Prozent der Krankenhäuser immer noch ohne elektronischen Patientenakte. Krankenhaus IT Journal o Jg 75.

Kanne M: Pathogenese und Therapie der Epistaxis - Eine prospektive Analyse von 108 konsekutiven Fällen an der HNO-Klinik der Philipps-Universität Marburg. Med. Diss. Marburg 2007.

Kassenärztliche Vereinigung Schleswig-Holstein (2009): Ärztlicher Bereitschaftsdienst. http: //www.kvsh.de/index . php?StoryID=64.

Kelly LJ, Birtwhistle R (1993): Is this problem urgent? Can Fam Physician $\underline{39}, 1345-52$.

Killinger E: Die Besonderheiten der Arzthaftung im medizinischen Notfall. 1. Auflage; Springer Verlag, Heidelberg 2009.

Kitcher E, Jangu A, Baidoo K (2007): Emergency ear, nose and throat admissions at the Korle-Bu Teaching Hospital. Ghana medical journal 41, 9-11.

Knuth P (1998): Notarzt und Notfallarzt. Internist $\underline{39}$, 120-123.

Kotecha B (1996): Management of epistaxis: a national survey. Ann R Coll Surg Engl $\underline{78}, 444-$ 446.

Lasisi O, Imam Z, Adesun A (2007): Otorhinolaryngologic Emergencies in Nigeria, Subsaharan Africa: Implication for training. Association of Surgeons of East Africa and College of Surgeons of East Central and Southern Africa 16, 592-595.

Lorenz W (1981): Retrospektive Studien in der Chirurgie. Langenbecks Arch Chir $\underline{355}$, 387391.

Luthe EW (2013): Kommunale Verantwortung für das Rettungswesen. Kommunale Gesundheitslandschaften. Springer. 
Morrmann F (2007): Die Bedeutung der Kultur eines Krankenhauses. Diplomarbeit, Köln 2007.

Muehlbauer B, Kellerhoff F, Zukunftsperspektiven der Gesundheitswirtschaft. Lit Verlag, Berlin 2012.

Nagel P, Gürkov R: Hals-Nasen-Ohren-Heilkunde. 2. Auflage; Elsevier, München, 2009.

Perez O, Fernandez L, J MG, J SN, A MB (1995): An outpatient study of ENT (Otorhinolaryngology) emergencies at a general hospital. Acta Otorrinolaringol $\underline{46}$, 298-304.

Probst R, Grevers G, Iro H: Hals-Nasen-Ohrenheilkunde. 3. Auflage; Georg Thieme Verlag, Stuttgart, 2008.

Pschyrembel W: Klinisches Woerterbuch. 259. Auflage; de Gruyter, Berlin 2002.

Rivero V, Ruiz G, Palomino A, Romero G, Hernandez C, Garcia M, Yanez T, Huelva A (2005): Considerations about ENT emergencies. Analysis of 30000 patients assisted in 10 years. Acta Otorrinolaringol Esp $\underline{56}, 198-201$.

Robra BP, Huke T, Swart E (2013): Die Abbildung von Notfall-Patienten in Routinedaten. Technischer Bericht, Otto von Guericke Universtiät Magdeburg.

Rosenmayr L: Hoffnung Alter. 2. Auflage; Facultas Verlags- und Buchhandels AG, Wien 2006.

Saha S, Chandra S, Mondal PK, Das S, Mishra S, Rashid MA, Mondal A (2005): Emergency Otorhinolarygolocal Cases in Medical College, Kolkata - A Statistical Analysis. Indian J Otolaryngol Head Neck Surg $\underline{57}, 219-225$.

Scheckenbach K, Bier H, Hoffmann TK, Windfuht JP, Bas M, Laws HJ, Plettenberg C, Wagenmann M (2008): Risiko von Blutungen nach Adenotomie und Tonsillektomie. HNO $\underline{56}, 312-320$.

Schlicht L, Guntinas-Lichius O (2012): Stellenwert der Notfallambulanz der HNO-Klinik Jena in der ambulanten Patientenversorgung. HNO $2012 \underline{60}, 1-4$.

Schmergal C (2014): Blaue Tage. Der Spiegel 33, 64-65.

Schmidt H, Schmiz A, Stasche N, Hörmann K (1996): Operativ versorgte Nachblutungen nach Tonsillektomie. Laryng Rhinol Otol $\underline{75}, 447-454$.

Simmen D, Heinz B (1998): Epistaxis-Strategie - Erfahrungen der letzten 360 Hospitalisationen. Laryngo Rhino Otol 구, 100-106. 
Staffa G: Analyse der Inanspruchnahme des kassenärztlichen Notfalldienstes in einem Hamburger Notfalldienstbezirk im Zeitraum August 1996 bis August 1997. Med. Diss. Hamburg 2007.

Steland A, Basiswissen Statistik. Springer, Berlin 2007, 2. Auflage.

Symvoulakis E, Klinis S, Alegakis A, Kyrmizakis D, Drivas E, Rachiotis G, Philalithis A, Velegrakis G (2006): Epidemiologic profile of otorhinolaryngological, head and neck disorders in a tertiary hospital unit in Greece: a challenge for general practitioners? BMC Ear Nose Throat Disorders $\underline{6}, 12$.

Walker T, Macfariane T, Garry GM (2007): The epidemiology and chronobiology of epistaxis: an investigation of Scottish hospital admissions 1995-2004. Clin Otolarnygol 32, 361-65.

Walz G (2011): Organisationsformen der Notfallmedizin aus Sicht des Krankenhausmangement. Chirurg $\underline{82}, 342-347$.

Weiß C, Bauer A, Die medizinische Doktorarbeit - von der Themensuche bis zur Dissertation. 3. Auflage; Thieme Verlag, Stuttgart 2008.

Wheatley A, Temple R, Camilleri A, Jones P (1999): ENT open access clinic: an audit of a new service. J Laryngol Otol 113, 657-660.

Windfuhr JP, Remmert S (2005): Peritonsillarbszess. HNO $\underline{53}, 46-57$.

Windfuhr JP, Sesterhenn K (2001): Blutung nach Tonsillektomie Analyse von 229 Fällen. HNO $\underline{49}, 706-712$. 


\section{Danksagung}

Mein besonderer Dank gilt Herrn Professor Laskawi aus der Klinik für Hals-Nasen-Ohrenheilkunde im Zentrum Augenheilkunde und Hals-, Nasen-, Ohrentheilkunde der Medizinischen Fakultät der Universität Göttingen für die Überlassung des interessanten Themas und dessen Betreuung. Weiterhin möchte ich Herrn Professor Bauer für seinen wertvollen wissenschaftlichen Rat danken. Frau Bruhse möchte ich ebenfalls für die freundliche Unterstützung während meiner Datenerhebung danken. Herrn Dr. Simon Schneider aus dem Institut für Medizinische Statistik der Universität Göttingen danke ich für die Hilfe bei der statistischen Auswertung. 